FUEL REGRESSION RATE ESTIMATION MODEL FOR SWIRLING-FLOW HYBRID ROCKET ENGINES

\author{
By \\ Potchara Wongyai, B.Eng \\ Mechanical Engineering \\ Memorial University of Newfoundland, 2013
}

A thesis presented to Ryerson University

in partial fulfillment of the

requirements for the degree of

Master of Applied Science

In the Program of Aerospace Engineering

Toronto, Ontario, Canada, 2015

OPotchara Wongyai 2015 


\section{Author's Declaration for Electronic Submission of a Thesis}

I hereby declare that I am the sole author of this thesis. This is a true copy of the thesis, including any required final revisions, as accepted by my examiners.

I authorize Ryerson University to lend this thesis to other institutions or individuals for the purpose of scholarly research.

I further authorize Ryerson University to reproduce this thesis by photocopying or by other means, in total or in part, at the request of other institutions or individuals for the purpose of scholarly research.

I understand that my thesis may be made electronically available to the public. 


\title{
FUEL REGRESSION RATE ESTIMATION MODEL \\ FOR SWIRLING-FLOW HYBRID ROCKET ENGINES
}

\author{
Potchara Wongyai \\ Master of Applied Science, Aerospace Engineering, Ryerson University
}

(2015)

\begin{abstract}
In the current study, an analytical model to estimate the fuel surface regression rate of hybrid rocket engines with head-end swirling flow oxidizer injection is established. The model is based on a convective heat feedback approach and, in conjunction with the corresponding boundary layer (or zone) concept which accounts for transpiration, effective hydraulic diameters, and wall friction. The effective tangential (swirl) velocity of the gas provides a positive augmentation effect to the fuel regression rate, above that due to the axial mass flux component of the core gas flow. From the literature, a variety of propellant combinations, engine sizes, and flow swirl numbers are evaluated for engines having circular-port fuel grains, with sample results provided in this report for comparative purposes. The predicted fuel regression rates for the most part compare quite well with the corresponding experimental data. Additionally, the validity of the underlying assumption of a slowly decaying effective axial and tangential velocity of the gas as one moves downstream along the central fuel port is to some degree verified using a computational approach, based on a simplified engine flow model. As a final element of the overall study, the fuel regression rate model is evaluated for parameter sensitivity. The settings for some propellant and gas properties are found to have a significant influence on the quantitative predictive results.
\end{abstract}




\section{Acknowledgements}

This thesis report on swirl effects in hybrid rocket engines originates in part from the fundamental theory and knowledge established in the Ryerson University study of fuel regression rates in a non-swirling axial flow HRE, earlier work which was carried out by my supervisor, Professor David Greatrix. Great advice and suggestions for the present follow-on research study have been given by Prof. Greatrix throughout my Master's program at Ryerson University. Therefore, I would like to thank my supervisor, who supported me along the journey of completing this effort and inspired me as a role model for conducting rocket research.

I would also like to thank all friends and co-workers who gave ideas and suggestions regarding this research topic, as well as helping me in finding necessary information and document from various sources. Specially, I would like to give an acknowledgement to Shivanand Patil, my coworker and a senior research student, who guided me though the initial phase of the research, especially in understanding fundamental theories and the usage of CFD software.

Lastly and most importantly, I would like to thank my parents who provided me with great suggestions and wisdoms regarding the research study, altogether with financial supports during the period of my study.

All the success and accomplishment from this research study would not have been available without the people mentioned above; thus, I would like to sincerely thank everyone again for their parts of the contribution and their kindness. 


\section{Table of Contents}

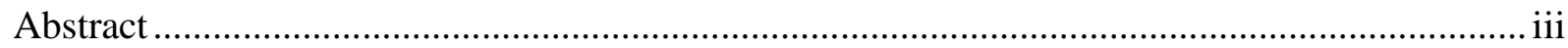

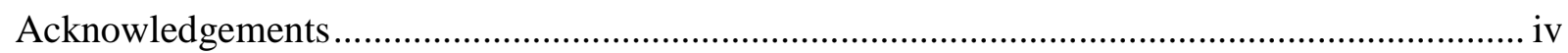

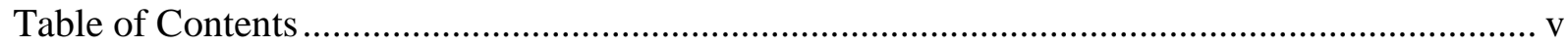

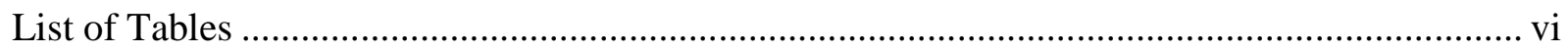

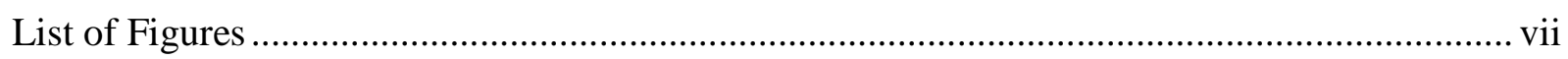

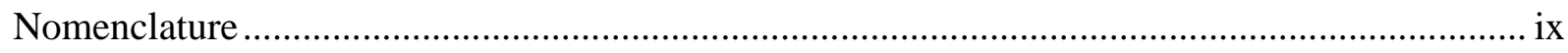

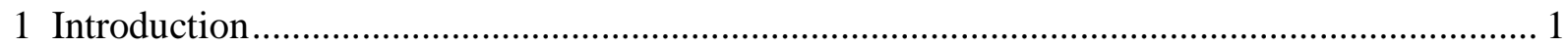

2 Model Background and Previous Development ….................................................................. 4

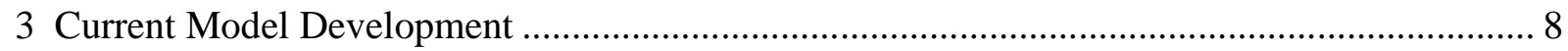

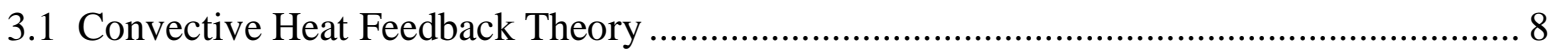

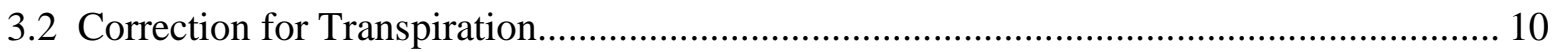

3.3 Calculation of Heat Transfer Coefficients and Friction Factors ..................................... 14

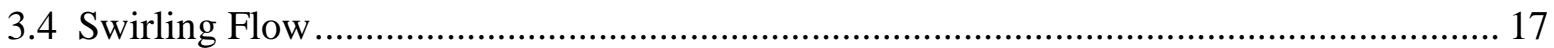

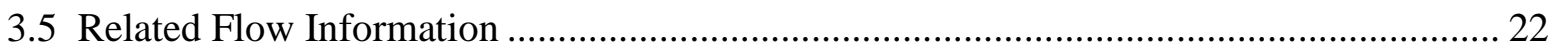

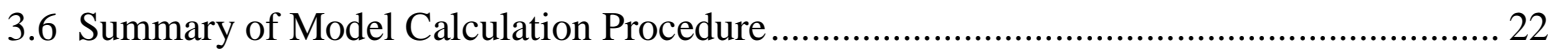

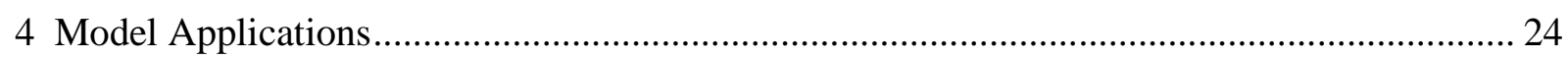

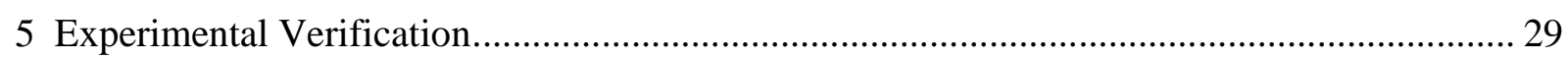

6 Computational Fluid Dynamic Analysis of a Simplified Engine Flow Model........................ 35

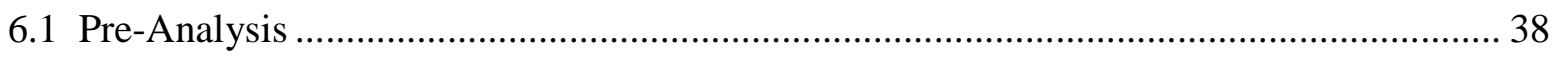

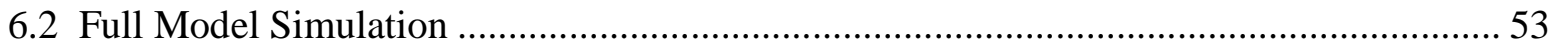

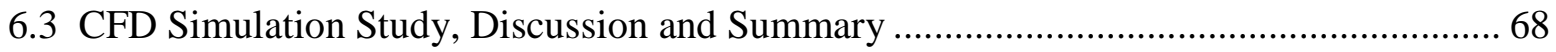

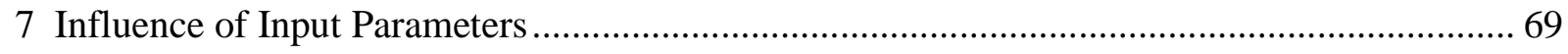

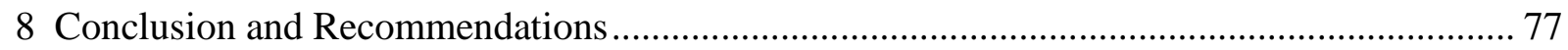

\section{List of Appendices}

Appendix A: Sample Results from Quasi-Steady Analysis...................................................... 79

Appendix B: Mini-Program for Input Parameter Study ............................................................. 81

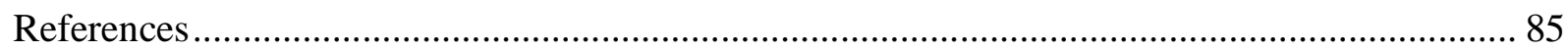




\section{List of Tables}

Table 1: Engine Characteristics of Experimental Hybrid Rocket Engines ................................. 30

Table 2: Propellant Gas and Initial Properties ........................................................................ 37

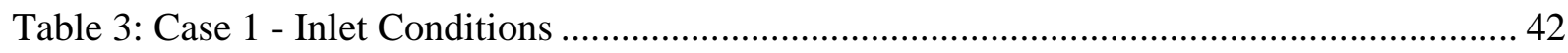

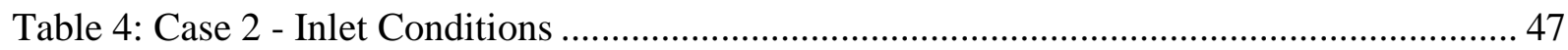

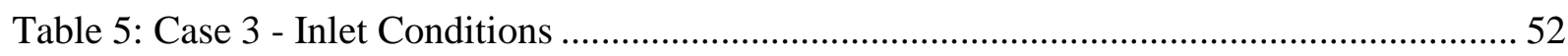

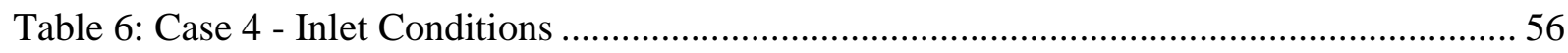

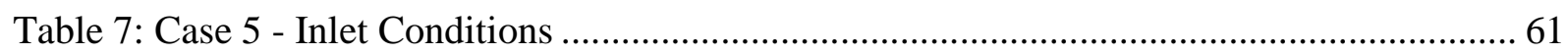

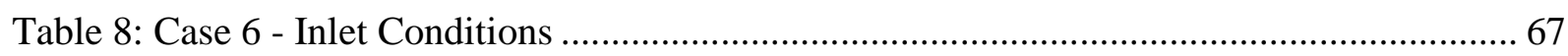

Table 9: Percentage Increase of Regression Rate by Each Parameter (at $\mathrm{G}=500 \mathrm{~kg} / \mathrm{s}-\mathrm{m}^{2}$ )....... 70 


\section{List of Figures}

Figure 1: Conventional Hybrid Rocket Engine with Head-End Oxidizer Injection. 2

Figure 2: Predictive Model Results and Corresponding Experimental Data, for Fuel Regression Rate as Function of Axial Mass Flux, using Same HTPB/GOX Propellant at Different Engine Port Diameters ${ }^{10}$

Figure 3: Predictive Model Results and Corresponding Experimental Data, for Fuel Regression Rate as Function of Axial Mass Flux, for Differing Propellants and Engine Sizes ${ }^{10}$................... 7

Figure 4: Cross-Section of Port at Head-end and Mid-Body of the Fuel Grain .......................... 16

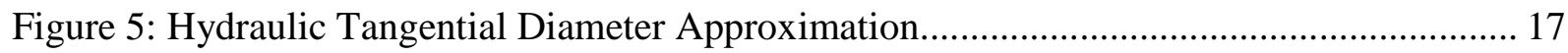

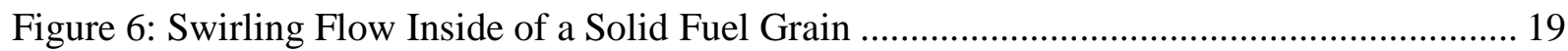

Figure 7: Swirl Number Comparison between Plug Flow and Forced-Vortex Flow ................... 19

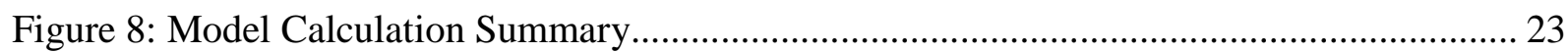

Figure 9: Predictive Model Results and Corresponding Experimental Data, ${ }^{4}$ for Fuel Regression

Rate as Function of Mass Flux and Swirl Number, for Same Paraffin/GOx Propellant .............. 31

Figure 10: Predictive Model Results and Corresponding Experimental Data, ${ }^{5}$ for Fuel Regression

Rate as Function of Mass Flux and Swirl Number, for Same HDPE/ $\mathrm{N}_{2} \mathrm{O}$ Propellant ................ 32

Figure 11: Predictive Model Results and Corresponding Experimental Data, ${ }^{6}$ for Fuel Regression

Rate as Function of Mass Flux and Swirl Number, for Same HTPB/GOx Propellant ................. 33

Figure 12: CFD Model for Hybrid Rocket Engine (Dimensions in Meters) ............................... 36

Figure 13: Case 1 - Streamline, Pressure, and Axial Velocity Profiles for Engine ....................... 40

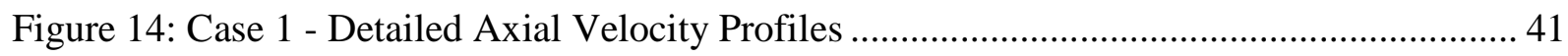

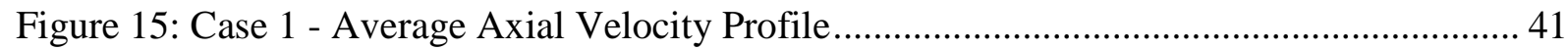

Figure 16: Case 1 - Detailed Pressure Profiles ........................................................................... 42

Figure 17: Case 2 - Streamlines, Pressure, Axial Velocity, and Tangential Velocity .................. 44

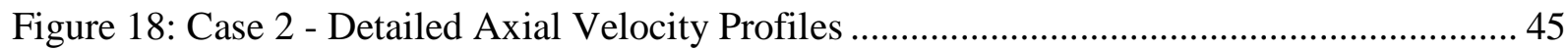

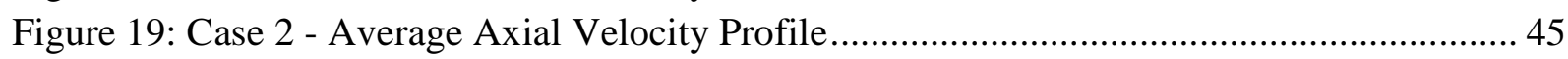

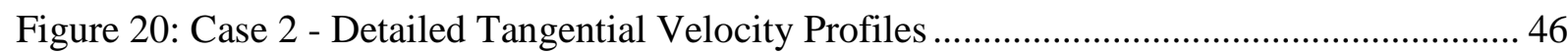

Figure 21: Case 2 - Average Tangential Velocity Profile............................................................ 46

Figure 22: Case 2 - Detailed Pressure Profiles ........................................................................... 47

Figure 23: Case 3 - Streamlines, Pressure, Axial Velocity, and Tangential Velocity ................. 49

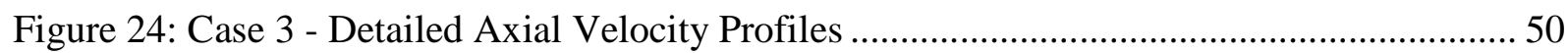

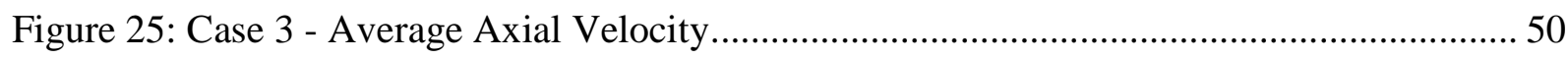

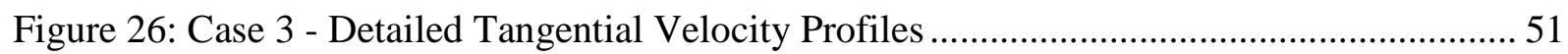

Figure 27: Case 3 - Average Tangential Velocity ....................................................................... 51

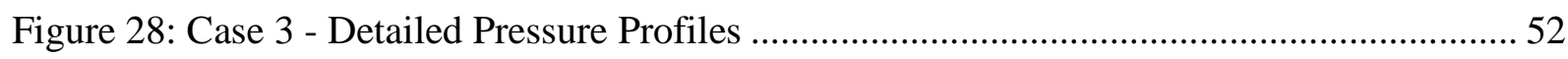

Figure 29: Case 4 - Streamlines, Pressure, and Axial Velocity .................................................... 54

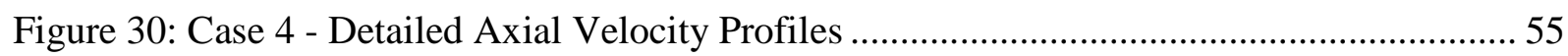

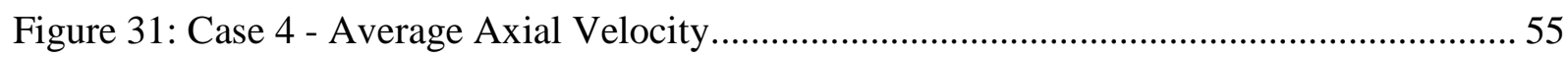

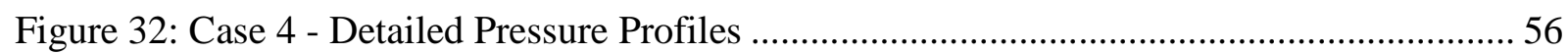

Figure 33: Case 5 - Streamlines, Pressure, Axial Velocity, and Tangential Velocity ................... 58 


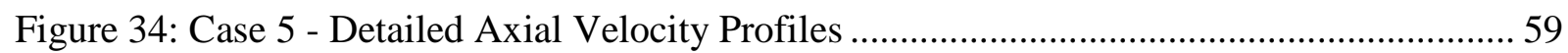

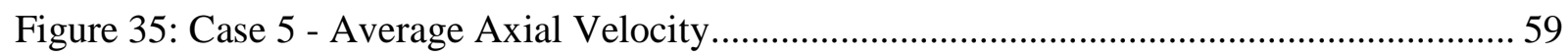

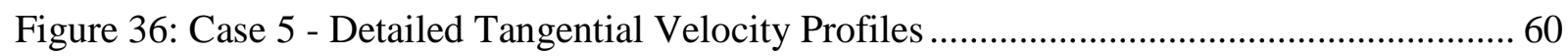

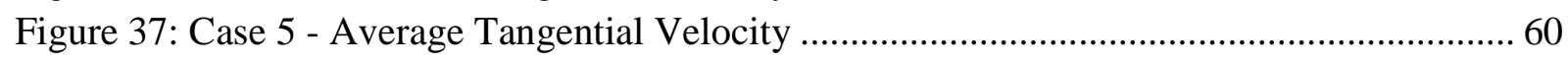

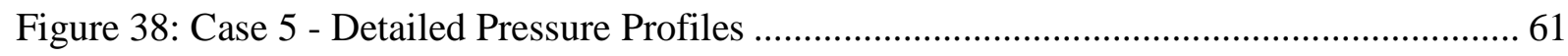

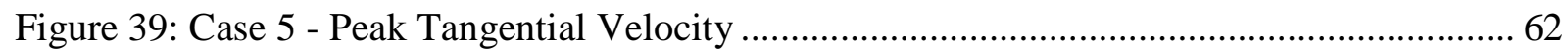

Figure 40: Case 6 - Streamlines, Pressure, Axial Velocity, and Tangential Velocity .................. 64

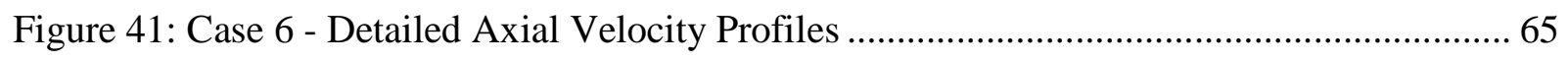

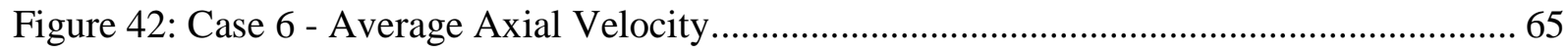

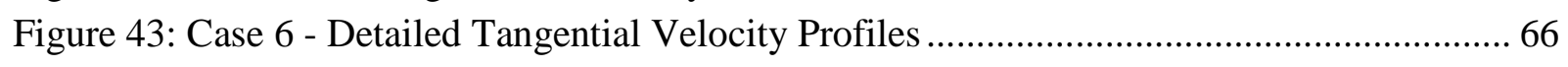

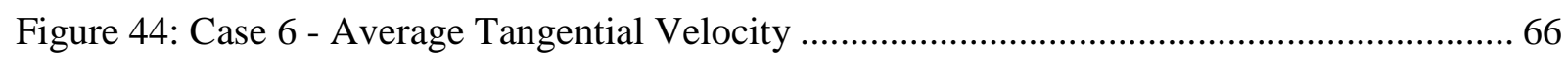

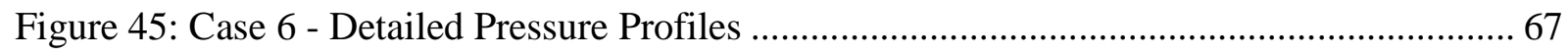

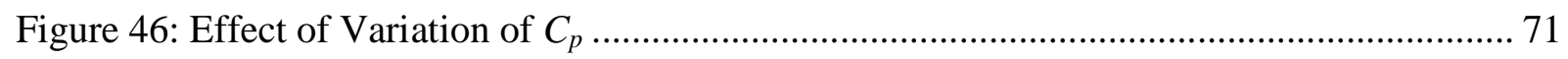

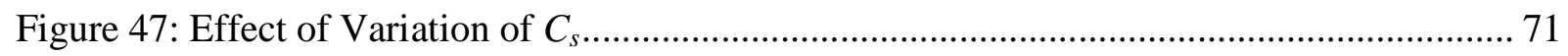

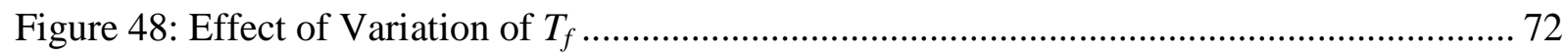

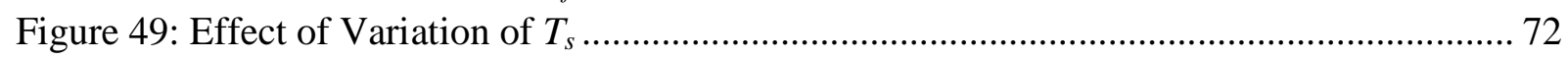

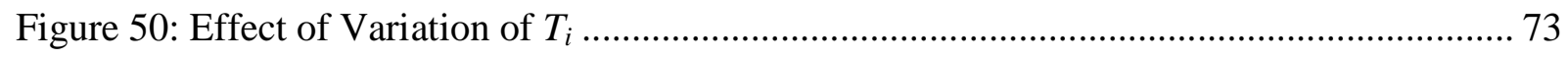

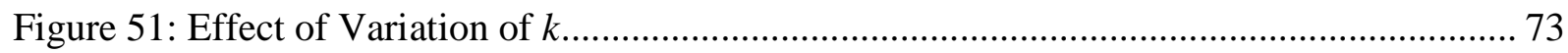

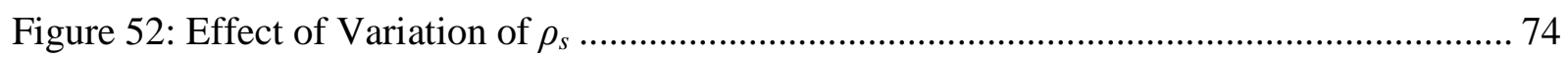

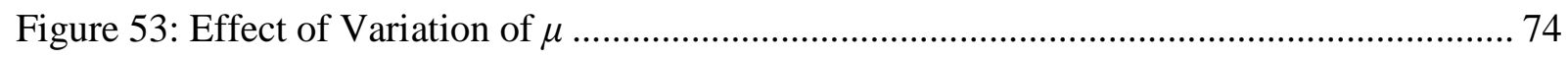

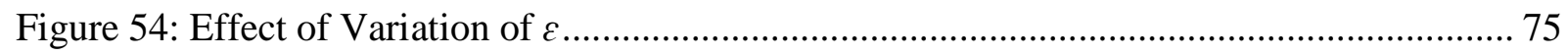

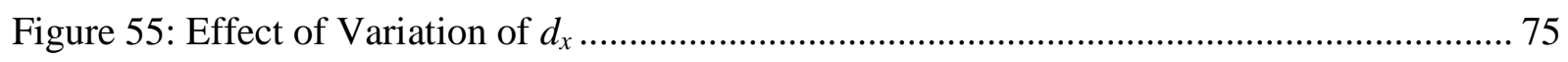

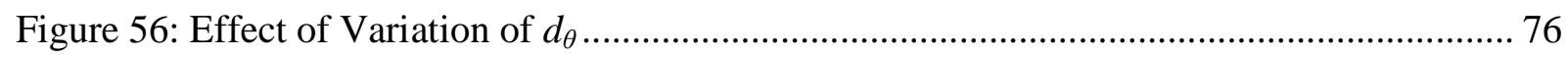

Figure 57: Head-End Pressure Prediction by QSHYB of a Swirl Flow $(S=2$, at $3 \mathrm{~s}<t<10 \mathrm{~s})$.

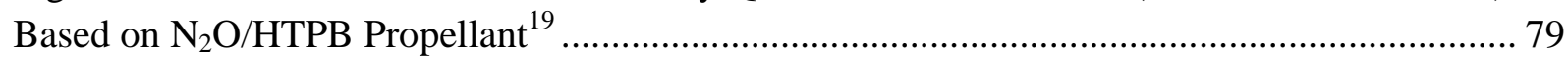

Figure 58: Thrust Prediction by QSHYB of a Swirl Flow $(S=2$, at $3 \mathrm{~s}<t<10 \mathrm{~s})$. Based on

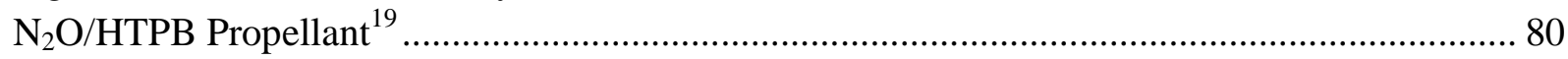

Figure 59: Stoichiometric Length Prediction by QSHYB of a Swirl Flow $(S=2$, at $3 \mathrm{~s}<t<10 \mathrm{~s})$.

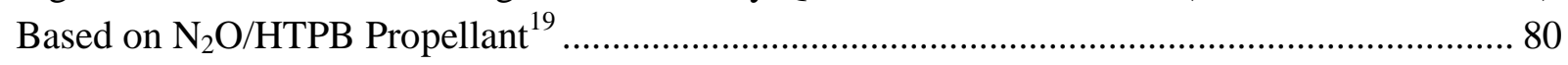

Figure 60: User Interface for Input-Parameter Study Program ............................................. 81 


\section{Nomenclature}

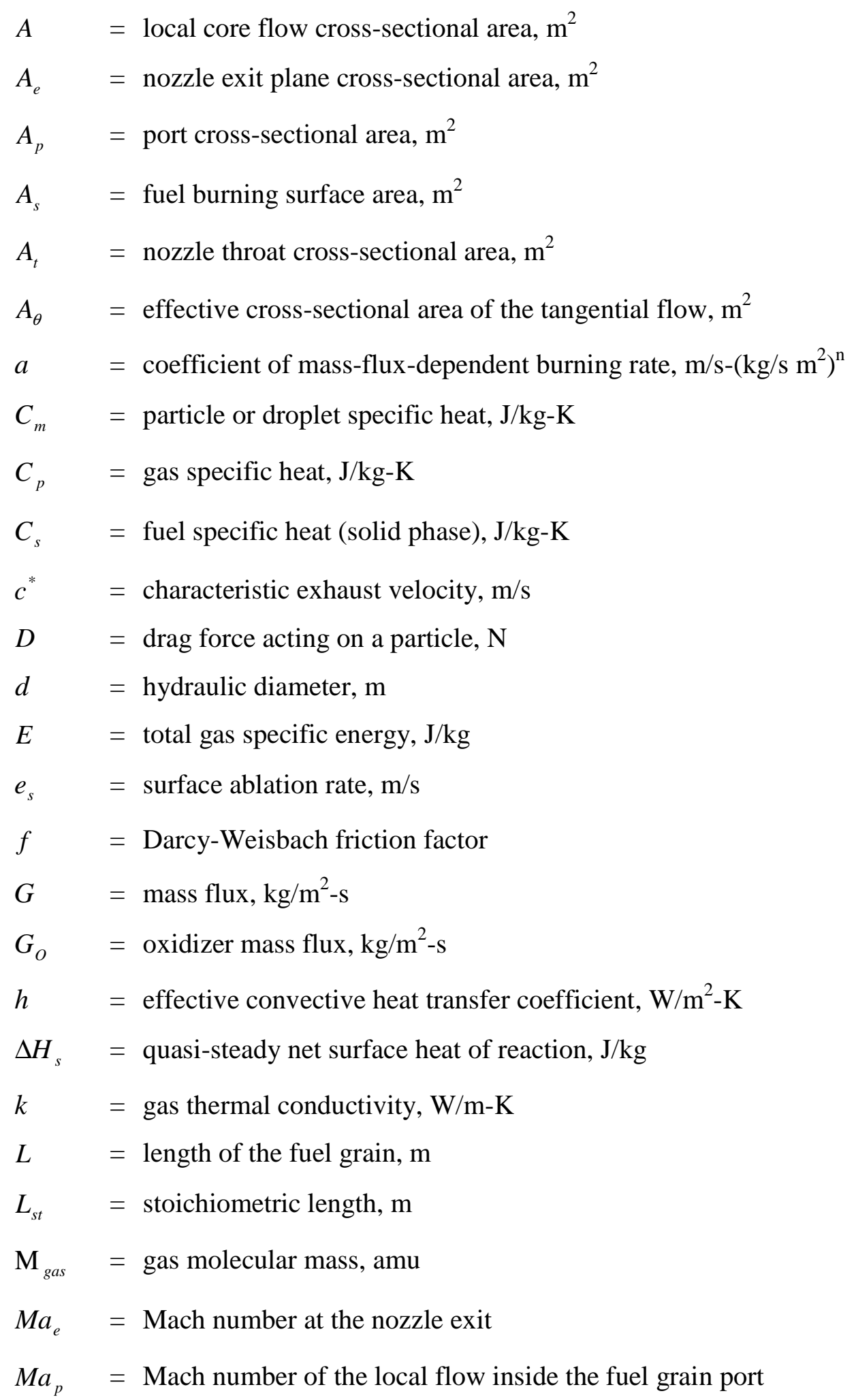




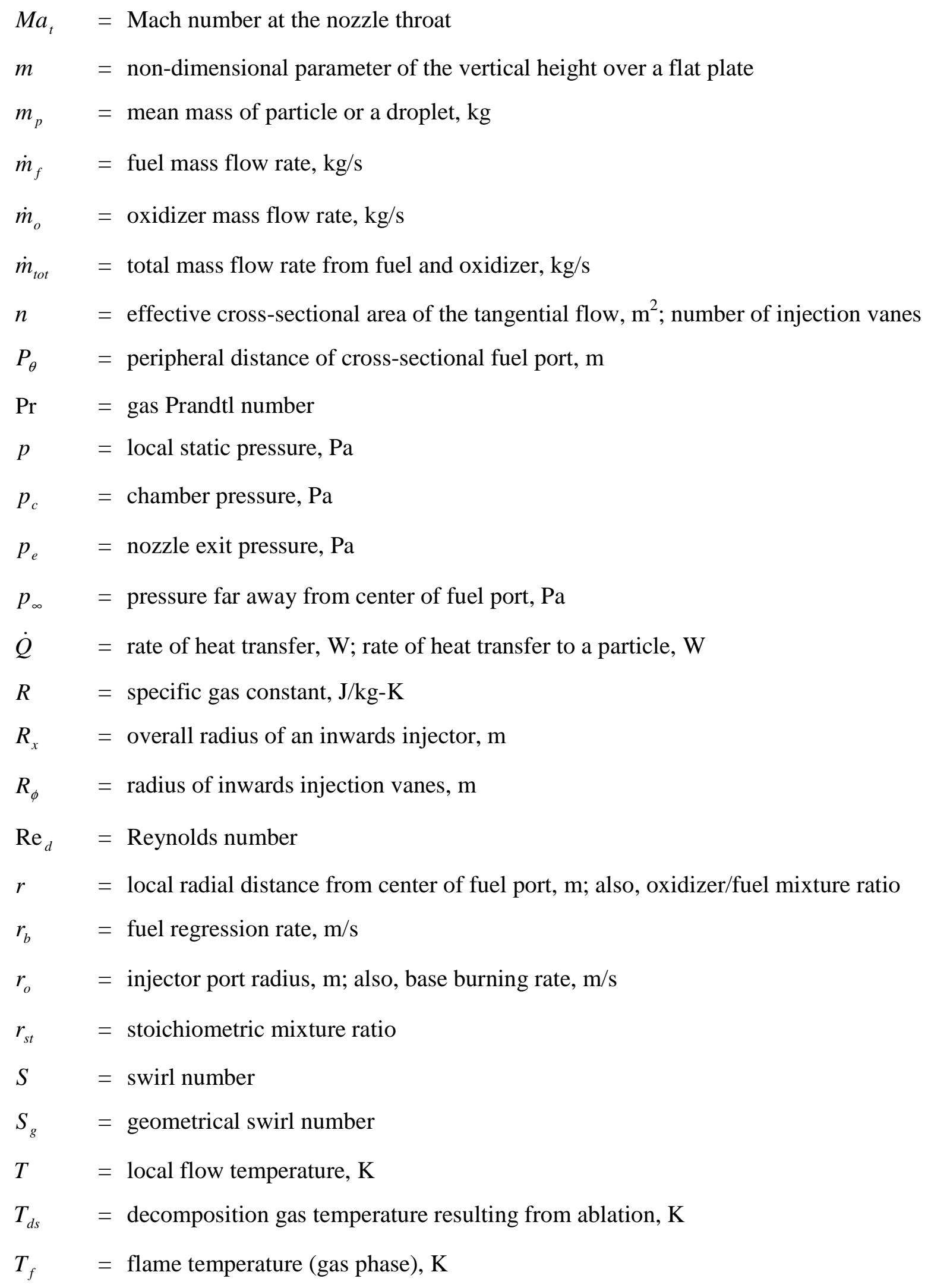




$$
\begin{array}{ll}
T_{i} & =\text { fuel initial temperature (solid phase), } \mathrm{K} \\
T_{s} & =\text { fuel burning surface temperature, } \mathrm{K} \\
T_{\infty} & =\text { local central core gas temperature, } \mathrm{K} \\
U & =\text { effective (bulk) axial velocity, } \mathrm{m} / \mathrm{s} \\
u & =\text { local axial velocity, } \mathrm{m} / \mathrm{s} \\
V & =\text { effective tangential velocity, } \mathrm{m} / \mathrm{s} \\
v & =\text { local tangential velocity, } \mathrm{m} / \mathrm{s} \\
w & =\text { vertical velocity over a flat plate, } \mathrm{m} / \mathrm{s} ; \text { normal gas velocity of flame, } \mathrm{m} / \mathrm{s} \\
x & =\text { longitudinal distance along the fuel grain port, } \mathrm{m} \\
y & =\text { vertical height over a flat plate, } \mathrm{m} \\
\alpha & =\text { particle mass loading fraction } \\
\beta & =\text { non-dimensional parameter for temperature ratio } \\
\Delta & =\text { film thickness from film theory, } \mathrm{m} \\
\delta & =\text { effective separation distance from swirl injector port's exit to fuel surface, } \mathrm{m} \\
\varepsilon & =\text { fuel surface roughness height, } \mathrm{m} \\
\gamma & =\text { gas ratio of specific heats } \\
\mu & =\text { gas absolute viscosity, } \mathrm{kg} / \mathrm{m}-\mathrm{s} \\
\rho & =\text { gas density, } \mathrm{kg} / \mathrm{m}^{3} \\
\rho_{s} & =\text { fuel density (solid phase) }, \mathrm{kg} / \mathrm{m}^{3} \\
\Gamma & =\text { non-dimensional parameter } \\
& =\text { swirl angle of oxidizer injector, deg } \\
& =
\end{array}
$$

\section{Subscripts and Superscripts}

$$
\begin{array}{ll}
p & =\text { subscript for particulate parameters } \\
x & =\text { subscript for axial direction } \\
\theta & =\text { subscript for tangential direction } \\
* & =\text { superscript indicating zero-transpiration case } \\
\text { comp } & =\text { subscript for compressible flow regime } \\
\text { incomp } & =\text { subscript for incompressible flow regime }
\end{array}
$$




\section{Introduction}

Hybrid rocket engines (HREs) have several performance characteristics that are potentially favorable to a variety of rocket operations. They have more nominal advantages than solid and liquid rocket engines in some flight applications, owing in part to the difference between the propellant phases, where the oxidizer is kept in a liquid/ gaseous phase and fuel is kept in a solid phase, allowing them to be safely separated and controlled easily. A schematic diagram of a conventional HRE is shown in Fig. 1. Hybrid rocket engines' positive features, such as the aforementioned enhanced safety when they are being operated, also include an ability to control thrust, and a nominal capability for shutting down and restarting, while in flight. Moreover, they are commonly quite simple to be manufactured and are typically less expensive, relatively speaking. Nevertheless, there are some design concerns surrounding hybrid rocket engines. For example, everything else being equal, thrust provided by conventional HREs is commonly lower compared to other types of chemical rocket engines. Other concerns for hybrid rocket engines include: slow fuel regression rates, low volumetric loading, unburnt fuel residuals, shifting of the mixture ratio, and mixing/combustion efficiencies. ${ }^{1}$ The improvement of HREs is of interest for a number of institutions and commercial firms around the world, where the goal is to minimize the problems caused by the issues listed above.

As one of the major problems related to HREs, slow fuel surface regression rates are being dealt with using various design techniques. One should note that larger engines (i.e., with larger port diameters) have even slower fuel regression rates, to potentially further exacerbate this issue. Certain fuels, such as paraffin wax, have a considerably higher regression rate relative to conventional fuels like polyethylene (plastic) and polybutadiene (rubber). However, paraffin wax has drawbacks, such as its inherent structural softness relative to the more rigid plastics and rubbers.

If one is restricted to using a conventional fuel, a common technique for increasing the overall propellant mass flow is to increase the burning fuel surface area (hence compensating for the lower fuel regression rate). One can replace a conventional cylindrical fuel grain (with a single 
central port) with a more complicated geometry that has a greater internal surface area. In this regard, some of the common designs include star-port, wagon-wheel-port, multi-port, and helical-port fuel grain configurations. However, bear in mind that a single-port cylindrical grain configuration is the most desirable choice for a variety of reasons. If one is striving to retain a cylindrical-grain configuration and still use a conventional fuel, a promising technique for regression rate augmentation is to effectively increase the velocity of the gas flow over the fuel surface. The implementation of this technique can be done by utilizing a swirl flow, which would positively augment the baseline axial-flow burning mechanism.

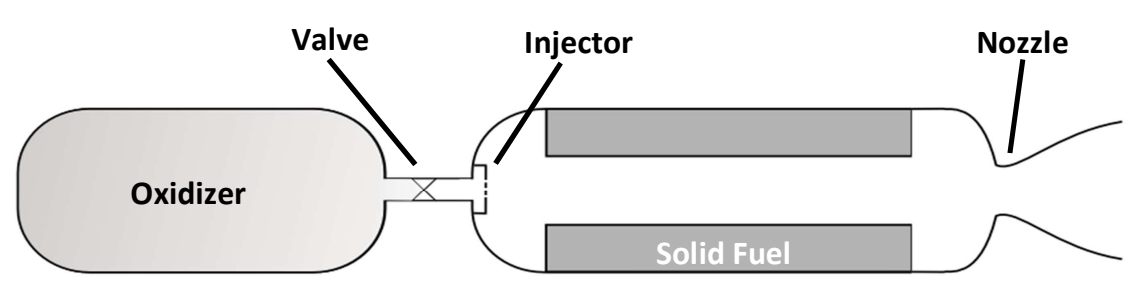

Figure 1: Conventional Hybrid Rocket Engine with Head-End Oxidizer Injection

The implementation of oxidizer injection with a swirl flow has been studied experimentally and theoretically by a number of institutions and companies. These studies illustrated that one could obtain substantial increases of fuel regression rates by increasing the swirl strength of the central gas flow. One notable example in this regard were experiments conducted by ORBITEC $^{\mathrm{TM}}$, where the test engine was using a forward- and aft-injection, reversing-vortex-flow approach. The experimentally measured fuel regression rate was observed to increase by as much as 8 times compared to the standard non-swirling flow configuration. ${ }^{2}$ Similarly, albeit with different swirl injection configurations, other experimental studies of HREs have also clearly shown a positive augmentation of the fuel regression rate due to swirl. ${ }^{3-6}$

A swirl flow provides an advantage over a standard axial flow since it adds an additional tangential velocity component to the gas, allowing the flow to achieve a higher absolute velocity 
for the same mass flow rate of the oxidizer. Given the nature of diffusion flame behavior in HREs, the mass-flux-dependent fuel regression rate will increase accordingly. In some cases, as with head-end oxidizer injection, a swirl flow also helps improve the flame-holding stability due to the recirculation zone caused by a swirling gas flow. ${ }^{5}$

There is an ongoing effort at Ryerson University to study various means for better HRE performance prediction, in conjunction with efforts to investigate various ways for improving HRE performance. Swirling oxidizer injection from the engine's head end certainly provides a straightforward means for enhancing conventional HRE performance, potentially allowing for the use of a single-port cylindrical grain design for larger engines. To date, models for prediction of swirl augmentation of fuel burning rate have been quite limited in their utility and accuracy. The objective of the present study is to develop a predictive model for predicting the fuel regression rate with some accuracy and more general utility, for a conventional head-end injection engine that may be using swirl. Other issues related to swirl flow in HREs will also be looked at in this thesis.

For the content arrangement, the research background and previous development of the baseline predictive model (non-swirling axial flow) are presented in Chapter 2, followed by the development of the present model (swirling flow) in Chapter 3, which is the main contribution of this research. Chapter 4 shows applications of the model in the internal ballistic analysis for HREs. For a verification of the model, comparisons of experimental and predictive results are presented in Chapter 5. Additionally, a CFD study of a simplified engine is carried out to better understand some characteristics of a swirl flow, as explained in Chapter 6. Lastly, input parameters of the model are further investigated for their sensitivity effects on the calculated values of regression rate, as provided in Chapter 7. 


\section{Model Background and Previous Development}

A distinctive characteristic of a conventional hybrid rocket engine is the fuel grain's surface regression rate being predominantly a function of the effective mass flux ( $G=\rho u_{x}$, for axial gas flow) moving above the fuel surface. Knowing the level of regression rate is important for finding the mass flow rate of fuel $\dot{m}_{f}$ being removed from the fuel grain, which in turn establishes the effective mixture ratio $\dot{m}_{O} / \dot{m}_{f}$ in combining with the oxidizer flow $\dot{m}_{O}$ originating from the head-end injector(s). The combined mass flow between fuel and oxidizer becomes the total gaseous mass flow which continuously increases as more fuel is being added, predominantly via combustion, along the length of the fuel grain as one moves downstream. Conventionally, the combustion-based regression rate $r_{b}$ at a local point on the fuel grain can be evaluated based on the total axial mass flux $G$ of the central gas flow, using the empirical factors, $a$ and $n$, obtained from various experimental test firings. The general empirical equation for regression rate in hybrid rocket engines is shown in Eq. 2.1 ${ }^{7}$ :

$$
r_{b}=a G^{n}
$$

Note that the value of coefficient $a$ changes inversely with respect to the size of the fuel grain port diameter ( $a$ gets lower as $d_{p}$ increases), and exponent $n$ ranges between 0.4 to 0.85 in a typical engine. ${ }^{7}$ Since the total mass flow also relies on the amount of fuel being removed (in transforming from a solid to a gas, via combustion), several iterations may be required for a proper evaluation of fuel regression rates. An approximation of regression rates can also be done by assuming that the effective mass flux is relatively constant; therefore, the original relationship between regression rate and total local mass flux can be approximated using the incoming mass flow rate of the oxidizer, as shown in Eq. $2 \cdot 2^{7}$ :

$$
r_{b} \approx a G_{O}^{n}
$$

Conducting an engine firing test generally takes time and can be expensive. Therefore, this is not a desirable option early on in the design process, especially during a preliminary design stage 
where variations of the rocket engine's performance need to be investigated. A mathematical model that can predict engine performance (ideally without empirical factors, such as used for Eqs. 2.1 and 2.2) to a certain degree of accuracy is desirable. To date, a number of comprehensive predictive models for fuel surface regression rate have been proposed. ${ }^{1,8,9}$ A common issue with these models is the lack of universality when being applied subsequently to other engines, engines that differ to some degree from the reference engine used for the given model's original development.

The model to be presented in this study is constructed using convective heat feedback theory, a common approach used by past HRE researchers, given the clear relationship between axial mass flux $G$ of the central gas flow and the resulting observed burning rate of the fuel surface. This approach has been used successfully in previous Ryerson University (RU) investigations of solid-propellant rocket motor erosive burning, as well as more recent investigations of nonswirling axial-flow-dependent combustion in HREs. An RU effort produced an effective predictive model of fuel regression rate for HREs employing conventional non-swirling axialflow head-end oxidizer injection. The RU model was constructed based on a one-dimensional scheme, where the effects of transpiration (mass transfer from the fuel surface, a.k.a., "blowing"), port diameter and wall friction were incorporated. The model's principal equation for regression rate, when no other regression rate mechanism is present (e.g., radiation, pressure, normal acceleration), is shown below ${ }^{10}$ :

$$
r_{b}=\frac{h_{x}^{*}}{\rho_{s} C_{p}} \ln \left[1+\frac{C_{p}}{C_{s}} \frac{\left(T_{f}-T_{s}\right)}{\left(T_{s}-T_{i}-\Delta H_{s} / C_{s}\right)}\right]
$$

Note that $h_{x}^{*}$ is a non-transpiration effective heat transfer coefficient calculated from the effective axial velocity (and corresponding axial mass flux) of the core gas. Further explanation and theoretical derivation of the model (there are a number of supporting equations in addition to Eq. 2.3), which acts as a framework for the new swirl model content that is to be included, are provided in Chapter 3 of this thesis. 
To verify the accuracy of the model used in the originating RU study, predictive results of regression rates were compared with experimental results obtained from various experimental studies reported in the literature. Each of the experiments done at other institutions used a hybrid rocket engine with a cylindrical fuel grain, and nominally non-swirling oxidizer injector(s) located at the head end of the engine. The overall setup configurations were similar to the diagram shown in Fig. 1. The RU model's results compared quite well to the experimental results for various oxidizer and fuel combinations, as shown in Figs. 2 and 3. Although each engine in this evaluation differed in size and in other respects, the model could still predict the results quite accurately for non-swirling axial flow; therefore, the motor scale effect, which dictates a reduction in the fuel regression rate as the port diameter becomes larger, was accounted for by this predictive model.

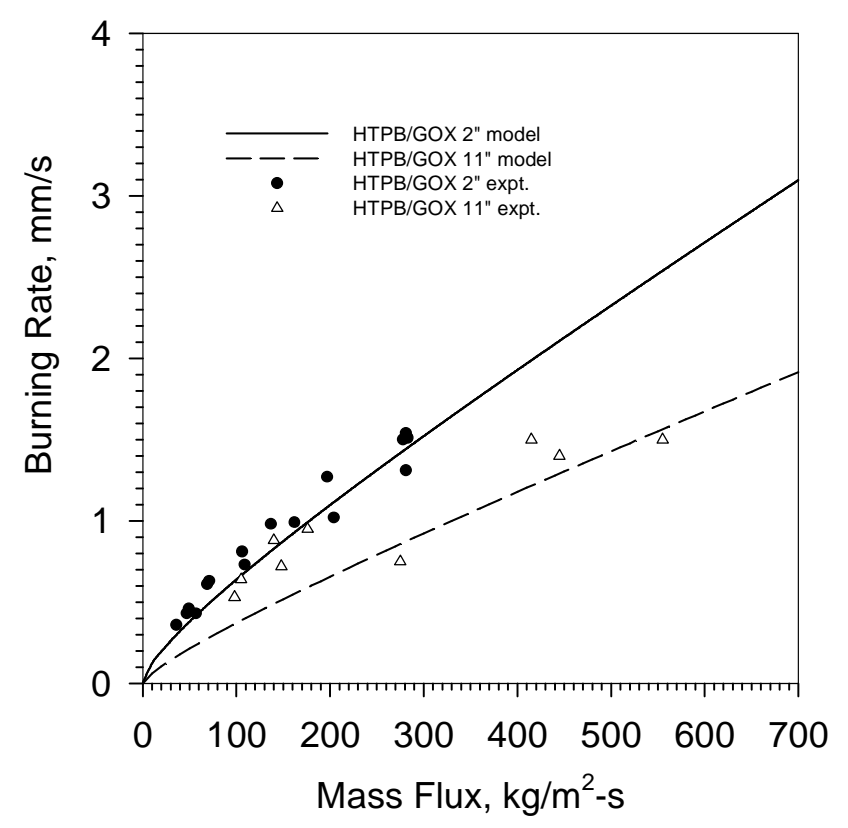

Figure 2: Predictive Model Results and Corresponding Experimental Data, for Fuel Regression Rate as Function of Axial Mass Flux, using Same HTPB/GOX Propellant at Different Engine Port Diameters $^{10}$ 


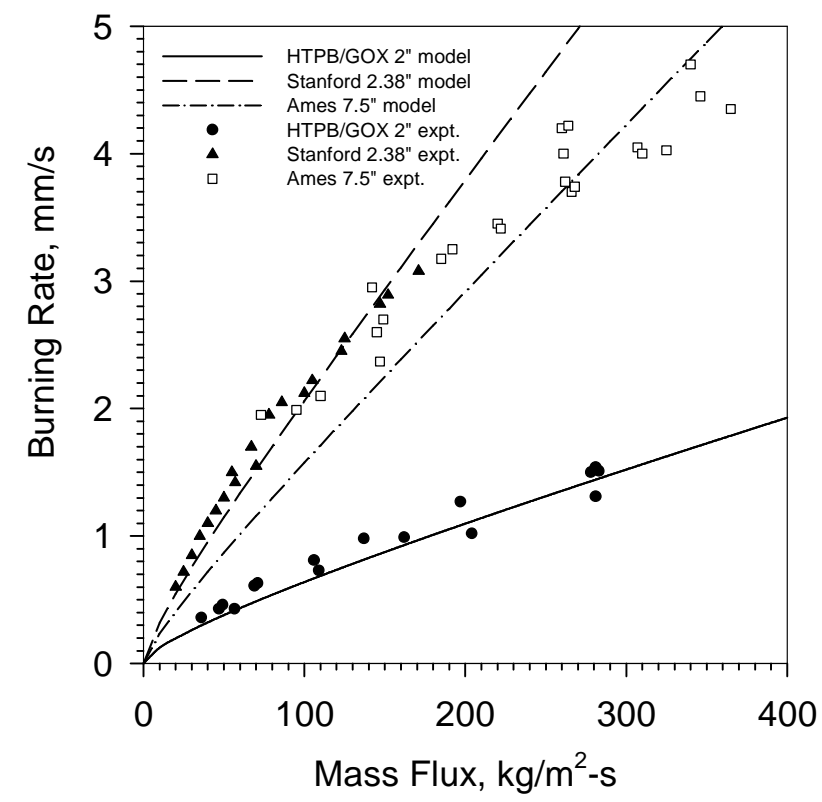

Figure 3: Predictive Model Results and Corresponding Experimental Data, for Fuel Regression Rate as Function of Axial Mass Flux, for Differing Propellants and Engine Sizes ${ }^{10}$ 


\section{Current Model Development}

The present regression rate model ${ }^{11}$, a principal contribution of this thesis, is an extension of the axial-flow regression rate model which was developed in the previous RU study, ${ }^{10}$ to now allow for swirling flow as evolving from a head-end oxidizer injector. This one-dimensional model is constructed based on convective heat feedback theory, ${ }^{12}$ with the incorporation of the effects of transpiration, hydraulic diameters and wall friction. The model includes the contribution of a swirl flow, where a positive augmentation due to the swirling gas flow is applied to the fuel regression rate. An eligible hybrid rocket engine for using this model is one having a solid fuel grain that is cylindrical in shape, and the oxidizer being injected in a swirl pattern from the head end of the engine. Model explanations and corresponding theoretical derivations for the updated regression rate model will be provided in this chapter. The overview of the calculation process from start to end, as a further aid to the reader, is provided in Section 3.6.

\subsection{Convective Heat Feedback Theory}

Convective heat feedback theory, through energy conservation principles, allows one to utilize the relationship between the energy entering the burning fuel surface from the gas phase (the convective heat transfer component) and the heat energy leaving (solid-phase heat energy removal via mass transfer). The convective heat transfer is driven by the difference between the effective core flow flame temperature $T_{f}$ and the burning fuel surface temperature $T_{s}$. The heat energy of solid fuel mass evolving (ultimately as a gas) from the burning surface is governed by the change of the solid fuel temperature starting from its initial temperature $T_{i}$ to its current temperature at the burning surface $T_{s}$. To better account for all the heat transfer effects that one might encounter in practice, the net surface heat of reaction $\Delta H_{s}$ can also be incorporated into the model, if it is appreciable. This quantity is a heat transfer contribution due to either an endothermic process nominally at the fuel burning surface, when the net surface heat of reaction is negative (absorbing heat), or an exothermic process, when the net surface heat of reaction is positive (releasing heat). From past studies for quasi-steady burning in HREs, the surface heat of reaction has had little effect on the overall heat transfer for the type of fuels being considered; therefore, it is commonly neglected $\left(\Delta H_{s}=0\right)$ in the calculations being done. ${ }^{10-12}$ Additionally, 
convective heat transfer for the general type of propellant being used in this study is the main mechanism for the overall heat transfer process, while other heat transfer mechanisms, such as radiation, are assumed to have a much less significant contribution; therefore, these secondary mechanisms are excluded for the initial analysis. In cases when other heat transfer mechanisms may in practice become more significant, an additional component contributing to regression rate may be included, as explained later in this section.

For the convective heat feedback theory, the governing equations for both of the heat transfer inputs, from the gas phase and solid phase, are shown below:

$$
\begin{gathered}
\dot{Q}=h_{e f f} A_{s}\left(T_{f}-T_{s}\right) \\
\dot{Q}=\dot{m}_{f}\left[C_{s}\left(T_{s}-T_{i}\right)-\Delta H_{s}\right]
\end{gathered}
$$

The effective convective heat transfer coefficient $h_{\text {eff }}$ accounts for both axial and tangential gas flow components, where heat transfer due to the tangential flow is considered to augment the baseline heat transfer resulted from the axial flow. The effective convective heat transfer coefficient can then be expressed as:

$$
h_{\text {eff }}=h_{x}+h_{\theta}
$$

An instantaneous regression rate of the solid fuel grain due to the effective convective heat transfer can be related to the above mechanisms via fuel mass flow rate. The local fuel mass flow rate can be expressed by:

$$
r_{b}=\frac{\dot{m}_{f}}{\rho_{s} A_{s}}
$$

where $A_{s}$ is the local reference burning surface area. 
Combining Eqs. 3.1 to 3.4, the regression rate formula due to the effective convective heat transfer can be formulated as shown below:

$$
r_{b}=\frac{\left(h_{x}+h_{\theta}\right)\left(T_{f}-T_{s}\right)}{\rho_{s}\left[C_{s}\left(T_{s}-T_{i}\right)-\Delta H_{s}\right]}
$$

The regression rate expression shown above is derived based on the assumption that the only mechanism contributing in a significant manner to regression rate is convective heat transfer. Commonly, for conventional HREs, this is the case. Nevertheless, if other regression rate driving mechanisms do exist to some degree, such as due to chamber pressure, radiation, and normal acceleration (the $a_{n}$ influence can be as a result of spinning the engine about its longitudinal axis), one can allow for a more general modeling approach. The effect of these mechanisms can be accounted for by applying the base regression rate $r_{o}$, which needs to be calculated separately, and then incorporated into the following more general equation:

$$
r_{b}=r_{o}+\frac{\left(h_{x}+h_{\theta}\right)\left(T_{f}-T_{s}\right)}{\rho_{s}\left[C_{s}\left(T_{s}-T_{i}\right)-\Delta H_{s}\right]}
$$

\subsection{Correction for Transpiration}

During the combustion process, fuel on the surface transforms from its original solid phase and subsequently evolves into the central core flow as a gas. As a result of this mass transfer, the solid fuel surface will of course regress with time. The mass transfer process can be modeled as fuel mass, ultimately as a gas, being "blown" radially into the core flow. This is called blowing or transpiration. Transpiration reduces friction experienced by the core flow near the surface (wall) of the solid fuel grain, thereby causing a reduction in the heat transfer value relative to the non-transpired case. The correction equations for the transpiration effect can be derived based on a two-dimensional flow over a flat plate experiencing blowing or suction at its surface. In this regard, theoretical as well as experimental investigations were carried out by Mickley et al. ${ }^{13}$ The theoretical modeling approach used by Mickley is commonly referred to as film theory. 
Film theory describes in an approximate manner the flow characteristics within an idealized boundary zone along the wall where the transpiration takes place. The boundary zone, in this case, is considered to be a very thin film with a conceptual thickness $\Delta$ (not to be confused with the actual flow entrance boundary thickness $\delta$, which is discussed in the later section), nominally representative of the region of transition between the radial flow and the core flow. Since the region is considered relatively thin, the flow inside the region can be assumed to be laminar, allowing for further simplification of the flow profile, which is potentially useful for subsequent theoretical derivations.

Heat transfer is a key mechanism of interest in this regression rate study, and the effect of transpiration on the heat transfer process needs to be included. With this in mind, the energy equation can be looked at in more detail at this juncture. One can note that diffusion and absorption are neglected in the film theory approach being discussed here. ${ }^{13}$ The energy equation can be framed for the transpiration flow, through the film zone, where $y$ represents the direction perpendicular to the wall and $w$ represents the vertical gas velocity:

$$
\frac{d T}{d y} \rho_{s} w C_{P}=\frac{d}{d y}\left(k \frac{d T}{d y}\right)
$$

or alternatively,

$$
0=k \frac{d T^{2}}{d^{2} y}-\left(\rho_{s} w C_{P}\right) \frac{d T}{d y}
$$

The second-order ordinary differential equation provides a general solution for the temperature with respect to the vertical distance from the wall surface as shown below:

$$
T(y)=e^{\left(\frac{\rho_{s} w C_{P}}{k}\right) y}+\text { Constant }
$$

The derivation can be further simplified, based on the assumption that heat transfer is constant within the region of the film due to its relatively small thickness: 


$$
-k\left(\frac{d T}{d y}\right)=h_{e f f}\left(T_{0}-T_{1}\right)
$$

In following Mickley's approach (and notation), using a non-dimensional parameter $\beta$ to track the change of the temperature, the heat transfer relationship can be rewritten as ${ }^{13}$ :

$$
\frac{d \beta}{d y}=\frac{h_{e f f}}{k}
$$

where

$$
\beta=\frac{T_{0}-T}{T_{0}-T_{1}} \quad ; \quad 0 \leq \beta \leq 1
$$

The heat transfer relationship from Eq. 3.9 can be substituted into the non-dimensional temperature parameter $\beta$. By introducing another non-dimensional parameter for film thickness $m$, an equation for $\beta$ can be further manipulated ${ }^{13}$ :

$$
\beta=\frac{\left(e^{\left(\rho_{s} w C_{P} / k\right) y}\right)_{0}-e^{\left(\rho_{s} w C_{P} / k\right) y}}{\left(e^{\left(\rho_{s} w C_{P} / k\right) y}\right)_{0}-\left(e^{\left(\rho_{s} w C_{P} / k\right) y}\right)_{1}}=\frac{e^{\left(\rho_{s} w C_{P} / k\right) y}-1}{\left(e^{\left(\rho_{s} w C_{P} / k\right) y}\right)_{1}-1}=\frac{e^{\Gamma m}-1}{e^{\Gamma}-1}
$$

and

$$
\frac{d \beta}{d m}=\frac{\Gamma\left(e^{\Gamma m}\right)}{e^{\Gamma}-1} \quad, \quad\left(\frac{d \beta}{d m}\right)_{0}=\frac{\Gamma}{e^{\Gamma}-1}(\text { at wall })
$$

where

$$
\begin{gathered}
m=\frac{y}{\Delta} \quad ; \quad 0 \leq m \leq 1 \\
\Gamma=\left(\frac{\rho w C_{P}}{k}\right) \Delta
\end{gathered}
$$

The Mickley parameter $\Gamma$ represents mass transfer resulting from transpiration. Applying the limit of $\Gamma$ in going towards a zero value, a non-transpiration case can be considered. Further 
using this parameter, the temperature ratio and its derivatives with respect to the height ratio at the wall location can be evaluated for the non-transpiration case:

$$
\begin{gathered}
\lim _{\Gamma \rightarrow 0} \beta=\lim _{\Gamma \rightarrow 0} \frac{e^{\Gamma m}-1}{e^{\Gamma}-1}=\lim _{\Gamma \rightarrow 0} \frac{\left(e^{\Gamma m}-1\right)^{\prime}}{\left(e^{\Gamma}-1\right)^{\prime}}=\lim _{\Gamma \rightarrow 0} \frac{m e^{\Gamma m}}{e^{\Gamma}}=m \\
\lim _{\Gamma \rightarrow 0}\left(\frac{d \beta}{d m}\right)_{0}=\lim _{\Gamma \rightarrow 0} \frac{\Gamma}{e^{\Gamma}-1}=\lim _{\Gamma \rightarrow 0} \frac{(\Gamma)^{\prime}}{\left(e^{\Gamma}-1\right)^{\prime}}=1
\end{gathered}
$$

The relationship between the heat transfer coefficients and the derivative of the temperature ratio can then be constructed for the non-transpiration case, where the non-transpiration parameters are indicated by the "**" superscript and the parameters associated with the wall condition are indicated by the " 0 " subscript:

$$
\left(\frac{d \beta}{d m}\right)_{0}^{*}=\Delta^{*}\left(\frac{d \beta}{d y}\right)_{0}^{*}=\Delta^{*} \frac{h_{e f f}^{*}}{k}=1
$$

Using the relationship constructed from above, the mass transfer parameter along the wall location may be re-defined as:

$$
\Gamma=\left(\frac{\rho_{s} w C_{P}}{h_{e f f}^{*}}\right) \frac{\Delta}{\Delta^{*}}
$$

At this point, effective heat transfer coefficient in the transpiration case and the non-transpiration case at the wall location can be compared using above relationships.

$$
\frac{h_{e f f}}{h_{e f f}^{*}}=\left(\frac{h_{e f f}}{k}\right)\left(\frac{k}{h_{e f f}^{*}}\right)=\left(\frac{\partial \beta}{\partial y}\right)_{0} /\left(\frac{\partial \beta}{\partial m}\right)_{0}^{*}=\frac{\left(\frac{\Gamma}{e^{\Gamma}-1}\right)\left(\frac{1}{\Delta}\right)}{1 / \Delta^{*}}=\frac{\left(\frac{\rho_{s} w C_{P}}{h_{e f f}^{*}}\right)}{\exp \left(\frac{\rho_{s} w C_{P}}{h_{e f f}^{*}} \frac{\Delta}{\Delta^{*}}\right)-1}
$$

The transpiration study further suggests that the thickness ratio between the transpiration and the non-transpiration case can be considered as one. ${ }^{13}$ Additionally, the mass transfer in the vertical 
direction is assumed to be governed by mass conservation, such that the regression rate $r_{b}$ and fuel density $\rho_{s}$ can be incorporated into the correction equation for the calculation of the heat transfer coefficient, as follows ${ }^{12}$ :

$$
h_{e f f}=\frac{\rho_{s} r_{b} C_{p}}{\exp \left(\frac{\rho_{s} r_{b} C_{p}}{h_{e f f}^{*}}\right)-1}
$$

Combining the result obtained from film theory and convective heat feedback theory, the final formula for regression rate based on Eq. 3.5 can be constructed:

$$
r_{b}=\frac{\left(h_{x}^{*}+h_{\theta}^{*}\right)}{\rho_{s} C_{p}} \ln \left[1+\frac{C_{p}}{C_{s}} \frac{\left(T_{f}-T_{s}\right)}{\left(T_{s}-T_{i}-\Delta H_{s} / C_{s}\right)}\right]
$$

Further calculations for the non-transpiration heat transfer coefficients $h_{x}^{*}$ and $h_{\theta}^{*}$ for both the axial and tangential flow components are presented in the next section.

\subsection{Calculation of Heat Transfer Coefficients and Friction Factors}

Heat transfer coefficients in the non-transpiration state can be determined using Reynolds' analogy, a theoretical correlation which can be applied towards the flow analysis of a duct (or in this case, port) having a laminar or, in the HRE case, turbulent boundary layer. The well-known correlation relates the heat flux and the shear momentum flux for a particular mass flow of fluid moving above and within the boundary layer. The analogy can be expressed in terms of coefficient of friction for untranspired flow $C_{f}^{*}$, Stanton number $S t$, and Prandtl number Pr :

$$
\frac{C_{f}^{*}}{2}=S t(\operatorname{Pr})^{2 / 3}
$$

Substituting with the parameters that make up St and Pr, one can obtain: 


$$
\frac{C_{f}^{*}}{2}=\left(\frac{h^{*}}{C_{p} \rho_{\infty} u_{\infty}}\right)\left(\frac{C_{p} \mu}{k}\right)^{2 / 3}
$$

By replacing $C_{f}^{*}$ with the untranspired Darcy-Weisbach friction factor $f^{*}$, and further arranging the above equation in terms of the heat transfer coefficient, one can obtain:

$$
h^{*}=\frac{k^{2 / 3} C_{p}^{1 / 3} G}{\mu^{2 / 3}} \frac{f^{*}}{8}
$$

where

$$
f^{*}=4 \times C_{f}^{*}
$$

The heat transfer coefficient can now be expressed in terms of the non-transpiration case for both the axial and tangential flows as shown below:

$$
h_{x}^{*}=\frac{k^{2 / 3} C_{p}^{1 / 3} G_{x}}{\mu^{2 / 3}} \frac{f_{x}^{*}}{8} \quad, \quad h_{\theta}^{*}=\frac{k^{2 / 3} C_{p}^{1 / 3} G_{\theta}}{\mu^{2 / 3}} \frac{f_{\theta}^{*}}{8}
$$

Based on a fully developed turbulent boundary layer, if within the applicable Reynolds number range, Colebrook's equation can be applied in order to estimate the friction factors:

$$
\left(f_{x}^{*}\right)^{-1 / 2}=-2 \log _{10}\left[\frac{2.51}{\operatorname{Re}_{d, x}\left(f_{x}^{*}\right)^{1 / 2}}+\frac{\varepsilon / d_{x}}{3.7}\right],\left(f_{\theta}^{*}\right)^{-1 / 2}=-2 \log _{10}\left[\frac{2.51}{\operatorname{Re}_{d, \theta}\left(f_{\theta}^{*}\right)^{1 / 2}}+\frac{\varepsilon / d_{\theta}}{3.7}\right]
$$

The hydraulic diameter for the axial direction $d_{x}$ is equal to the diameter of the cylindrical fuel grain port, while the effective hydraulic diameter for the tangential direction $d_{\theta}$ is defined based on the effective cross-sectional area of the tangential flow $A_{\theta}$ with respect to the perimeter around the effective cross-sectional area $P_{\theta} \cdot{ }^{11}$ This area is defined based on a finite plane perpendicular to the direction of the effective tangential velocity. It can be expressed in terms of the radial gap between the injector exit and the diameter of the fuel grain, or the assumed 
entrance boundary layer thickness $\delta$, together with the axial length of the fuel grain port $L$, as illustrated in Fig. 4. The definition of $d_{x}$ and $d_{\theta}$ can be expressed as follows ${ }^{11}$ :

$$
d_{x}=d \quad, \quad d_{\theta}=\frac{4 A_{\theta}}{P_{\theta}}=\frac{4(2 \delta \times L)}{(4 \delta+2 L)}
$$

In most cases, the length of the fuel grain is much larger than the gap between the injector and the port diameter. This condition allows the effective hydraulic tangential diameter to be simplified and expressed in terms of the magnitude of the boundary thickness (separation distance) $\delta$ alone. The final simplification of hydraulic tangential diameter is shown below:

$$
d_{\theta}=\frac{4\left(2 \delta \times L_{\rightarrow \infty}\right)}{\left(4 \delta+2 L_{\rightarrow \infty}\right)} \approx \frac{4\left(2 \delta \times L_{\rightarrow \infty}\right)}{\left(2 L_{\rightarrow \infty}\right)} \approx 4 \delta
$$

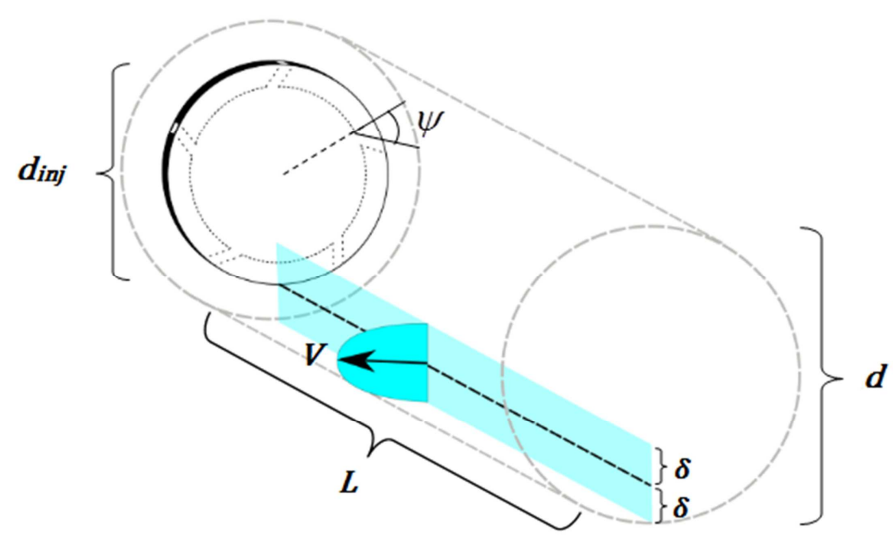

Figure 4: Cross-Section of Port at Head-end and Mid-Body of the Fuel Grain

To further confirm the accuracy of the simplification, the effective hydraulic tangential diameter is plotted with respect to the radial gap at various ratios of $\delta / L$, as shown in Fig. 5 . The simplified calculation remains relatively accurate for the ratio presented in the diagram. In general, a setup of an experimental hybrid rocket engine has a small ratio of $\delta / L$, especially in the case of outward-oriented swirl injectors positioned at the swirl angle $\psi$ as shown in Fig. 4. Therefore, the simplification of the estimation for effective hydraulic tangential diameter is considered reasonable for the type of engines being studied. 


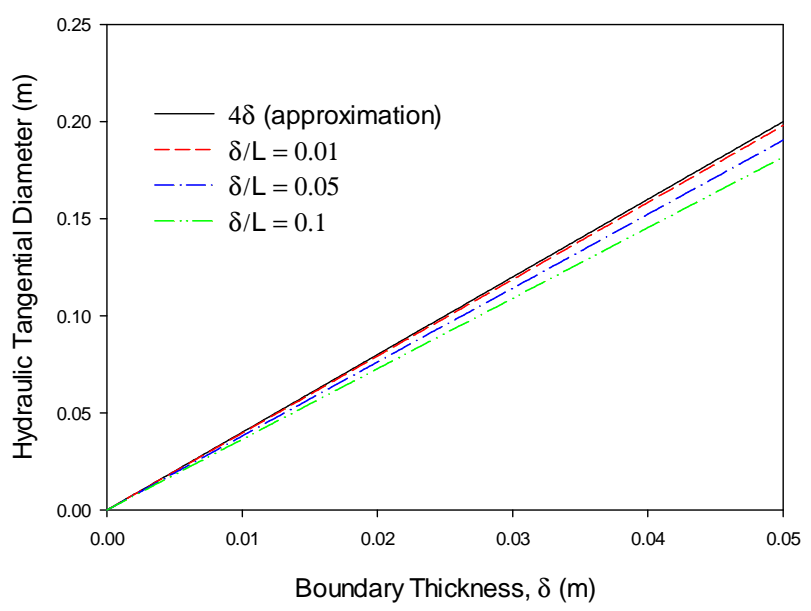

Figure 5: Hydraulic Tangential Diameter Approximation

The next parameter to be considered from Eq. 3.29 is Reynolds number. Reynolds number of axial and tangential flows, $\operatorname{Re}_{d, x}$ and $\operatorname{Re}_{d, \theta}$, can be calculated along with the mass flux terms, $G_{x}$ and $G_{\theta}$, based on the known effective axial and tangential velocity, $U$ and $V$ :

$$
\operatorname{Re}_{d, x}=\frac{G_{x} d_{x}}{\mu}=\frac{\rho U d_{x}}{\mu} \quad, \quad \operatorname{Re}_{d, \theta}=\frac{G_{\theta} d_{\theta}}{\mu}=\frac{\rho V d_{\theta}}{\mu}
$$

Depending on the core flow's Mach number $M a_{p}$, one can further make corrections for the compressibility effect by applying the following correction equation for friction factor.

$$
f_{\text {comp }}=f_{\text {incomp }} /\left[1+\operatorname{Pr}^{1 / 3}\left(\frac{\gamma-1}{2}\right) M a_{p}{ }^{2}\right]
$$

\subsection{Swirling Flow}

In order to improve regression rate, a swirl flow is implemented in a hybrid rocket engine in order to increase the local velocity of the flow with respect to axial mass flux passing over the solid fuel grain. The strength of a swirl flow is commonly expressed using the swirl number $S$, which, from early swirling combustor studies, is the ratio between the axial flux of angular 
momentum and the axial flux of axial momentum multiplying with the radius (radial position from engine chamber centreline, $r_{o}$ ) of the injector. This can be theoretically expressed as ${ }^{14}$ :

$$
S=\frac{\int_{0}^{r_{o}} r^{2} v_{\theta} u_{x} \mathrm{~d} r}{r_{o} \int_{0}^{r_{o}} r\left[u_{x}^{2}+\left(\frac{p-p_{\infty}}{\rho}\right)\right] \mathrm{d} r}
$$

For the purpose of constructing a one-dimensional flow model for the present study, a plug flow is assumed. In other words, the flow being considered has uniform (effective mean) velocity profiles in both axial and tangential directions, where $U$ represents the effective axial velocity and $V$ represents the effective tangential velocity in a constant cylindrical port as shown in Fig. 6. The effective velocity can replace the local velocity in the swirl number expression using the conditions below:

$$
u_{x}=U, \quad v_{\theta}=V
$$

Realizing that there is no pressure variation across a cross-sectional port area (in the radial direction) since both the axial and tangential velocities are set to constant values, one can simplify the expression of swirl number as:

$$
S=\frac{\int_{0}^{r_{o}} r^{2} V U \mathrm{~d} r}{r_{o} \int_{0}^{r_{o}} r U^{2} \mathrm{~d} r}
$$

Integrating both the top and bottom terms in Eq. 3.36, a more simplified relationship between swirl number, effective axial velocity, and effective tangential velocity can be derived:

$$
S=\frac{2}{3} \frac{V}{U}
$$



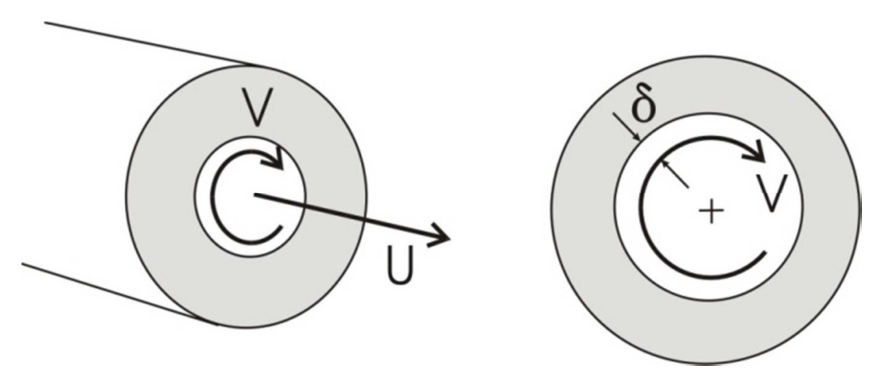

Figure 6: Swirling Flow Inside of a Solid Fuel Grain

Swirl number thus in most cases depends on the ratio between the effective axial and tangential velocity. Moreover, the type of flow governs how swirl number changes with respect to the velocity ratio $V / U$. The approach used to evaluate swirl numbers in this study, for the assumed flow profile, may provide a swirl number that is close to those of other flow variations. For instance, a forced-vortex flow representation, which has a linear tangential velocity profile being maximum at $r_{o}$ and a uniform axial velocity profile, has the swirl number quite close to the model's flow assumption when $V / U$ is less than 1, as shown in Fig. 7. One can expect that other similar flow profiles would also have similar swirl numbers, such that a close estimate of swirl numbers may be obtained using the current flow assumption. Later in Chapter 4, the experimental result shows that the current flow assumption provides a satisfactory prediction for both low and high swirl numbers.

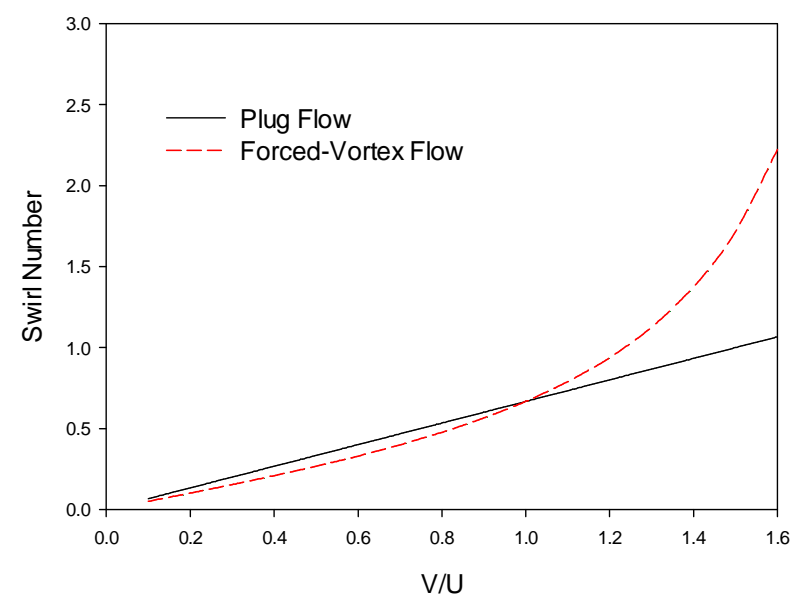

Figure 7: Swirl Number Comparison between Plug Flow and Forced-Vortex Flow 
The swirl number used for the forced-vortex flow discussed above, where the velocity ratio is evaluated based on the maximum tangential velocity, is defined as follows ${ }^{14}$ :

$$
S=\frac{\frac{V_{\max }}{2 U}}{1-\left(\frac{V_{\max }}{2 U}\right)^{2}}
$$

Although one might assume that a swirl flow would behave close to a forced vortex, the flow profile observed from the brief CFD study discussed later in this study suggests that the flow may not necessarily behave exactly as a forced vortex due to various factors. That being said, an averaging approach based on the plug flow assumption is considered to be a reasonable approximation as a reference starting point for evaluating experimental data from various sources where the quoted swirl number is commonly based on an estimate of Eq. 3.34 at the measurement location for the given experimental firing.

An alternative equation for swirl number, for a guided-vane outward-oriented swirl injector, has also been used in the past. The main parameters used for determining the swirl number are the vane (injector port) diameter $d_{h}$, fuel grain port diameter $d$, and the angle of the oxidizer flow injection $\psi$ (a.k.a., swirl angle, as per Fig. 4), which can be expressed as follows ${ }^{15}$ :

$$
S=\frac{2}{3}\left[\frac{1-\left(d_{h} / d\right)^{3}}{1-\left(d_{h} / d\right)^{2}}\right] \tan \psi
$$

With a very small diameter ratio, assuming the ratio $d_{h} / d$ is close to zero in value, the above equation can be simplified further:

$$
S=\frac{2}{3} \tan \psi
$$

The resulting equation is essentially equivalent to Eq. 3.37, and will produce a comparable value for $S$. 
Currently, the modelling and experimental testing of swirl flow in hybrid rocket engines is being studied experimentally by a number of research institutions. However, some of the experimental setups are based on injectors with inward-oriented oxidizer injection, where the flow is radially injected from the peripheral wall via vanes that are tangent to the wall surface. ${ }^{3,16,17}$ The swirl intensity for this type of injection can be represented by the so-called geometrical swirl number $S_{g}$ as shown below:

$$
S_{g}=\frac{\left(R_{x}-R_{\phi}\right) R_{x}}{n R_{\phi}^{2}}
$$

where $R_{x}$ is the overall injector radius, $R_{\phi}$ is the vane radius, and $n$ is the number of vanes. Due to the difference of the injector configurations, this type of swirl flow (and corresponding swirl number formula) is currently presumed not to be suitable for the model prediction technique developed in the present study. Several regression rate estimation models have been proposed by various institutions for this type of swirl injector arrangement. ${ }^{16,17}$

So far, the discussion has been focused on the magnitude of tangential velocity and axial velocity with respect to a swirl number. Realistically, axial, tangential, and radial velocity profiles may vary depending on the strength of the swirl flow as well as the fuel grain geometry. For example when an axial flow is injected, the flow expands to fill the entire cross-section of the port, causing an initial increase of radial velocity. However, the radial velocity in this case is assumed to diminish quickly, so that this effect can be ignored. When a swirl flow is injected, the core flow has much more complicated axial, tangential, and radial velocity profiles. One of the most notable effects of a swirl flow is the inverse pressure variation along the flow centerline. In the case of a swirl number between 0 to 0.6 , the pressure variation is considered relatively weak and will have a small influence on the axial velocity profile. However, when a swirl number is higher than about 0.6 , the pressure variation becomes strong enough to potentially drive the flow back upstream in the vicinity of the head-end injection station, causing a negative axial velocity, and producing a so-called recirculation zone in the pre-combustion chamber region near the engine's head end. ${ }^{18}$ 
Although the velocity profiles close to the center of flow can vary significantly, especially in the case of a strong swirl flow, only the effective velocity components located further away from the centerline of the flow is necessary for determining regression rate via the present approach. Further discussions regarding the flow profiles and effective velocities are provided in Section 3.6 .

\subsection{Related Flow Information}

The properties of the combustion gas produced in hybrid rocket engines are presumed in this study to follow the ideal gas relationship. Density of the gas can be related to chamber pressure and flame temperature as follows:

$$
\rho=\frac{p_{c, s t a t i c}}{R T_{f, \text { static }}}
$$

In the above equation, specific gas constant $R$ is obtained from the universal gas constant divided by the average molecular mass of the propellant gas $\mathrm{M}_{\text {gas }}$ :

$$
R=\frac{8314(\mathrm{~J} / \mathrm{kmol} \cdot \mathrm{K})}{\mathrm{M}_{g a s}}
$$

Additionally, the average absolute viscosity of the propellant gas can be calculated from an experimental correlation, where the main parameters are the flame temperature and the average molecular mass as shown below ${ }^{10}$ :

$$
\mu \cong 8.7 \times 10^{-8}\left(\mathrm{M}_{\text {gas }}\right)^{0.5}\left(T_{f}\right)^{0.65}
$$

\subsection{Summary of Model Calculation Procedure}

A one-dimensional model is created in this thesis for predicting local fuel surface regression rates in swirling-flow hybrid rocket engines. A summary of the calculation procedure for using this model is provided in this section (see Fig. 8). One should note that the prediction requires 
the geometry of the engine, and the propellant properties in both solid and gas phases. The expected principal output of the model is regression rate $r_{b}$ with respect to axial mass flux $G$. Axial mass flux can be a range of values (e.g., from 0 to $1000 \mathrm{~kg} / \mathrm{s}-\mathrm{m}^{2}$ ). For a given or estimated fuel grain port diameter value, the effective axial velocity at given fuel grain axial location can be determined. The angle of oxidizer injection at the head end is used to determine swirl number, which in turn is used for calculating the effective tangential velocity. At this point, the axial flow and the tangential flow components may be considered separately. The first parameter to calculate is Reynolds number, which is necessary for the Colebrook equation that provides friction factor. Depending on the local flow Mach number, friction factor may need to be further corrected for the compressibility effect. Next, the non-transpiration heat transfer coefficient is to be calculated. In the case that no other regression rate mechanism is present save for the flowdependent mechanism under discussion, one can calculate the overall regression rate using Eq. 3.23. However, if a base regression rate is present, the overall regression rate must be calculated iteratively, since the correction equation for heat transfer coefficient is a function of the total regression rate. The regression rate being calculated can be either a mean value or a local value depending on the applications and its usage as discussed in Chapter 4.

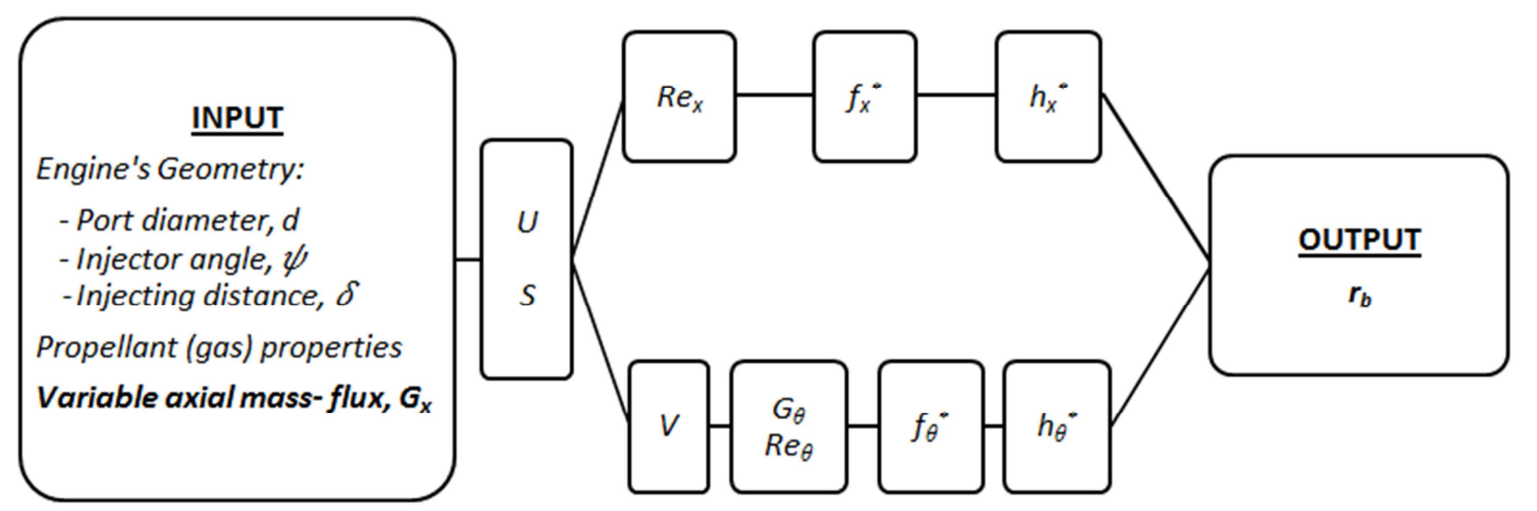

Figure 8: Model Calculation Summary 


\section{Model Applications}

The present model is capable of estimating local fuel surface regression rates where the geometry and gas mass flux at the specified fuel grain location are known. The can be integrated within an overall internal ballistic simulation computer program to obtain the engine's performance over the course of a simulated engine firing, as is done in the RU computer program QSHYB for the quasi-steady internal ballistic performance prediction of various HREs. ${ }^{19,20}$ Shorter approximate analyses can also be done, in order to get quick estimates of performance that may not be overly accurate, but still useful as a guideline.

In order to give the reader an introduction to the elements one would encounter in an internal ballistics calculation procedure, the first example HRE internal ballistics analysis approach presented here is a rough estimation of the rocket engine's performance based on the assumption that the flow velocity inside the engine is relatively low (i.e., having a low flow Mach number) along the length of the fuel grain port, and the chamber pressure is relatively constant, i.e., not dropping too rapidly in moving downstream from the engine head end. In the usual case for chemical rocket engines, the flow is choked at the exhaust nozzle throat, such that chamber pressure can be calculated using the following equation ${ }^{7}$ :

$$
p_{c}=\frac{\dot{m}_{t o t} c^{*}}{A_{t}}
$$

where

$$
c^{*}=\left[\frac{R T_{f}}{\gamma}\left(\frac{\gamma+1}{2}\right)^{\frac{\gamma+1}{\gamma-1}}\right]^{1 / 2}
$$

Characteristic exhaust velocity $c^{*}$ is a function of the properties of the propellant combustion gas. Since the gas properties in the engine chamber are generally considered as being relatively constant, the main parameters allowing for the varying of chamber pressure is the total mass flow rate $\dot{m}_{t o t}$ and nozzle throat area $A_{t}$. The total mass flow is contributed to by the mass flow rate of 
the oxidizer, which is under a controlled injection rate, and mass flow rate of fuel, which is controlled by the burning mechanism (or, if beyond the nominal stoichiometric length, controlled by non-combustive ablation). Normally, the burning mechanism in an HRE is a function of mass flux, which changes as the diameter of the fuel grain port regresses in time. Being able to properly account for the regression rate behavior is a very significant step in determining the engine's performance.

The total mass flow rate can be approximated for a simple cylindrical fuel grain, assuming that the inner diameter of the fuel grain remains relatively constant, and combustion is effective throughout the length of the fuel grain (i.e., $\left.L<L_{s t}\right)^{7}$ :

$$
\dot{m}_{t o t}=\dot{m}_{o}+\dot{m}_{f} \approx \dot{m}_{o}+\rho_{s}\left(\pi d_{x} L\right) \bar{r}_{b}
$$

Thus,

$$
p_{c} \approx\left[\frac{R T_{F}}{\gamma}\left(\frac{\gamma+1}{2}\right)^{\frac{\gamma+1}{\gamma-1}}\right]^{1 / 2} \frac{\dot{m}_{o}+\rho_{s}\left(\pi d_{x} L\right) \bar{r}_{b}}{A_{t}}
$$

The average regression rate in the above equation can be replaced by the regression rate expression outlined in Eq. 3.5 when no base regression rate $r_{o}$ is present, or Eq. 3.6, for the most general case where $r_{o}$ is incorporated into the model, as shown here:

$$
p_{c} \approx\left[\frac{R T_{F}}{\gamma}\left(\frac{\gamma+1}{2}\right)^{\frac{\gamma+1}{\gamma-1}}\right]^{1 / 2}\left[\frac{\dot{m}_{o}}{A_{t}}+\frac{\rho_{s}\left(\pi d_{x} L\right)}{A_{t}}\left(r_{o}+\frac{\left(h_{x}+h_{\theta}\right)\left(T_{f}-T_{s}\right)}{\rho_{s}\left[C_{s}\left(T_{s}-T_{i}\right)-\Delta H_{s}\right]}\right)\right]
$$

Next, thrust can be ascertained by the following equation ${ }^{7}$ :

$$
F=C_{F} A_{t} p_{c}
$$


which is the rocket thrust equation in terms of the thrust coefficient $C_{F}$. Via substitutions, one can show that

$$
F=\left[\frac{2 \gamma^{2}}{\gamma-1}\left(\frac{2}{\gamma+1}\right)^{\frac{\gamma+1}{\gamma-1}}\left(1-\left(\frac{p_{e}}{p_{c}}\right)^{\frac{\gamma-1}{\gamma}}\right)\right]^{\frac{1}{2}} A_{t} p_{c}+\left(p_{e}-p_{\infty}\right) A_{e}
$$

where

$$
p_{e}=p_{c}\left[1+\frac{\gamma-1}{2} M a_{e}^{2}\right]^{\frac{-\gamma}{\gamma-1}}
$$

and the nozzle exit's flow Mach number can be determined from:

$$
\frac{A_{t}}{A_{e}}=\frac{M a_{e}}{M a_{t}}\left[\frac{2+(\gamma-1) M a_{t}^{2}}{2+(\gamma-1) M a_{e}^{2}}\right]^{\frac{\gamma+1}{2(\gamma-1)}}
$$

where flow Mach number at the throat location is equal to 1 due to the choked condition, and the area ratio between the nozzle throat and the nozzle exit is known. The above analysis is useful to get a quick estimation for the performance of a hybrid rocket engine. It also demonstrates an overall calculation procedure, as well as how the regression rate estimation model is being applied to an internal ballistic analysis.

A numerical analysis is another approach used to evaluate engine performance, where the results obtained are potentially more accurate. One-dimensional quasi-steady-state equations described by Greatrix ${ }^{7}$ allow for time-independent solutions for a flow inside of a hybrid rocket engine. Two sets of the mass, momentum and energy conservation equations are presented for the core flow and the fuel particles which are being transferred into and then traveling down the core flow. The solutions for the core flow (where the gas component is produced by the combustion of the solid fuel reacting with the oxidizer above the fuel surface) moving from the head-end location to the nozzle are presented below. ${ }^{7}$ 


$$
\begin{gathered}
\frac{d(\rho u)}{d x}=-\frac{1}{A} \frac{d A}{d x} \rho u+\left(1-\alpha_{p}\right) \rho_{s} \frac{4 r_{b}}{d_{x}}-\left(\frac{4 r_{b}}{d_{x}}\right) \rho \\
\frac{d\left(\rho u^{2}+p\right)}{d x}=-\frac{1}{A} \frac{d A}{d x} \rho u^{2}-\left(\frac{4 r_{b}}{d_{x}}\right) \rho u-\rho a_{x}-\frac{\rho_{p}}{m_{p}} D \\
\frac{d(\rho u E+u p)}{d x}=-\frac{1}{A} \frac{d A}{d x}(\rho u E+u p)-\left(\frac{4 r_{b}}{d_{x}}\right) \rho E \\
+\left(1-\alpha_{p}\right) \rho_{s} \frac{4 r_{b}}{d}\left(C_{p} T_{F}+\frac{w^{2}}{2}\right)-\rho u a_{x}-\frac{\rho_{p}}{m_{p}}\left(u_{p} D+\dot{Q}\right)
\end{gathered}
$$

The solutions for mass, momentum and energy of fuel particles entering into the core flow are as shown below. ${ }^{7}$

$$
\begin{gathered}
\frac{d\left(\rho_{p} u_{p}\right)}{d x}=-\frac{1}{A} \frac{d A}{d x} \rho_{p} u_{p}+\alpha_{p} \rho_{s} \frac{4 r_{b}}{d_{x}}-\left(\frac{4 r_{b}}{d_{x}}\right) \rho_{p} \\
\frac{d\left(\rho_{p} u_{p}^{2}\right)}{d x}=-\frac{1}{A} \frac{d A}{d x} \rho_{p} u_{p}^{2}-\left(\frac{4 r_{b}}{d_{x}}\right) \rho_{p} u_{p}-\rho_{p} a_{x}-\frac{\rho_{p}}{m_{p}} D \\
\frac{d\left(\rho_{p} u_{p} E_{p}\right)}{d x}=-\frac{1}{A} \frac{d A}{d x}\left(\rho_{p} u_{p} E_{p}\right)-\left(\frac{4 r_{b}}{d_{x}}\right) \rho_{p} E_{p} \\
+\alpha_{p} \rho_{s} \frac{4 r_{b}}{d}\left(C_{m} T_{F}+\frac{w^{2}}{2}\right)-\rho_{p} u_{p} a_{x}+\frac{\rho_{p}}{m_{p}}\left(u_{p} D+\dot{Q}\right)
\end{gathered}
$$

The model for regression rate estimation can be applied for the $r_{b}$ terms in Eq. 4.10 to 4.15 in the same manner as done earlier for Eq. 4.5. When an additional base regression rate component $r_{o}$ is to be accounted for the regression rate cannot be determined directly (explicitly); therefore, as an additional step, an iterative calculation procedure must be implemented for estimating overall regression rate $r_{b}$ in those cases. 
Further considerations are required for an even more accurate analysis. One important issue that may be encountered in a hybrid rocket engine is when the stoichiometric length is substantially shorter than the actual length of the fuel grain $\left(L_{s t}<L\right)$. Since the aft part of the fuel grain does not react in the combustion process in this scenario, the regression rate mechanism over the aft part of the grain is assumed to be mainly driven by non-combustive ablation. The length of reactive fuel grain can be calculated by first obtaining the ideal mass flow rate of fuel based on the effective stoichiometric mixture ratio $r_{s t}$ of the propellant for the known mass flow of the oxidizer. The stoichiometric length can then be approximated from the internal port diameter and cross-sectional port area of the fuel grain, and the nominal regression rate along the axial length, as shown below:

$$
L_{s t} \approx \frac{G_{o} A_{p}}{r_{s t} \rho_{s} r_{b} \pi d_{x}}
$$

The regression of the aft grain section that undergoes non-combustive ablation can be calculated using ablation rate $e_{s}$. It is estimated as was done for regression rates presented earlier, i.e., using convective heat feedback theory, with the resulting equation as shown below ${ }^{7}$ :

$$
e_{s}=\frac{h_{e f f}^{*}}{\rho_{s} C_{p}} \ln \left[1+\frac{C_{p}}{C_{s}} \frac{\left(T_{\infty}-T_{d s}\right)}{\left(T_{d s}-T_{i}-\Delta H_{s} / C_{s}\right)}\right]
$$

Once the regression rate profile along the axial length of the fuel grain is obtained, thrust can be calculated in the same manner as previously discussed. Numerical analysis based on the quasisteady-state internal ballistic equations described above has been conducted for various engines as part of this and other RU studies. Some relevant sample numerical results ${ }^{19}$ are presented in Appendix A, for the benefit of the reader. 


\section{Experimental Verification}

The regression rate model developed for this study can be verified by comparing the accuracy of the predicted results to actual experimental data for fuel regression rates. Test data, obtained from the firing of lab-scale experimental hybrid rocket engines (using head-end swirl injectors) at various research institutions around the world, are presented in various technical papers available in the literature. ${ }^{4,5}$ Eight engine configurations are considered for comparison here, where the engines are named from $\mathrm{A}$ to $\mathrm{H}$ as listed in Table 1, along with the respective engine and gas properties. The propellant combinations used in the experimental cases include:

1. Paraffin/GOx - paraffin wax (type FT-0070) as fuel; gaseous oxygen as oxidizer

2. $\mathrm{HDPE} / \mathrm{N}_{2} \mathrm{O}$ - high density polyethylene as fuel; nitrous oxide as oxidizer

3. HTPB/GOx - hydroxyl terminated polybutadiene as fuel; gaseous oxygen as oxidizer

The focus for each configuration is the various swirl numbers being used for the respective test firings, where $S$ is dictated by the angles of injection of the oxidizer injectors. The values for $S$ range from 0 for an axial flow with a tangential injection angle $\psi$ of $0^{\circ}$, to a value of 1.15 for a strong swirl flow with $\psi$ of $60^{\circ}$.

Some differences between the predictive model's reference framework, and the actual experiments and how they are conducted, should be noted. The predictive model is based on a set of inputs where the engine geometry as well as the gas properties, which are assumed to be constant, or relatively constant, at the specific location and time of interest. However, hybrid rocket engines do have their performance change as a result of the regressing fuel profile, the changing $\mathrm{O} / \mathrm{F}$ ratio (a.k.a., mixture ratio $r$ which in turn affects stoichiometric length $L_{s t}$ ), and the corresponding flow field. Therefore, a closer match between the predictive model and the experimental cases can commonly be better obtained when the engine firing time is short, or when the data is being collected and evaluated with respect to a relatively short period of time. In the case of some experimental measurement techniques, the same by analogy applies to the spatial length of the location of interest, i.e., the shorter the length, the better for comparing better to the predictive model. 
Based on the above discussion, some discrepancies are to be expected, at least in part due to the difference between the ideal behavior that is the underlying premise the predictive model, versus the practical factors of the way the experimental data is being measured and evaluated. Other factors, e.g., those related to the given experimental apparatus itself, can also lead to wider differences between theory and experiment.

Table 1: Engine Characteristics of Experimental Hybrid Rocket Engines

\begin{tabular}{lllllllll}
\hline Engine: & $\mathrm{A}$ & $\mathrm{B}$ & $\mathrm{C}$ & $\mathrm{D}$ & $\mathrm{E}$ & $\mathrm{F}$ & $\mathrm{G}$ & $\mathrm{H}$ \\
\hline$\psi(\mathrm{deg})$ & 0 & 30 & 45 & 0 & 30 & 60 & 0 & 55 \\
$S$ & 0 & 0.385 & 0.667 & 0 & 0.385 & 1.156 & 0 & 0.952 \\
$d \quad(\mathrm{~cm})$ & 2 & 2 & 2 & 5.1 & 5.1 & 5.1 & 2.2 & 2.2 \\
$d_{\theta}(\mathrm{cm})$ & 1 & 1 & 1 & 3.6 & 3.6 & 3.6 & 2 & 2 \\
$\rho_{s}\left(\mathrm{~kg} / \mathrm{m}^{3}\right)$ & 920 & 920 & 920 & 920 & 920 & 920 & 920 & 920 \\
$C_{s}(\mathrm{~J} / \mathrm{kg}-\mathrm{K})$ & 2100 & 2100 & 2100 & 920 & 920 & 2100 & 2100 & 2100 \\
$T_{f}(\mathrm{~K})$ & 3500 & 3500 & 3500 & 2600 & 2600 & 2600 & 3650 & 3650 \\
$T_{s}(\mathrm{~K})$ & 400 & 400 & 400 & 950 & 950 & 950 & 950 & 950 \\
$T_{i}(\mathrm{~K})$ & 293 & 293 & 293 & 293 & 293 & 293 & 293 & 293 \\
$C_{p}(\mathrm{~J} / \mathrm{kg}-\mathrm{K})$ & 2000 & 2000 & 2000 & 1920 & 1920 & 1920 & 1920 & 1920 \\
$R(\mathrm{~J} / \mathrm{kg}-\mathrm{K})$ & 385 & 385 & 385 & 320 & 320 & 320 & 320 & 320 \\
$\mu\left(\mathrm{N} \mathrm{s} / \mathrm{m}^{2}\right)$ & $2.5 \times 10^{-5}$ & $2.5 \times 10^{-5}$ & $2.5 \times 10^{-5}$ & $8.0 \times 10^{-5}$ & $8.0 \times 10^{-5}$ & $8.0 \times 10^{-5}$ & $8.0 \times 10^{-5}$ & $8.0 \times 10^{-5}$ \\
$k(\mathrm{~W} / \mathrm{m}-\mathrm{K})$ & 0.2 & 0.2 & 0.2 & 0.2 & 0.2 & 0.2 & 0.2 & 0.2 \\
$\varepsilon(\mu \mathrm{m})$ & 10 & 10 & 10 & 5 & 5 & 5 & 5 & 5 \\
\hline
\end{tabular}




\section{$\underline{\text { Engine A-C }}$}

The data obtained from the first three engines, A, B and C, are based on the same experimental apparatus using paraffin wax and GOx (gaseous oxygen) as the propellant. ${ }^{4}$ The starting fuel port diameter for these experiments is $2 \mathrm{~cm}$. From the injector and fuel port dimensions the effective tangential hydraulic diameter is calculated to be around $1 \mathrm{~cm}$. Three tangential injectors were used including $0^{\circ}, 30^{\circ}$, and $45^{\circ}$ injection angles for engine $\mathrm{A}, \mathrm{B}$, and $\mathrm{C}$ respectively. These correspond to swirl numbers of $0,0.385$, and 0.667 . As previously described, the injector used for engine A leads to an axial flow, while engine B generates a weak swirl flow, and engine C generates a stronger swirl flow. Fig. 9 shows the comparison between the regression rates predicted by the model versus the regression rate data obtained from the test firing of engine A, $\mathrm{B}$, and C. As illustrated in the graph, the predicted values compare quite well to the experimental results. One can observe a slightly over-predicted result for case A, and a slightly underpredicted result for case $\mathrm{C}$. The baseline run used in this case is the non-swirl configuration of engine A.

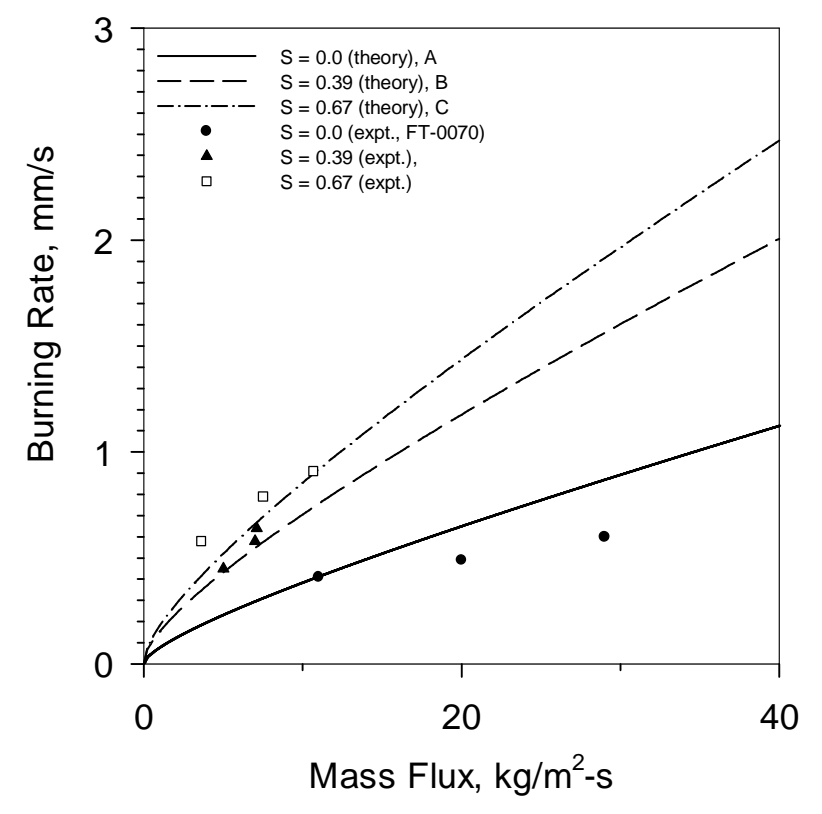

Figure 9: Predictive Model Results and Corresponding Experimental Data, ${ }^{4}$ for Fuel Regression Rate as Function of Mass Flux and Swirl Number, for Same Paraffin/GOx Propellant 


\section{Engine D-F}

The recorded data of regression rates for engine $\mathrm{D}, \mathrm{E}$, and $\mathrm{F}$ are obtained based on a set of differing experimental setups for one hybrid rocket engine. ${ }^{5}$ The propellant combination used for these engine setups is HDPE and $\mathrm{N}_{2} \mathrm{O}$. The initial port diameter or the fuel grain inner diameter is $5.1 \mathrm{~cm}$, while the effective hydraulic tangential diameter is calculated to be $3.6 \mathrm{~cm}$. Similarly to the previous case, different swirl injector settings were used between the three engine configurations. These include $0^{\circ}, 30^{\circ}$, and $60^{\circ}$, where the corresponding swirl numbers are 0 , 0.385 , and 1.156, respectively. For case D, the flow is considered to be an axial (non-swirl) flow. Based on the stated swirl numbers, the flow characteristics in engine $\mathrm{E}$ and $\mathrm{F}$ are a weak swirl flow and a strong swirl respectively.

Only a single experimental data point is available for each of the experimental engine setups (the origin, zero, might qualify as a second data point); therefore, the similarity quality of the regression rate trend lines is not able to be discussed at length. Nevertheless, the data shown for both axial and tangential cases seem to be very close to the predictive model curves. This further lends support to the validity of the current calculation model.

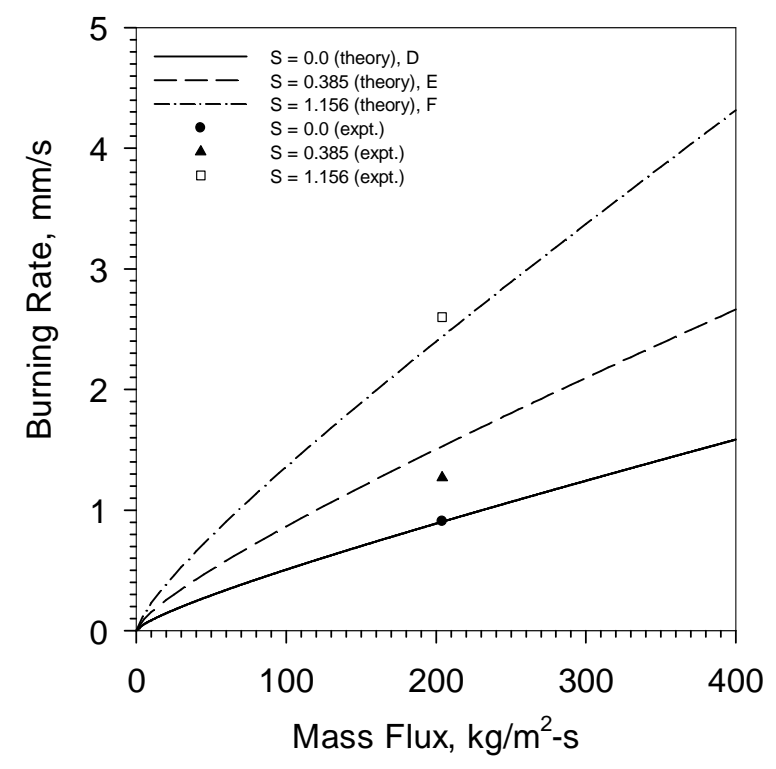

Figure 10: Predictive Model Results and Corresponding Experimental Data, ${ }^{5}$ for Fuel Regression Rate as Function of Mass Flux and Swirl Number, for Same HDPE/ $\mathrm{N}_{2} \mathrm{O}$ Propellant 


\section{Engine $\mathrm{G}$ and $\mathrm{H}$}

The recorded data for regression rates for engine configurations $\mathrm{G}$ and $\mathrm{H}$ are also obtained from the differing experimental setups of the same hybrid rocket engine. ${ }^{6}$ The propellant combination used for both of the engine setups is HTPB and GOx. Initially, the port diameter is $2.2 \mathrm{~cm}$, while the effective tangential hydraulic diameter is around $2 \mathrm{~cm}$. Each of the engines was tested using different injectors, where engine setup $\mathrm{G}$ uses an axial flow injector at $0^{\circ}$ and engine $\mathrm{H}$ uses a swirl injector at $55^{\circ}$. These injector angles correspond to swirl numbers of 0 and 0.952 , respectively. The injector in case G provides an axial (non-swirl) flow, while the injector in case $\mathrm{H}$ provides a strong swirl flow.

The comparisons of the experimental data with the predicted results show more noticeable discrepancies relative to the previous engines. However, the predicted results still follow the general trend of the experimental data.

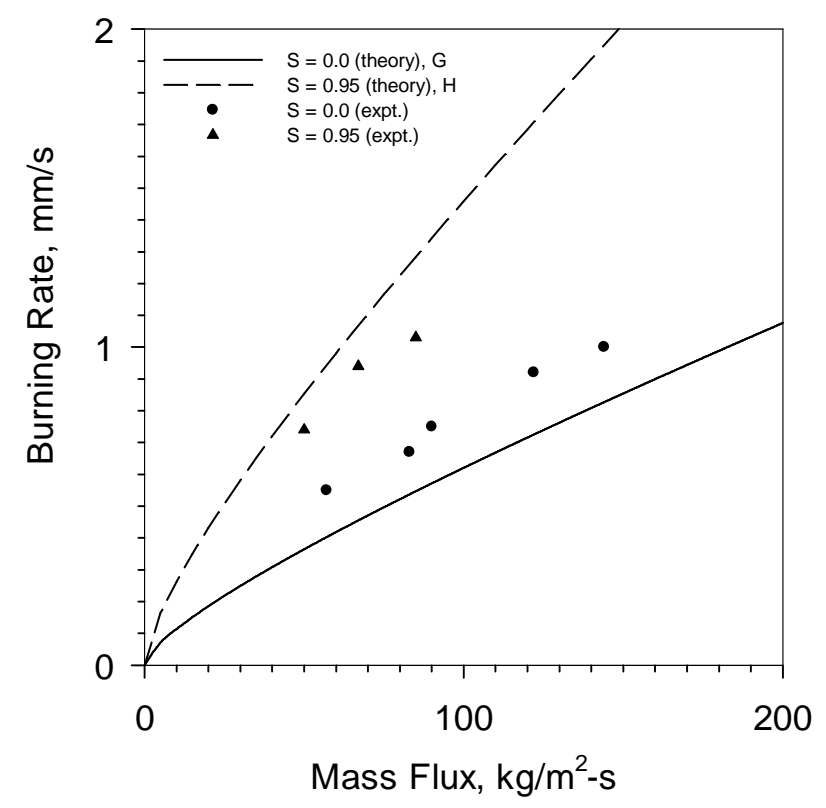

Figure 11: Predictive Model Results and Corresponding Experimental Data, ${ }^{6}$ for Fuel Regression Rate as Function of Mass Flux and Swirl Number, for Same HTPB/GOx Propellant 


\section{$\underline{\text { Further Discussion }}$}

In general, the results of the various comparisons are, for the most part, quite satisfactory. The predictive model follows the trend of the experimental data with some varying degrees of accuracy. The magnitude of some discrepancies may originate in part due to some measurement techniques and apparatus issues discussed earlier at the beginning of the chapter. Differences between the incoming oxidizer mass flux $G_{O}$ and the actual axial mass flux $G_{x}$ at the point of measurement may also be an issue contributing to some apparent discrepancies, if the regression rate experimental data is framed, as is commonly done, in terms of $G_{O}$.

Additionally, the discrepancies observed in each of the engine configurations are expected to be due to combined factors, where the sources of the discrepancies may be difficult to clarify unless each of the engine cases are being examined closely along with additional research effort, which could be a part of future work in this area. To give an example, an engine undergoing a long firing time would result in a certain profile of regression rates depending on the continuouslychanging mixture ratios and mass flux (due to both the effects of fuel mass addition and changes of the fuel grain geometry). This could either be a positive or a negative effect to the regression rate measured in an experiment versus the prediction. Furthermore, an engine with a long fuel grain might also experience the region with non-combustive ablation beyond the stoichiometric length which would lower the average result of regression rate. Rather than try to identify each of the effects in order to describe quantitatively the discrepancies between the experimental results (generated at other research institutions) and the corresponding prediction, this research mainly focuses on the general observed trends arising from the framework of presumed mean effective flows (as described in Chapter 3). 


\section{Computational Fluid Dynamic Analysis of a Simplified Engine Flow Model}

A complementary CFD mini-study on swirling flows in HREs was undertaken as part of this thesis effort. A number of numerical simulation runs were to be conducted to potentially help better understand the flow patterns and behavior inside the fuel grain port of a simplified engine flow model, in the presence (and absence) of swirl and transpiration. The implementation of this type of numerical analysis requires the capabilities of a CFD software package that can model three-dimensional swirl flows, flows which are quite complex in nature. For this purpose, ANSYS Fluent ${ }^{\dagger}$ was selected for this mini-study. Major characteristics of a swirling-flow hybrid rocket engine that were to be investigated included: decay of swirl intensity moving downstream from the engine head end (an issue raised by some HRE researchers, with respect to estimating local $S$ values), and the effect of the various flow features, including transpiration, on the axial and tangential velocity components in a more comprehensive model representation, as one moves downstream.

The three-dimensional simplified physical model of a hybrid rocket engine was created using a cylindrical duct with an exhaust nozzle at one end, where the contracting and expanding sections of the nozzle were made of cone walls. Some of the physical effects that one associates with HREs, such as the time-dependent regression of the fuel grain surface, cannot (readily) be incorporated into the simulation, and hence were not included in the present effort. Another physical effect that could not readily be included is the combustion process at and above the solid fuel surface, given the limitations of the software package available. Mass transpiration of gas from the bounding inner port surface could be modelled in a straightforward non-reactive cold-flow (albeit, rudimentary) fashion. The present flow model has two mass-flow inlets, including the oxidizer inlet at the head-end, and the fuel (transpiration) inlet along the specified peripheral area moving downstream from the head end, where the dimensions follow the diagram as shown in Fig. 12. The inspiration for the chosen flow model geometry (although the geometry has been modified) was a small hybrid rocket engine which had been used in a swirl-flow regression rate study at Arizona State University. ${ }^{21}$ Additionally, a second simplified engine

\footnotetext{
${ }^{\dagger}$ ANSYS Fluent: A commercial simulation environment mandated for comprehensive Computational Fluid Dynamics (CFD) analyses

${ }^{\ddagger}$ Cold-Flow: A non-combustive flow commonly used in experimentation of a rocket engine instead of test firings
} 
model was constructed for testing the baseline behavior of the simulated flow. This is discussed further in Section 6.1.

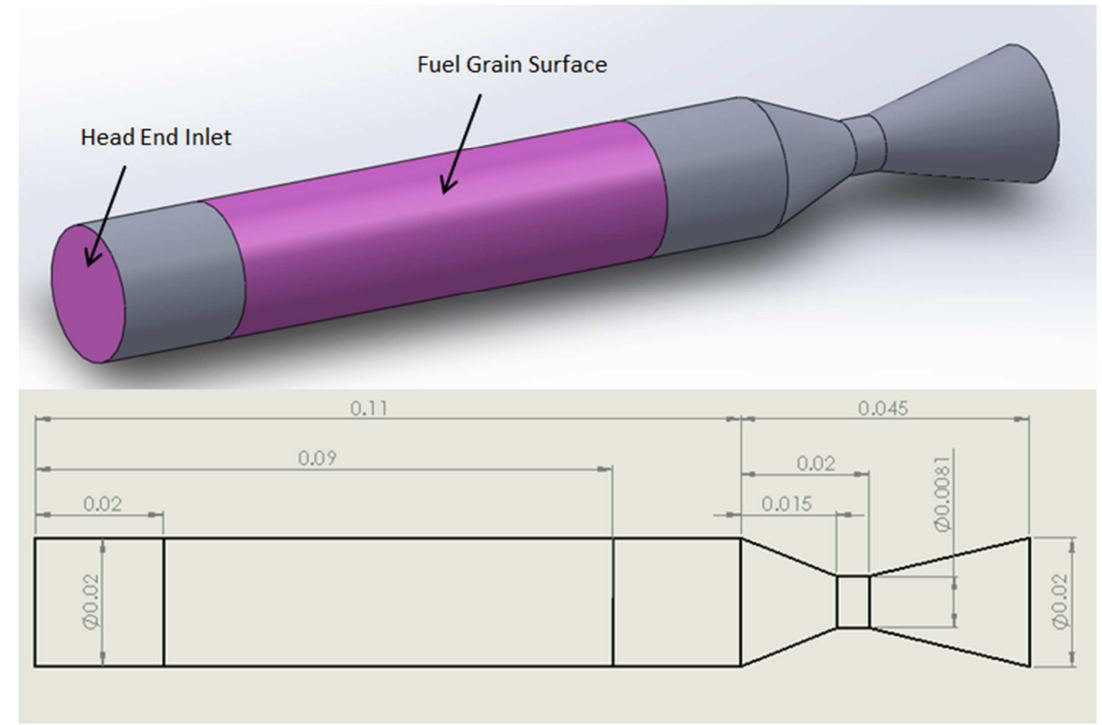

Figure 12: CFD Model for Hybrid Rocket Engine (Dimensions in Meters)

In the full axisymmetric engine model shown in Fig. 12, wall sections before and after the fuel grain are attached, such that the transition zones due to the inlet injection and the nozzle contraction would take place within these walled regions. The monitored fuel port region is located along the axial length between $0.02 \mathrm{~m}$ to $0.09 \mathrm{~m}$, which is also the region where the transpiration mass flow tied to the regression of the fuel grain is being radially implemented. As a simpler cold-flow representation of the problem, the primary flow coming from the head-end injector inlet and the transpiration flow coming from the fuel grain surface are assumed to be a propellant gas with properties as shown in Table 2. Ideally, the head-end inlet should be similar to the injector shown in Fig. 4 in order to have a swirl flow entering with a boundary thickness $\delta$, which would be much closer to the real flow profile. However, this flow injection approach is expected to further complicate the flow profile; hence, the effects that need to be investigated would become less obvious. As a result, the simplified inlet is used, where the constant injected axial and tangential profiles occupy the entire cross-sectional area at the inlet, which is now less realistic for the HRE application. 
Table 2: Propellant Gas and Initial Properties

\begin{tabular}{ll}
\hline Gas Properties & Initial Values \\
\hline$\rho \quad\left(\mathrm{kg} / \mathrm{m}^{3}\right)$ & $\left(\frac{p_{c, \text { static }}}{R T_{f}}\right)$ \\
$T_{f}(\mathrm{~K})$ & 3000 \\
$C_{p}(\mathrm{~J} / \mathrm{kg}-\mathrm{K})$ & 1920 \\
$\gamma$ & 1.2 \\
$k \quad(\mathrm{~W} / \mathrm{m}-\mathrm{K})$ & 0.2 \\
$\mu \quad(\mathrm{kg} / \mathrm{m}-\mathrm{s})$ & $8 \times 10^{-5}$ \\
$M \quad(\mathrm{~kg} / \mathrm{kmol})$ & 25.98 \\
\hline
\end{tabular}

Some other important features related to the model setup in the software are listed below:

- Type of simulation: steady state, viscous, compressible, ideal gas

- Number of nodes: 75,712

- Node condensation: centerline and boundary layers along the walls

- Calculation model: convective heat transfer and $k-\varepsilon$ turbulent

- Mode : density-based computation

- Baseline reference pressure: $101300 \mathrm{~Pa}$

Note that the numbers of nodes being used for the flow are relatively small (essentially, to obtain "reasonable" computational turn-around times), so some level of error would be expected. One can note that the $k-\varepsilon$ turbulence model is used largely due to its demonstrated past reliability for conventional flow modelling. Other methods, such as one based on a Reynolds stress model, could potentially provide a higher accuracy; however, substantial additional computational time is needed as a trade-off, such that they would not be suitable for the current mini-study. Since the goal of this mini-study is simply to observe the general trend of the flow behavior, less emphasis is put on the effort to obtain or validate the accuracy of the results. Additionally, as a notable example, a more elaborate CFD study of a swirling-flow HRE has been conducted at the University of Padua, for readers that are interested in more realistic flow representations. ${ }^{22}$ 


\subsection{Pre-Analysis}

Prior to running the full engine model simulation runs, more simplified cases were evaluated in order to understand the baseline mechanisms of swirl flows, e.g., without any interference from transpiration. A second three-dimensional model was set up based on the full model shown earlier, with some modifications. The fuel section inner surface is set as being a solid (nontranspired) wall in this case, where the axial length of the section before the nozzle is reduced from $0.011 \mathrm{~m}$ to $0.09 \mathrm{~m}$, since no transition zone at the entrance is expected. This also helps towards reducing some of the computational time. The mass flow rate at the head-end injector inlet is set to $0.12716 \mathrm{~kg} / \mathrm{s}$, which is equal to the total mass flow rate used later in the full model. The static chamber pressure is preliminarily set to a value at $3.793 \mathrm{MPa}(550.13 \mathrm{psi})$ at the beginning of the simulation run, this value being the nominal quasi-equilibrium chamber pressure expected under steady state operation. This pressure also reflects on the design of the model geometry, particularly the area ratio at the contracting section of the choked nozzle. The incoming head-end inlet mass flow is:

$$
\dot{m}=\left(\frac{p_{c, \text { static }}}{R T_{F}}\right) U A_{p}=\left(\frac{p_{c, \text { static }}}{R T_{F}}\right) M a_{p} \sqrt{2 R T_{f}} A_{p}
$$

For a known port area $A_{p}$, the area ratio of the contracting nozzle can be evaluated, since Mach number at the throat location $M_{t}$ is equal to 1 :

$$
\frac{A_{t}}{A_{p}}=\frac{M a_{p}}{M a_{t}}\left[\frac{2+(\gamma-1) M a_{t}^{2}}{2+(\gamma-1) M a_{p}^{2}}\right]^{\frac{\gamma+1}{2(\gamma-1)}}
$$

In this pre-analysis study, three cases of turbulent flows are to be evaluated (by altering the inlet condition): an axial (non-swirl) flow, a weak swirl flow, and a strong swirl flow, as discussed in the sections below for Case 1, 2, and 3 respectively. 


\section{Case 1: Axial Flow Without Transpiration}

The first case to be considered is an axial (non-swirl) flow without transpiration. It is used as a reference case to understand the baseline behavior of the flow inside of the fuel grain port, while the flow characteristics are restricted by a choked-flow nozzle. The monitored area for this simulation starts from the inlet at $0 \mathrm{~m}$ to the axial location right before the nozzle at $0.09 \mathrm{~m}$. This includes the transition region of the flow before entering the nozzle (i.e., at around 0.08 to 0.09 $\mathrm{m})$, which is to be neglected for this analysis. The simulation results displayed in Fig. 13 show the streamline profile, the pressure contour profile, and the axial velocity contour profile. Additional detailed results of the pressure and velocity profiles are provided in Figs. 14 to 16.

Although both pressure and velocity are relatively constant throughout the fuel grain port, small changes can be observed. One might presume that the thin layer near the wall, where the velocity profile changes more significantly, is representative of the lower portion of the turbulent boundary layer zone (nearer to the surface) and the laminar sublayer very close to the fuel surface, with the shear flow that results from wall friction. Since the mass (of the gas flow) is moving slower nearer to the wall, the mass further away from the wall, at a relatively constant gas density, must be faster in order to deliver the specified overall mass flow rate downstream. This effect then to some degree causes the pressure to slightly drop as shown in Fig. 16, in addition to the pressure drop one associates with viscous flow losses in channels.

As a quick check, the pressure calculated by an analytical approach based on the nozzle throat geometry shown in Eqs. 6.1 to 6.2, or the aforementioned preliminary design pressure at 3.793 $\mathrm{MPa}$, is compared to the pressure profile given by the software. This comparison approach is taken since pressure is not fixed by the defined surfaces' boundary conditions, whereas other parameters, such as the mass flow rate and associated gas properties, are fixed. The simulation results provide that the pressure within the fuel grain port ranges between $3.759 \mathrm{MPa}$ near the nozzle to $3.761 \mathrm{MPa}$ near the head-end inlet, which are reasonably close to the design value. Therefore, the resulting simulated flow displayed for this case shows some consistency with original calculations. For other cases, Case 2 to 6 , the accuracy is expected to drop due to the 
complexity of the flow; however, it is presumed that the simulation models should still be able to represent the general behavior of the flow.

Also, some possible evidence of computational errors in the flow solution at the inlet location may be observed near the location of the wall, as shown in Fig. 14. However, these errors are assumed to have a small effect on the overall computational results as relates to qualitative trends. Other cases under study here appear to have these small errors present as well.

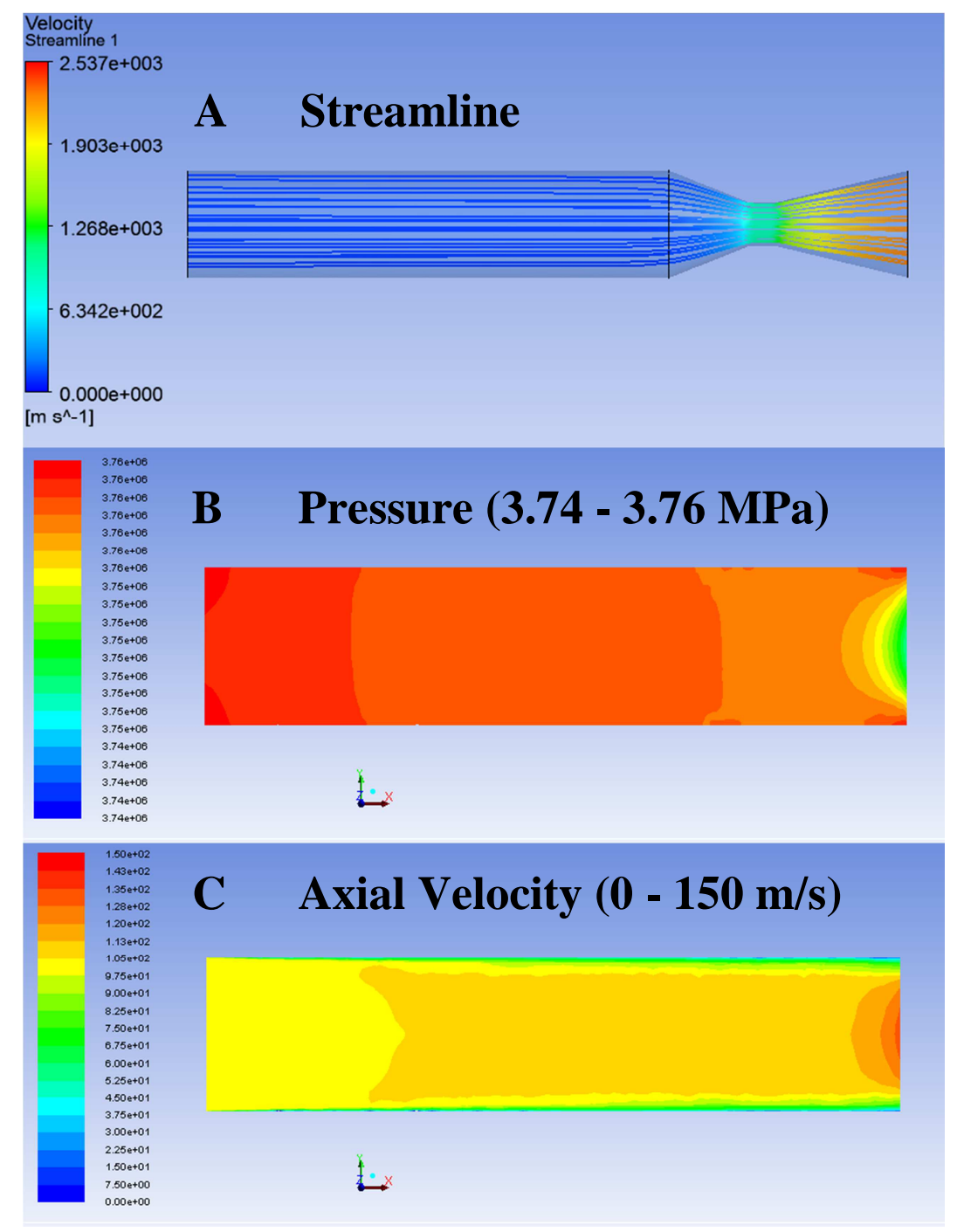

Figure 13: Case 1 - Streamline, Pressure, and Axial Velocity Profiles for Engine 


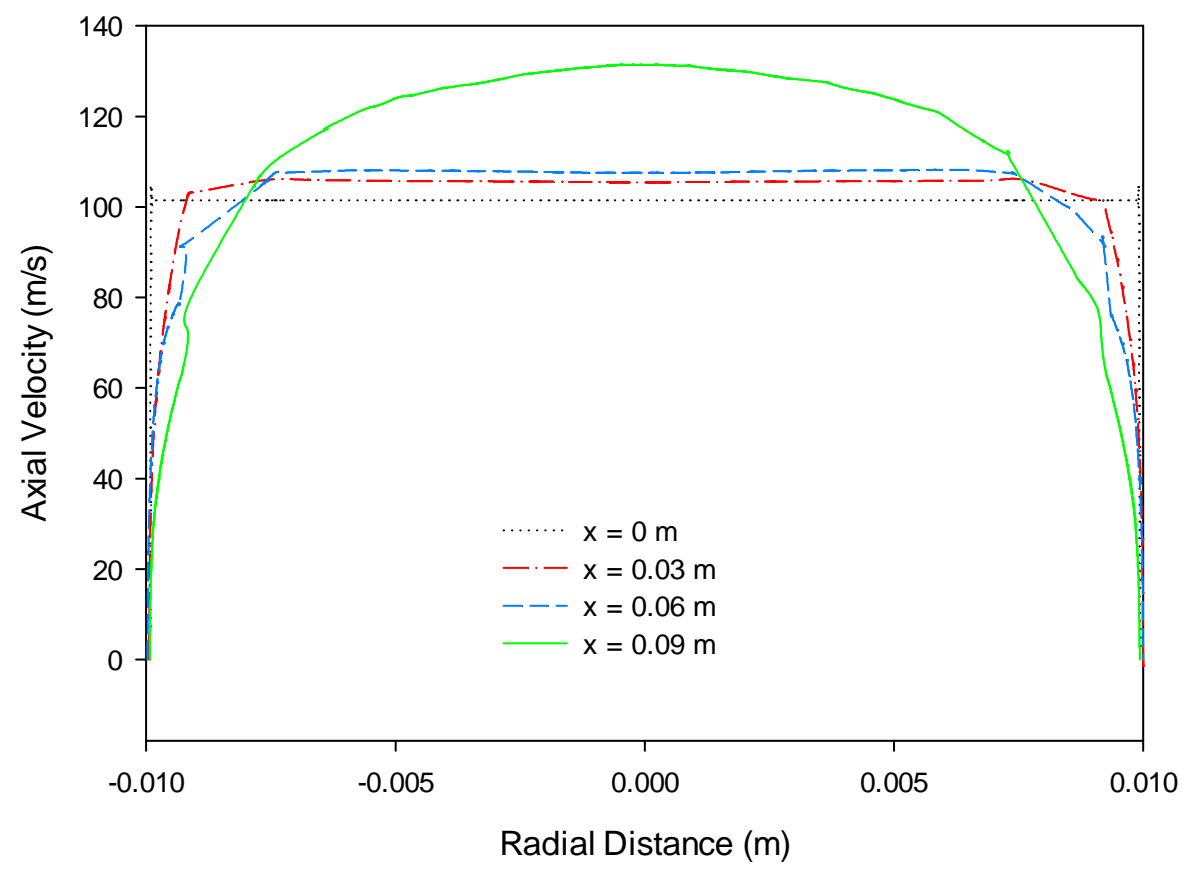

Figure 14: Case 1 - Detailed Axial Velocity Profiles

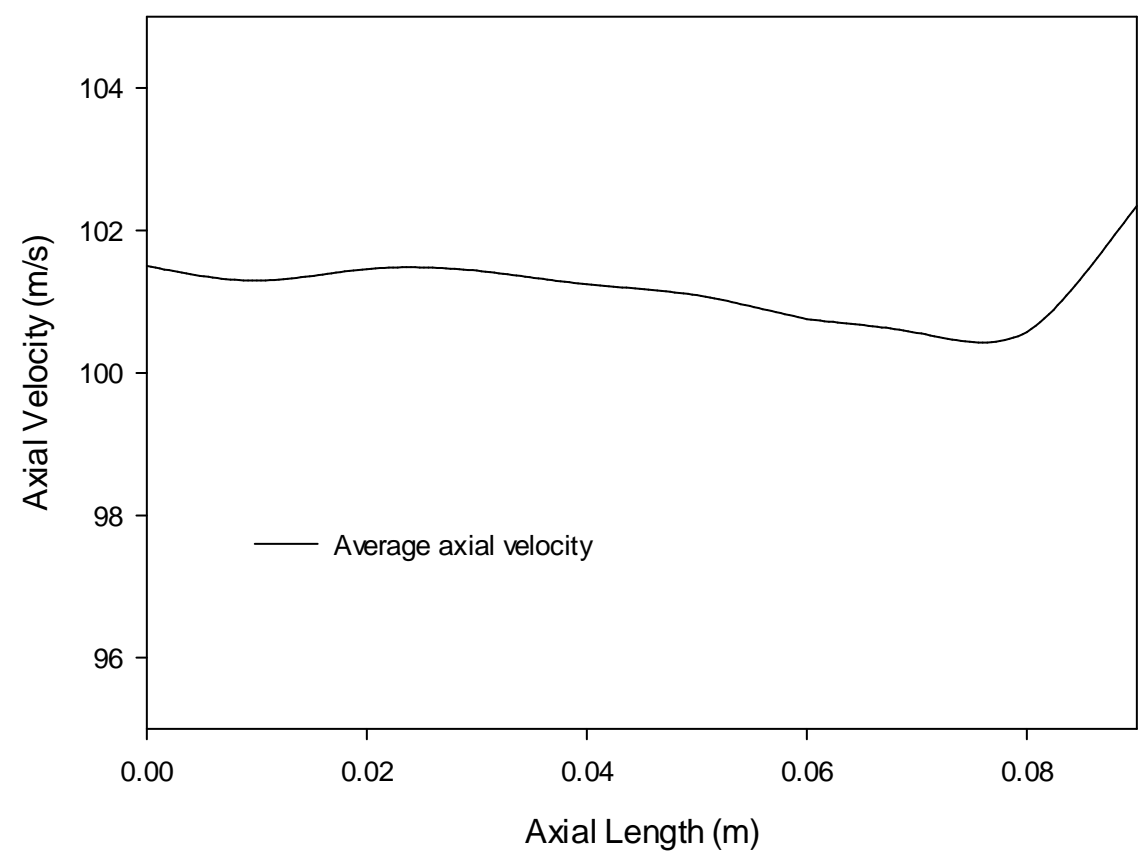

Figure 15: Case 1 - Average Axial Velocity Profile 


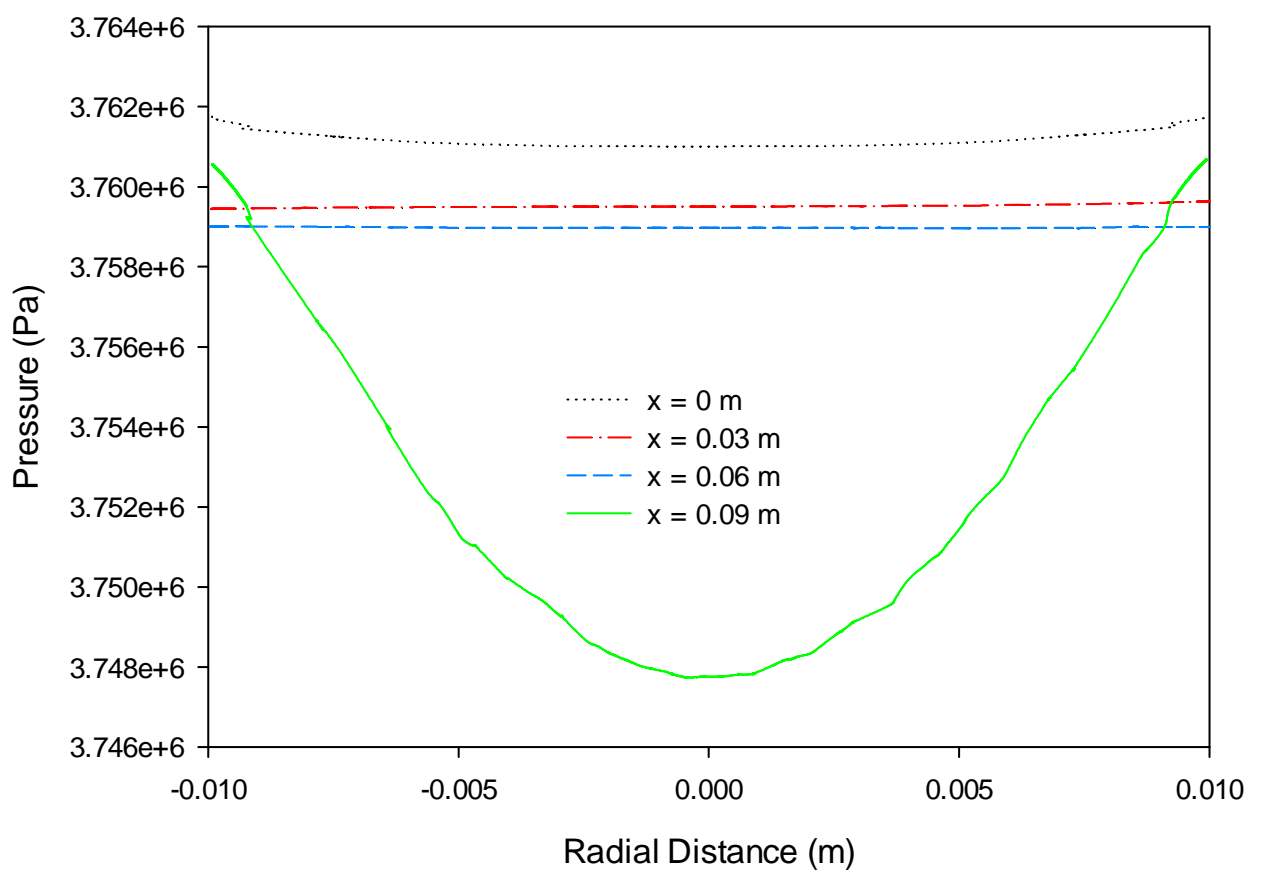

Figure 16: Case 1 - Detailed Pressure Profiles

Table 3: Case 1 - Inlet Conditions

\section{Head-End Inlet Conditions}

\begin{tabular}{ll}
\hline Mass flow rate $(\mathrm{kg} / \mathrm{s})$ & 0.12716 \\
Average Pressure $(\mathrm{Pa})$ & $3,761,275$ \\
Axial Velocity $(\mathrm{m} / \mathrm{s})$ & 101.50 \\
Tangential Velocity $(\mathrm{m} / \mathrm{s})$ & 0 \\
\hline
\end{tabular}




\section{$\underline{\text { Case 2: Weak Swirl Flow Without Transpiration }}$}

The second case for the simulations is a weak swirl flow in the engine, where the swirl number is equal to 0.385 . Equivalently, this swirl flow can be generated by an outward-oriented swirl injector with the swirl angle at $30^{\circ}$. The head-end inlet stipulated flow profile is that consistent with a plug flow representation, whereby one has uniform velocity profiles in both axial and tangential directions at the head-end inlet. Again, one should note that the inlet flow profile in this simulation is different from the flow profile assumed by the regression rate model shown in Fig. 4, where the inlet mass is injected from the guide-vanes, producing a tangential velocity profile that enters the fuel grain port with a boundary thickness $\delta$ at both the exterior and interior zones of the maximum tangential velocity. Nevertheless, the constant tangential velocity profile (with the same swirl intensity) used in this case minimizes the complexity of the flow since any changes in the tangential velocity can be detected easily. Also, since the internal ballistic performance of swirl flows in HRE is often associated with the swirl intensity, with less emphasis on the specific type of injectors, using a basic type of injection, providing a constant tangential velocity, was considered a good reference arrangement for this short simulation study. For the current case, the simulation results are displayed in Fig. 17 showing the streamline profile, pressure contour profile, axial velocity contour profile, and tangential velocity contour profile. Additional detailed results of the pressure and velocity profiles are provided in Figs. 18 to 22 .

From the results, pressure is higher near the wall and lower towards the center due to the centrifugal acceleration caused by the swirl flow. Furthermore, centrifugal force distributes a higher mass flow rate around the outer region, such that a higher axial velocity can be observed. The effect of wall friction and shear flow can be seen, where both axial and tangential velocity are extremely low near the wall, and they both increase moving closer to the central flow. Overall, the average tangential velocity, which is measured from the entire cross-sectional surface perpendicular to the axial flow at various locations, decreases as the flow travels downstream along the fuel grain port. This dissipation of the average tangential velocity may be attributed in part to losses one associates with viscous flow (results obtained later in this section, for inviscid flow, showed decay as well, but at a nominally lower level, perhaps lending some 
support to this presumption). Additionally, it is important to note that the center of the flow rotation should be a singular point; however, this is not properly presented, as shown in Fig. 20, due to the rather coarse mesh of the computational nodes. Similar results are also observed in the later cases.

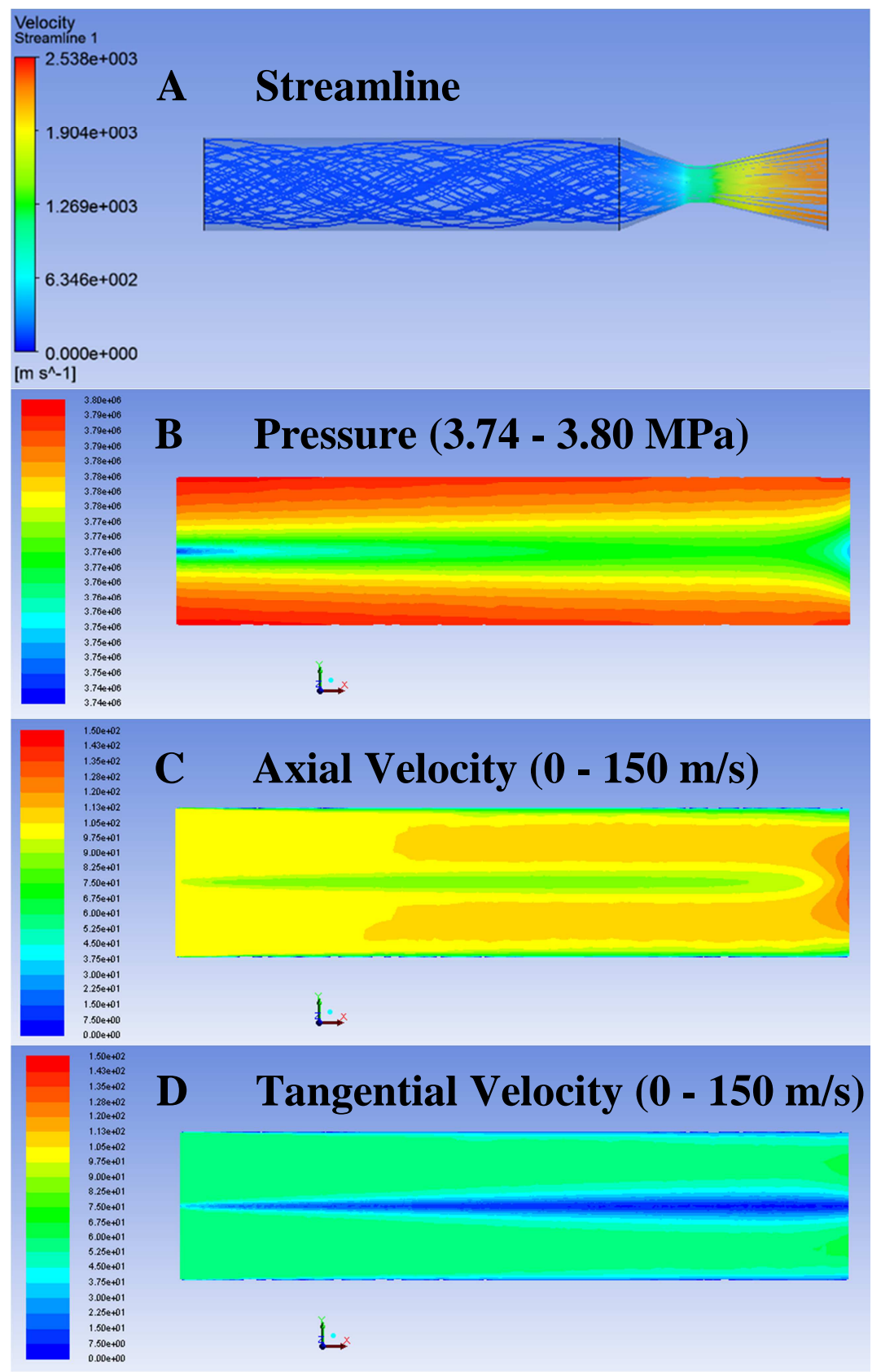

Figure 17: Case 2 - Streamlines, Pressure, Axial Velocity, and Tangential Velocity 


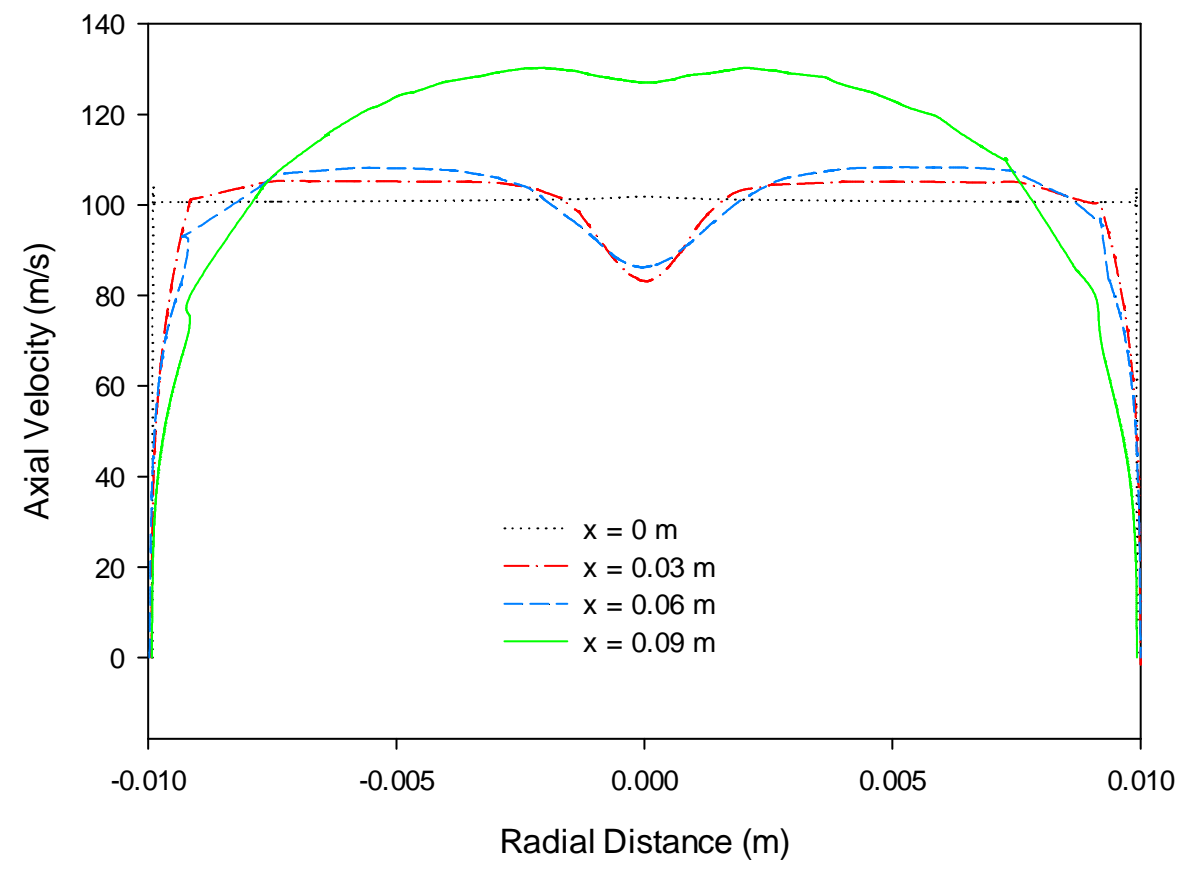

Figure 18: Case 2 - Detailed Axial Velocity Profiles

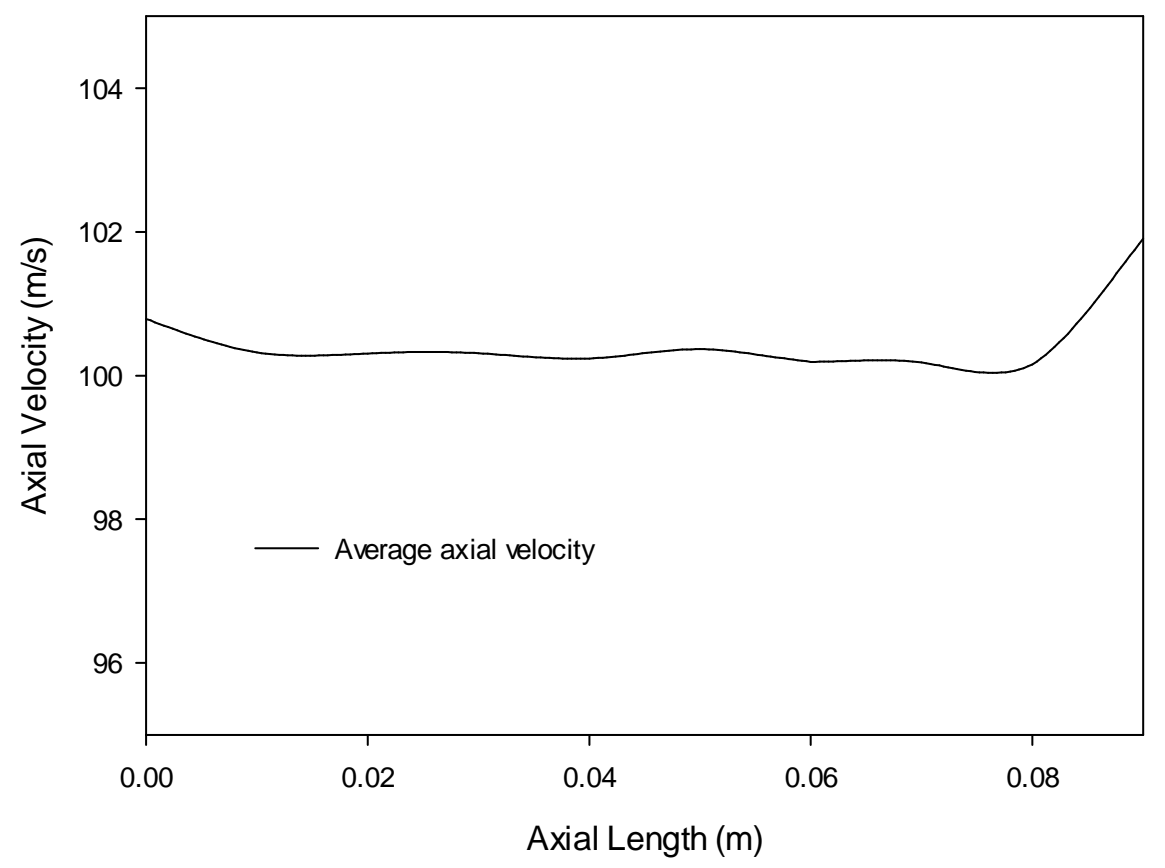

Figure 19: Case 2 - Average Axial Velocity Profile 


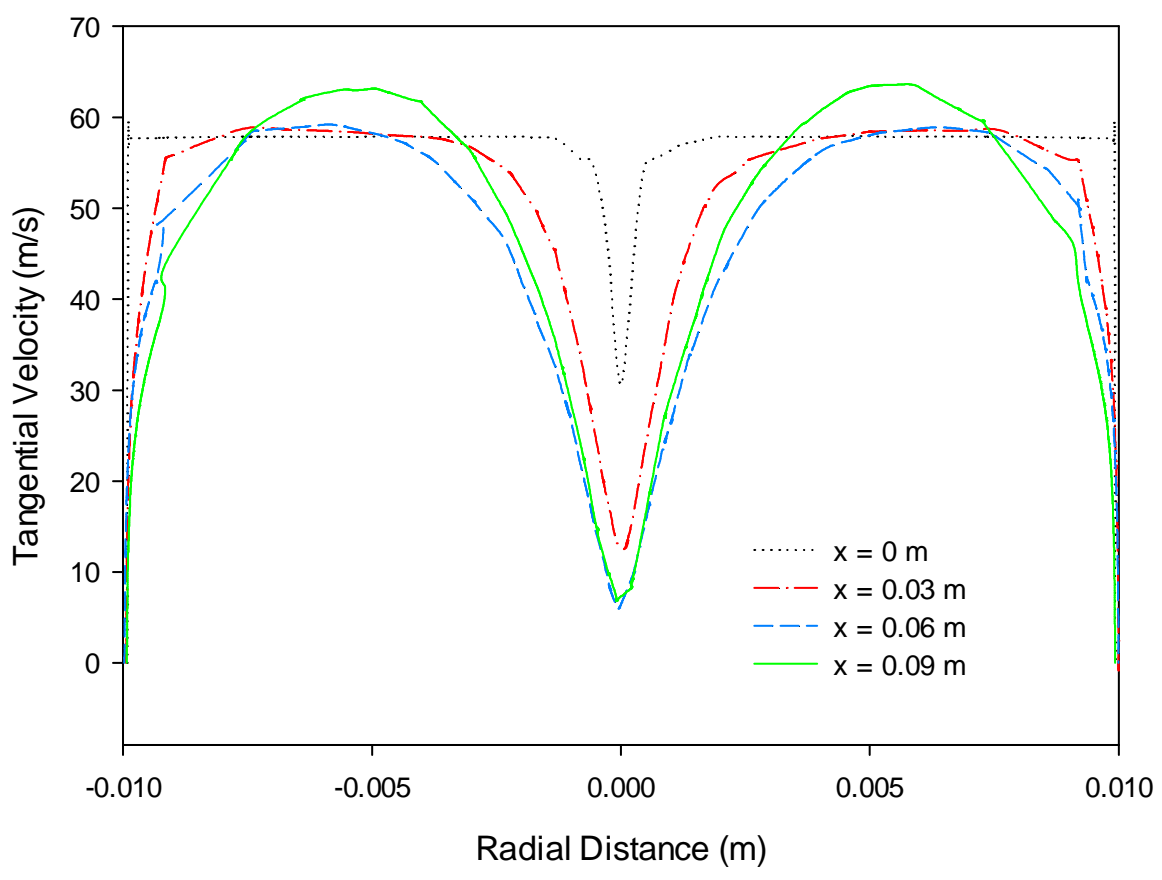

Figure 20: Case 2 - Detailed Tangential Velocity Profiles

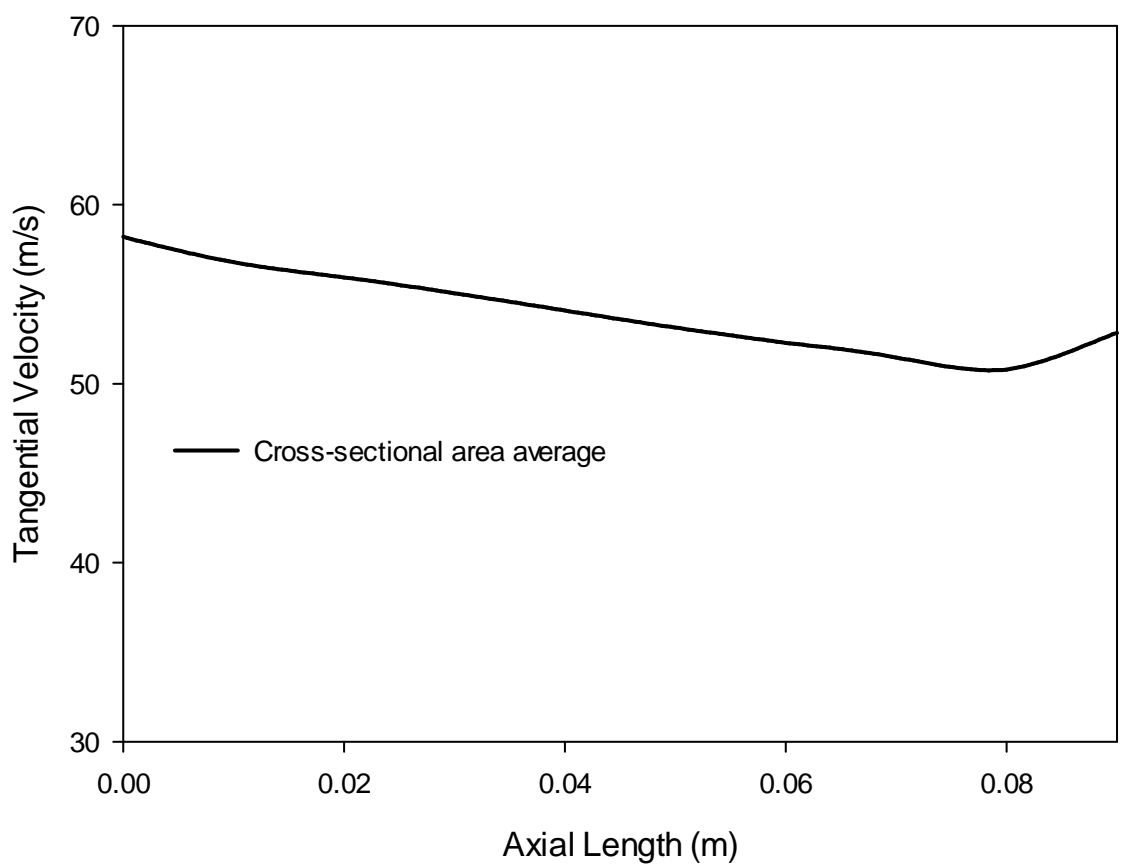

Figure 21: Case 2 - Average Tangential Velocity Profile 


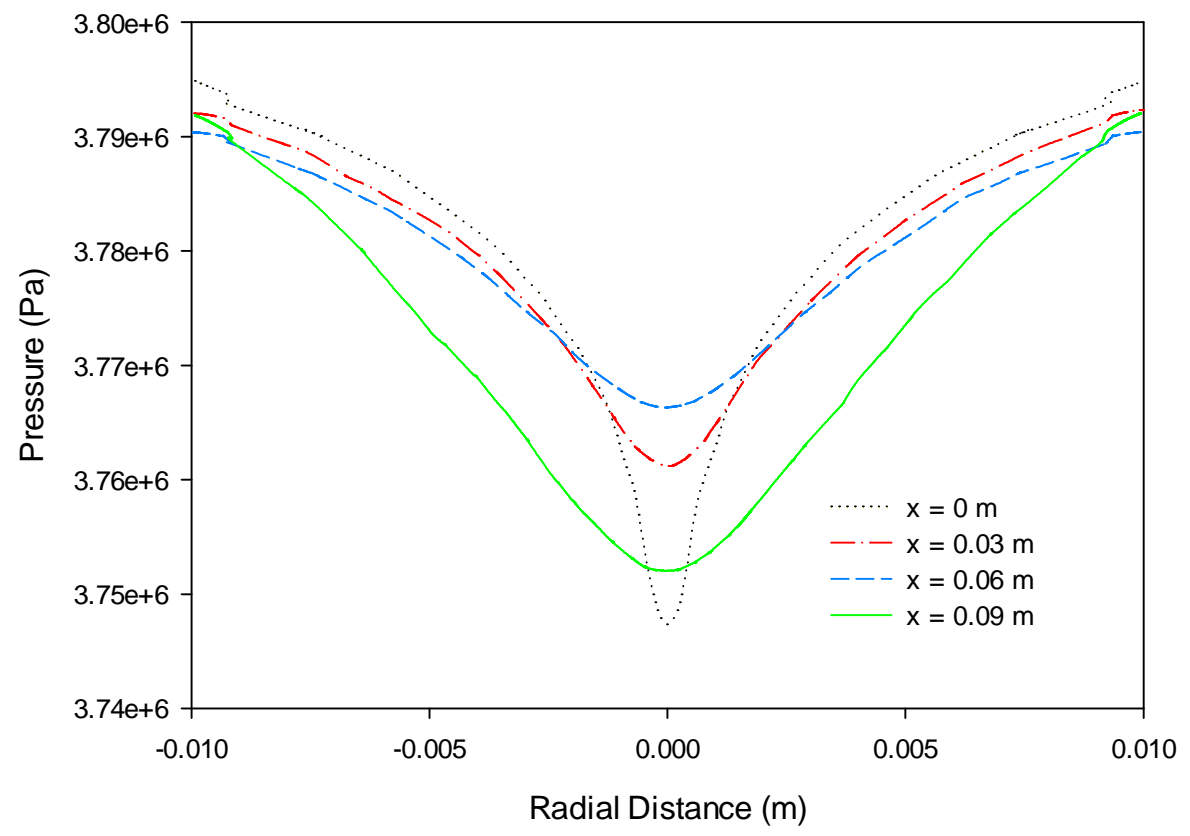

Figure 22: Case 2 - Detailed Pressure Profiles

Table 4: Case 2 - Inlet Conditions

\begin{tabular}{ll}
\hline \multicolumn{2}{c}{ Head-End Inlet Conditions } \\
\hline Mass flow rate $(\mathrm{kg} / \mathrm{s})$ & 0.12716 \\
Average Pressure $(\mathrm{Pa})$ & $3,787,563$ \\
Axial Velocity $(\mathrm{m} / \mathrm{s})$ & 100.78 \\
Tangential Velocity $(\mathrm{m} / \mathrm{s})$ & 58.20 \\
\hline
\end{tabular}




\section{Case 3: Strong Swirl Flow Without Transpiration}

Another simulation for better understanding the behavior of swirl flows in HREs is undertaken, with flow from the head-end inlet now having a swirl number of 0.667 , which is a strong swirl flow, as defined previously. This swirl number corresponds to a head-end injector with a swirl angle of $45^{\circ}$. The simulation results displayed in Fig. 23 show the streamline profile, pressure contour profile, axial velocity contour profile, and tangential velocity contour profile. Additional detailed results of the pressure and velocity profiles are provided in Figs. 24 to 28.

Similar effects on pressure, axial velocity, and tangential velocity can be observed, as seen earlier for the weak swirl case. However, the effects of the swirl flow on pressure and velocity profiles are more pronounced in this case. As shown in Fig. 28, there is a larger difference between the pressure around the wall and the center of the flow. Since the swirl intensity is considerably strong in this case, the pressure difference near the head-end inlet becomes high enough to move the flow axially backwards, as shown Fig. 23 (c), where axial velocity becomes negative. This so-called recirculation zone, which appears in the head-end region, helps confirm that the simulation model properly accounts for the factors affecting the flow behavior, at least with some accuracy. Some other effects, such as the near wall profile caused by wall friction and the apparent decay of the averaged tangential velocity, can still be observed in this study.

As a side note, ideally the axial velocity profiles in Fig. 24 should have the same areas under the curves for the region far away from the nozzle, e.g., at $\mathrm{x}=0.03$ and $\mathrm{x}=0.06$, in order to sustain the conservation of mass, since there is no significant change of density in this region. However, possible errors could occur since the presented data is measured based on a single line drawn across the flow cross-sectional area.

All the baseline swirl effects (no transpiration present) have been looked at in Cases 2 and 3. It is hoped that the information obtained will provide further help towards understanding more complex situations involving swirl, e.g., when transpiration is being incorporated. 


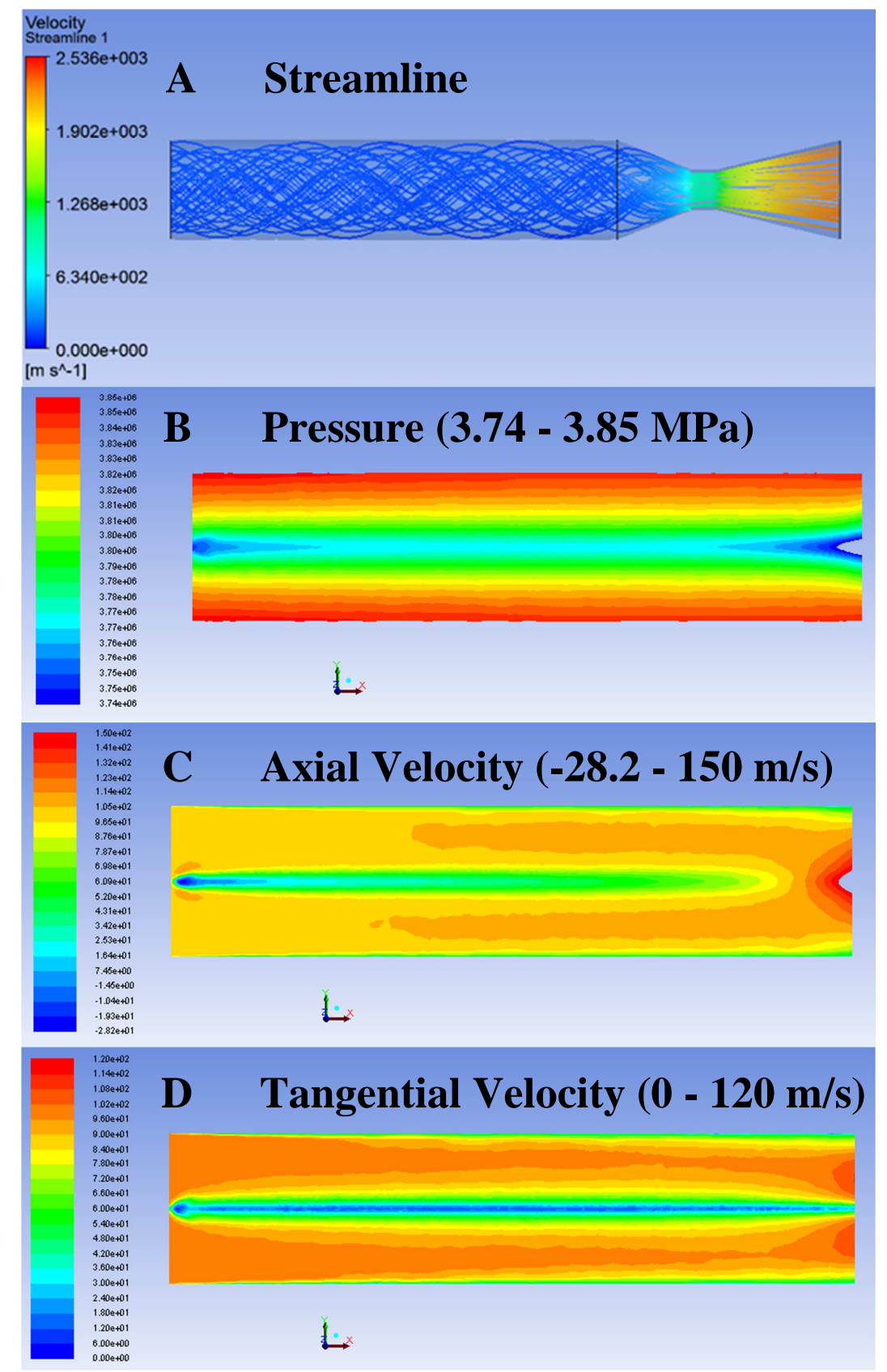

Figure 23: Case 3 - Streamlines, Pressure, Axial Velocity, and Tangential Velocity 


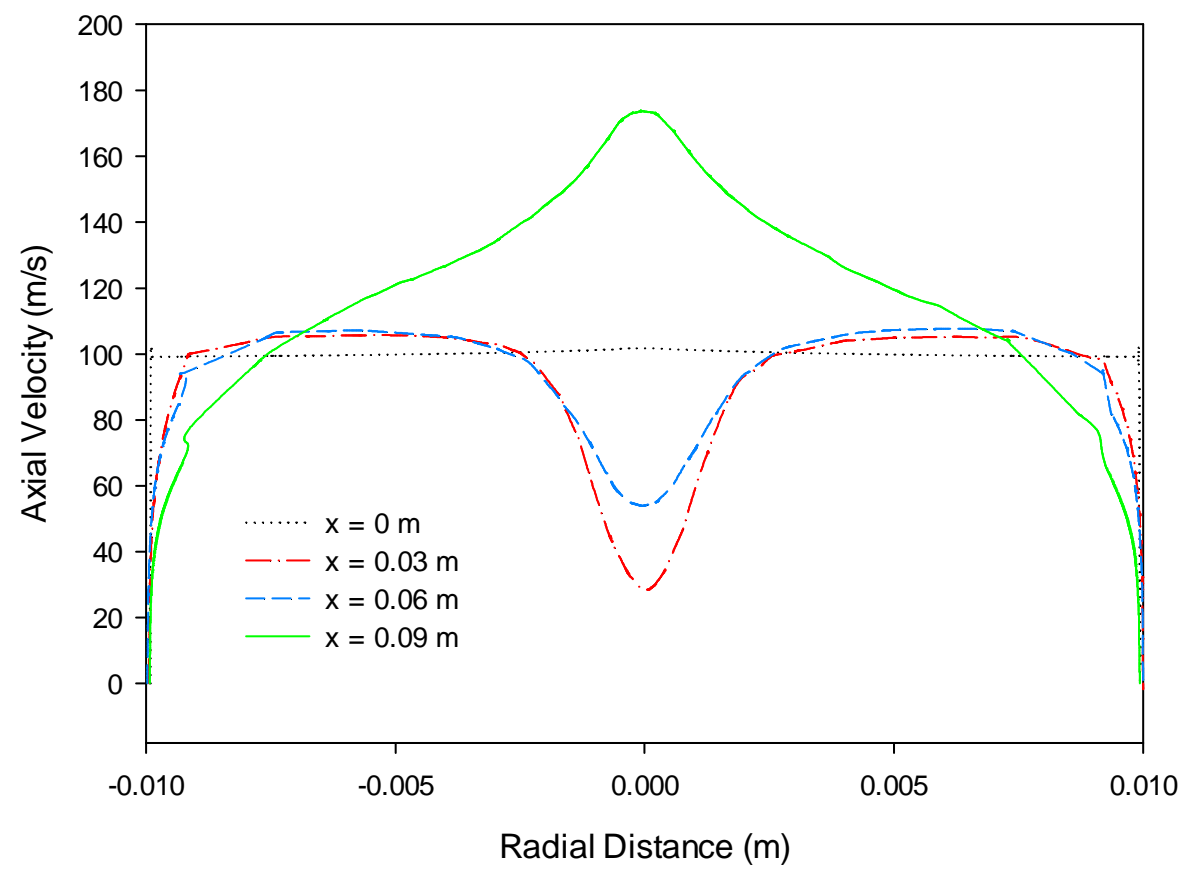

Figure 24: Case 3 - Detailed Axial Velocity Profiles

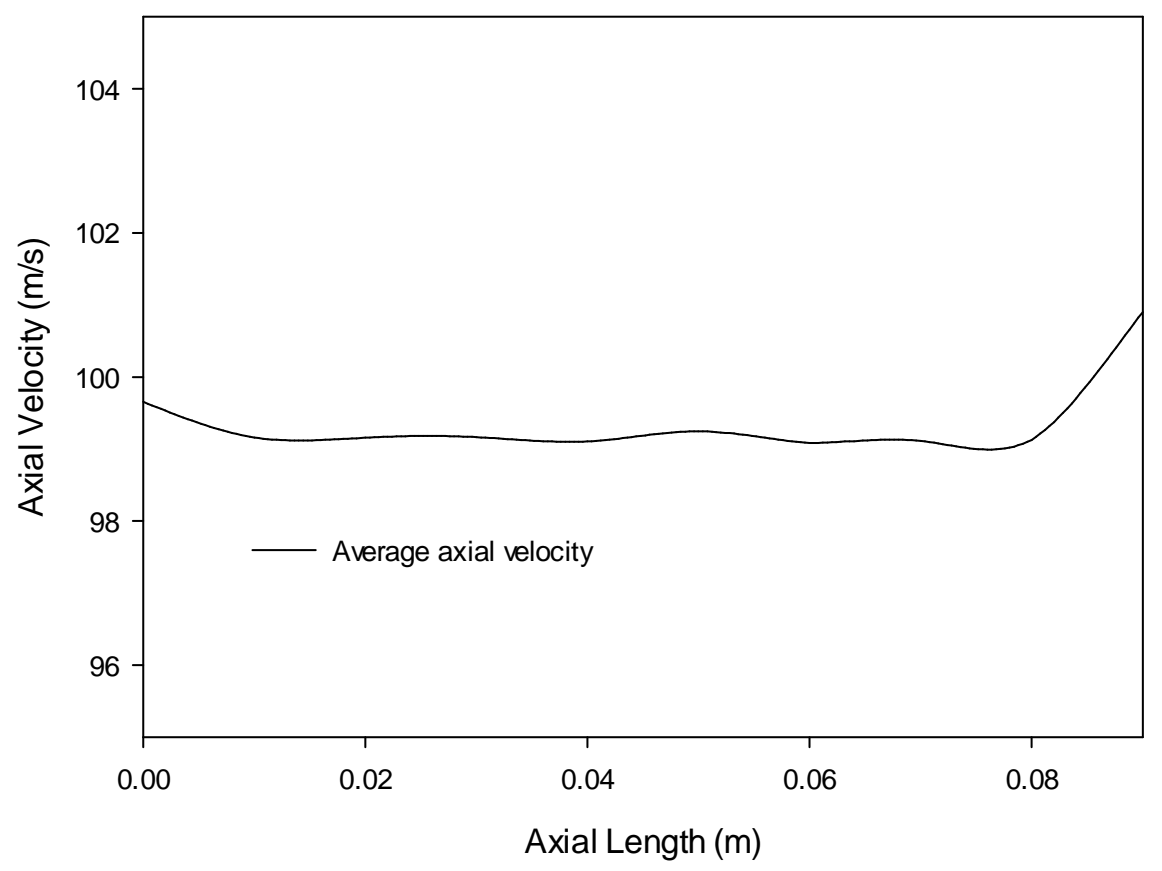

Figure 25: Case 3 - Average Axial Velocity 


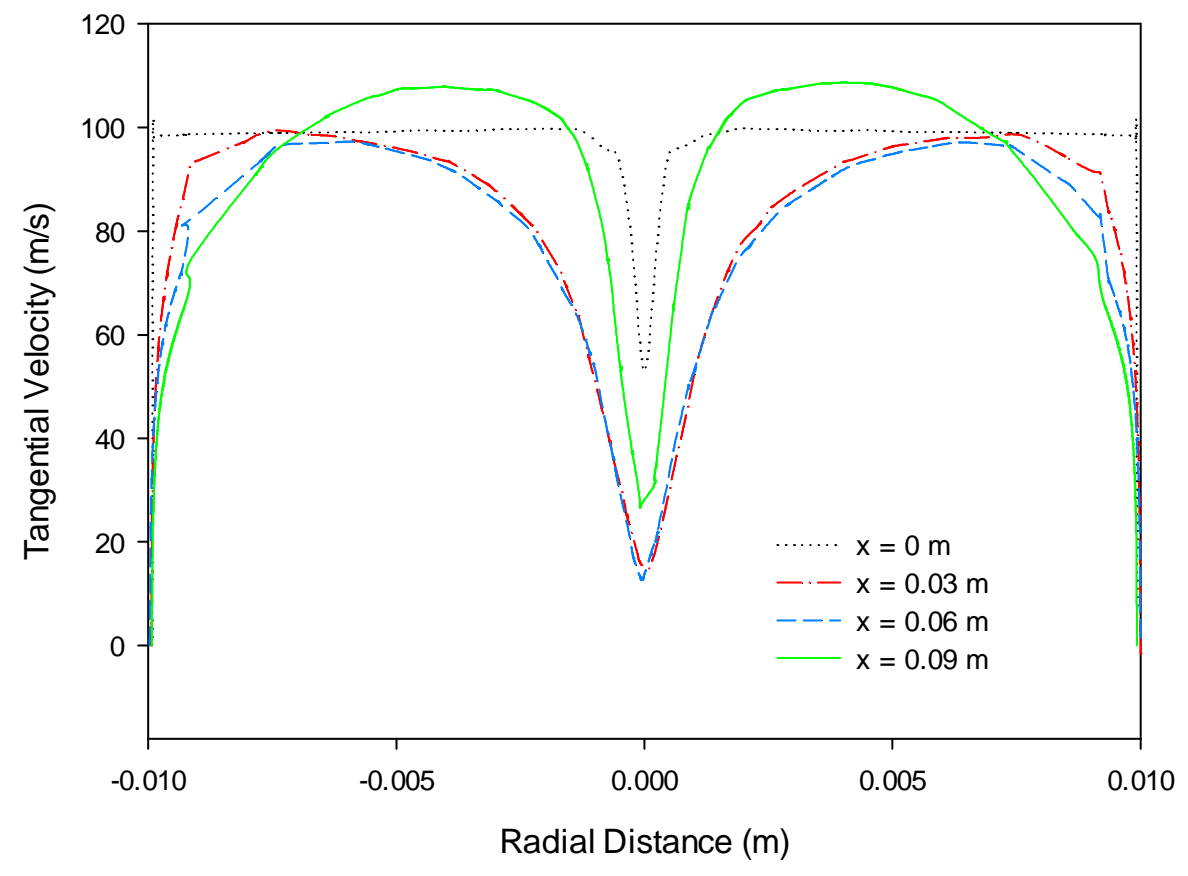

Figure 26: Case 3 - Detailed Tangential Velocity Profiles

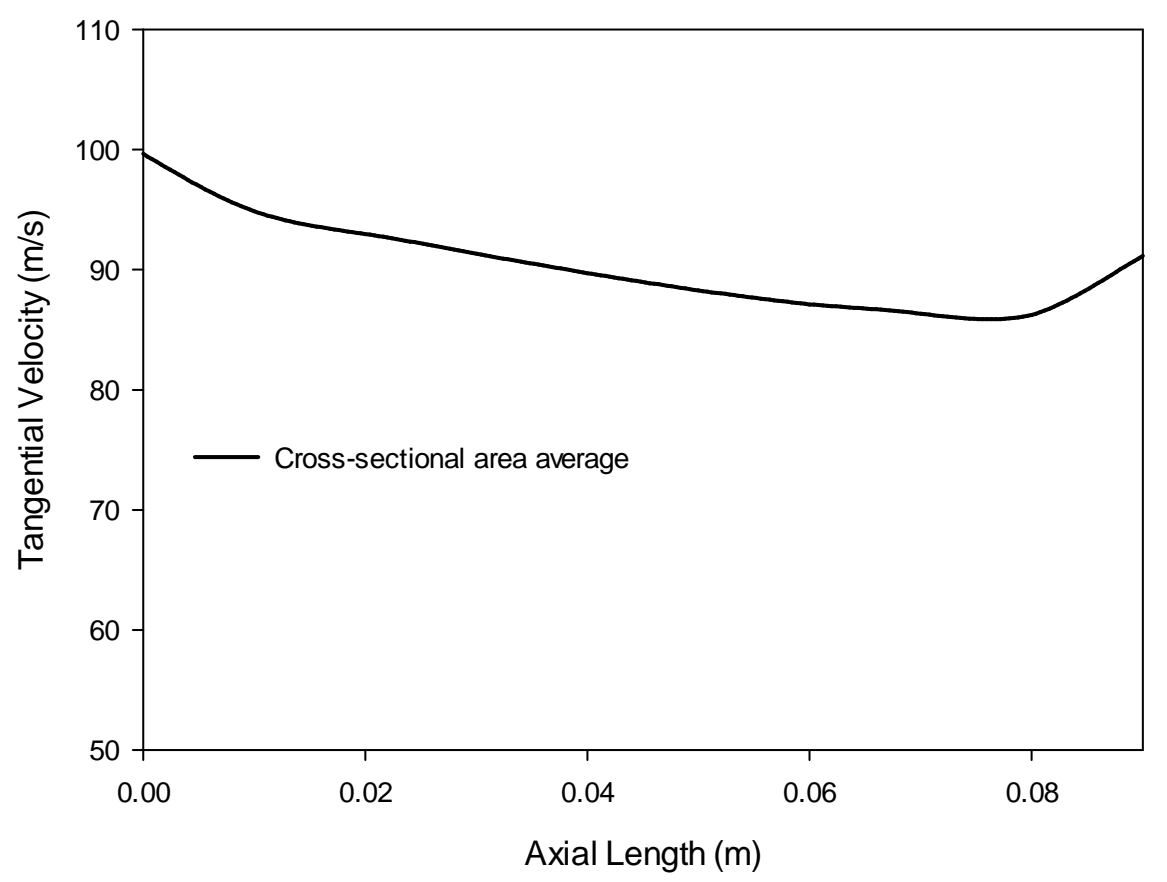

Figure 27: Case 3 - Average Tangential Velocity 


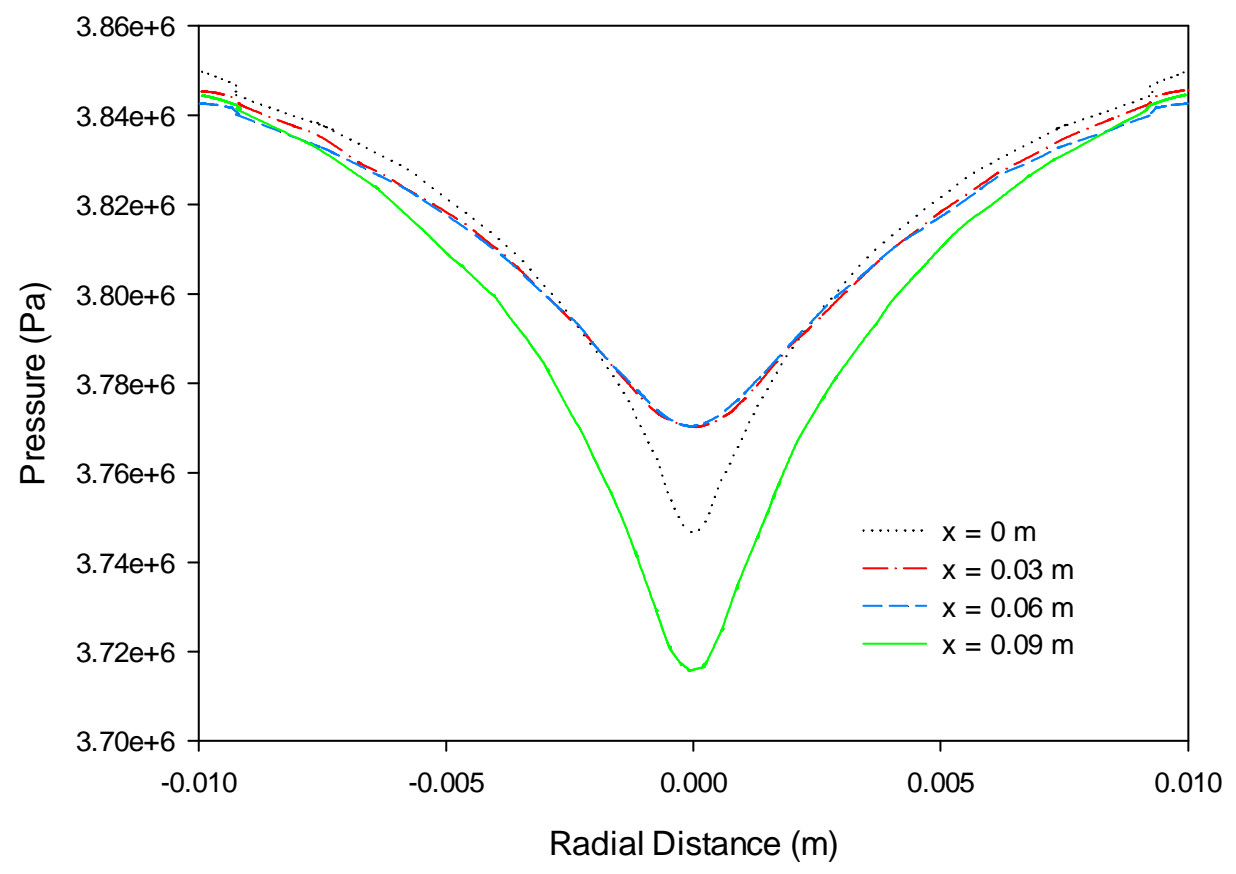

Figure 28: Case 3 - Detailed Pressure Profiles

Table 5: Case 3 - Inlet Conditions

\begin{tabular}{ll}
\hline \multicolumn{2}{c}{ Head-End Inlet Conditions } \\
\hline Mass flow rate $(\mathrm{kg} / \mathrm{s})$ & 0.12716 \\
Average Pressure $(\mathrm{Pa})$ & $3,829,746$ \\
Axial Velocity $(\mathrm{m} / \mathrm{s})$ & 99.65 \\
Tangential Velocity $(\mathrm{m} / \mathrm{s})$ & 99.65 \\
\hline
\end{tabular}




\subsection{Full Model Simulation}

In the case of the full engine model (with transpiration) being simulated, the mass flow rate of the gas entering at the head-end inlet is $0.09716 \mathrm{~kg} / \mathrm{s}$, while the mass flow rate entering radially from the fuel grain surface is $0.03 \mathrm{~kg} / \mathrm{s}$. These mass flow rates are approximated based on the actual experimental data presented in the literature for a comparable engine. ${ }^{21}$ Three schemes of the full model simulation are evaluated, by varying the conditions of the flow as discussed in Case 4, 5, and 6. This includes an axial (non-swirl) viscous flow, a swirling viscous flow with $S$ $=0.385$, and an inviscid swirl flow with $S=0.385$. The choice of modeling inviscid flow (and comparing to the viscous flow case) within the engine will be elaborated upon below.

\section{Case 4: Axial Flow With Transpiration}

The first case considered for a full simulation with transpiration is an axial (non-swirling) viscous flow. With transpiration, the core flow experiences the influence of a constant mass flow rate moving radially into the central core flow along the length solid fuel surface. The volumetric area being monitored is the section designated for the fuel grain surface, which is along the axial length of the engine model between 0.02 to $0.09 \mathrm{~m}$. Both the head-end inlet and the fuel grain surface inject a propellant gas with the properties as listed in Table 1. The results displayed in Fig. 29 show the streamline profile, pressure contour profile, and axial velocity contour profile. Additional detailed results of the pressure and velocity profiles are provided in Figs. 30 to 32.

Unlike the previous cases, the core flow is slightly pushed towards the center as it travels along the axial length, due to the radially-oriented incoming propellant gas flow. The pressure profile shows a decrease as the additional mass is added by transpiration to the central flow as one moves downstream along the fuel grain. With this mass influx, the axial flow correspondingly accelerates as it is moving downstream. Additionally, transpiration lowers the friction experienced by the core flow at the bounding port surface, hence the pressure drop (and corresponding additional acceleration of the flow) due to viscous flow losses should decrease somewhat. ${ }^{10}$ 


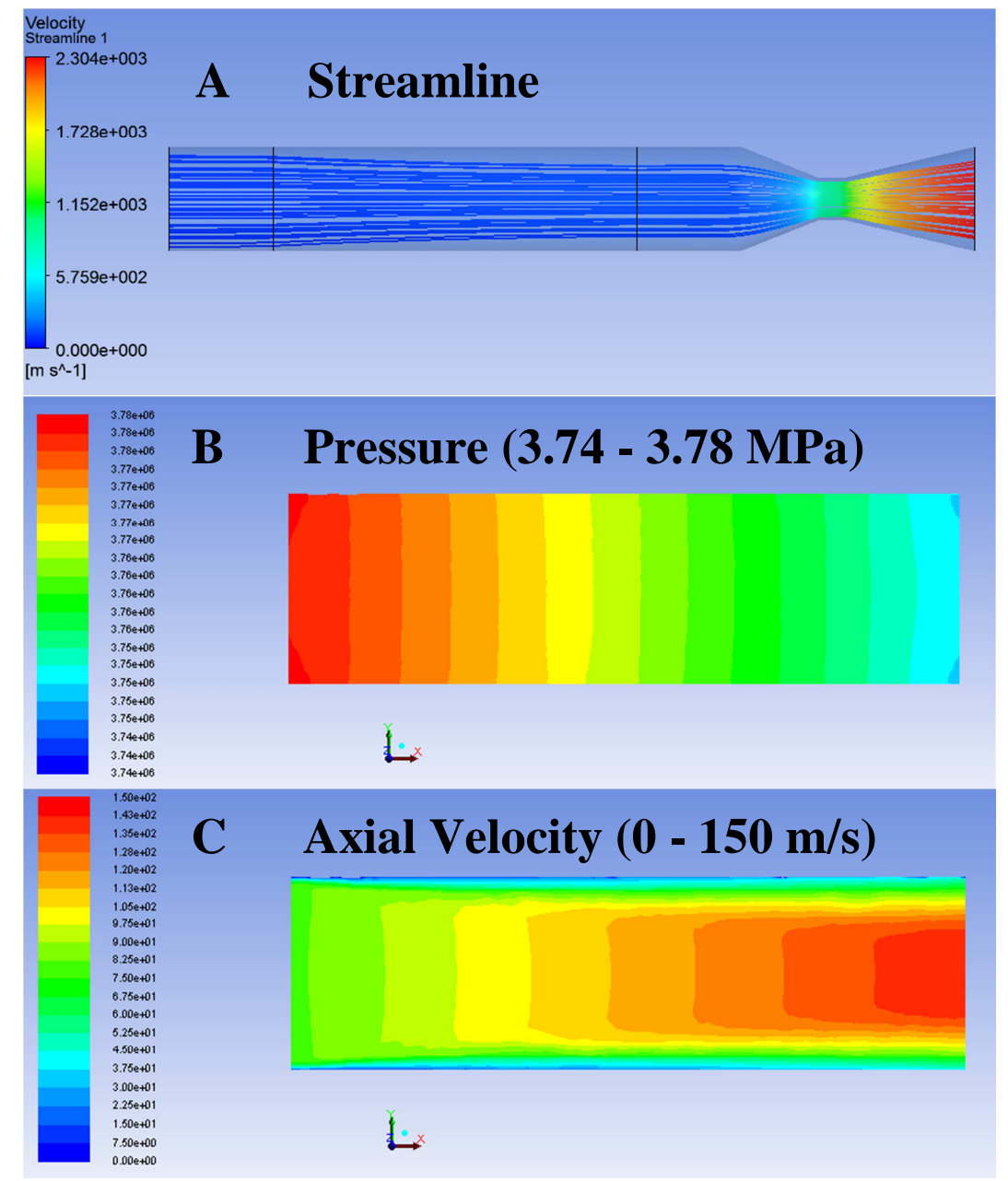

Figure 29: Case 4 - Streamlines, Pressure, and Axial Velocity 


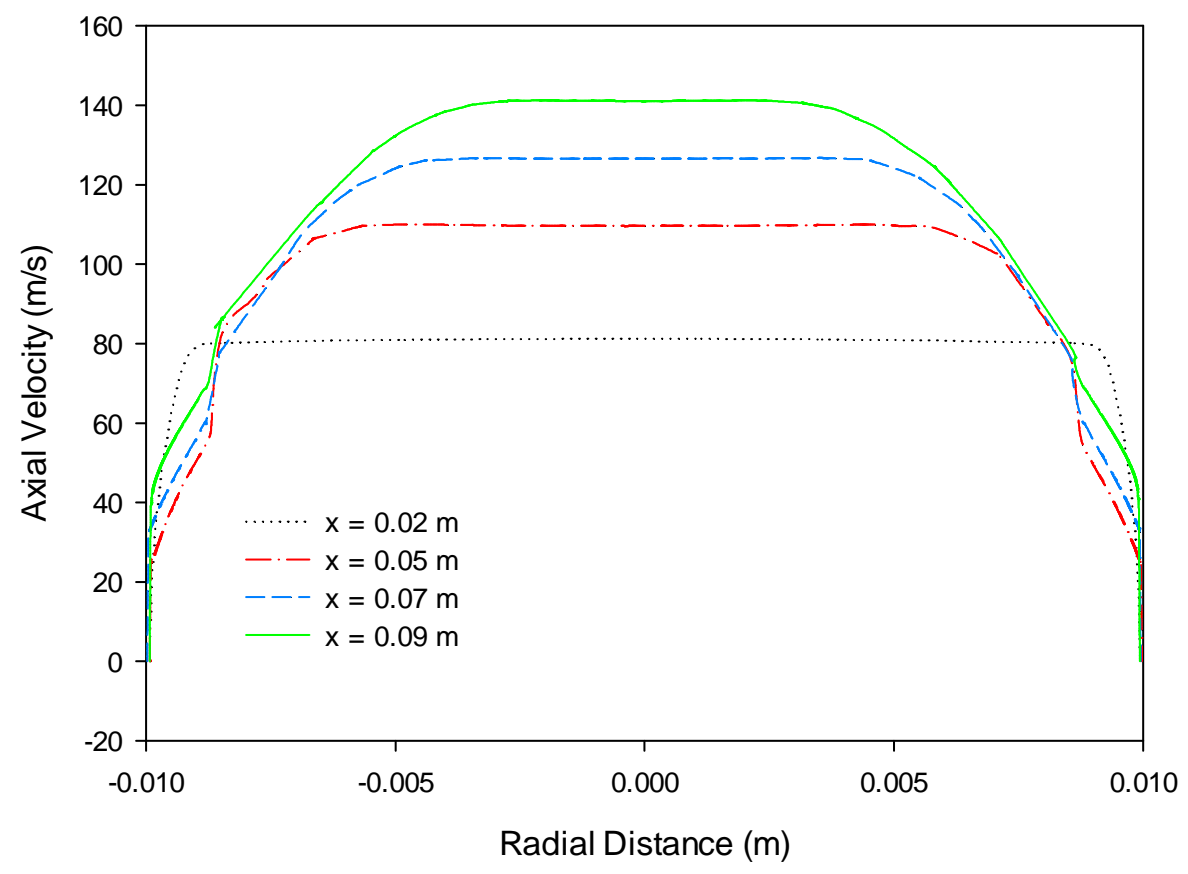

Figure 30: Case 4 - Detailed Axial Velocity Profiles

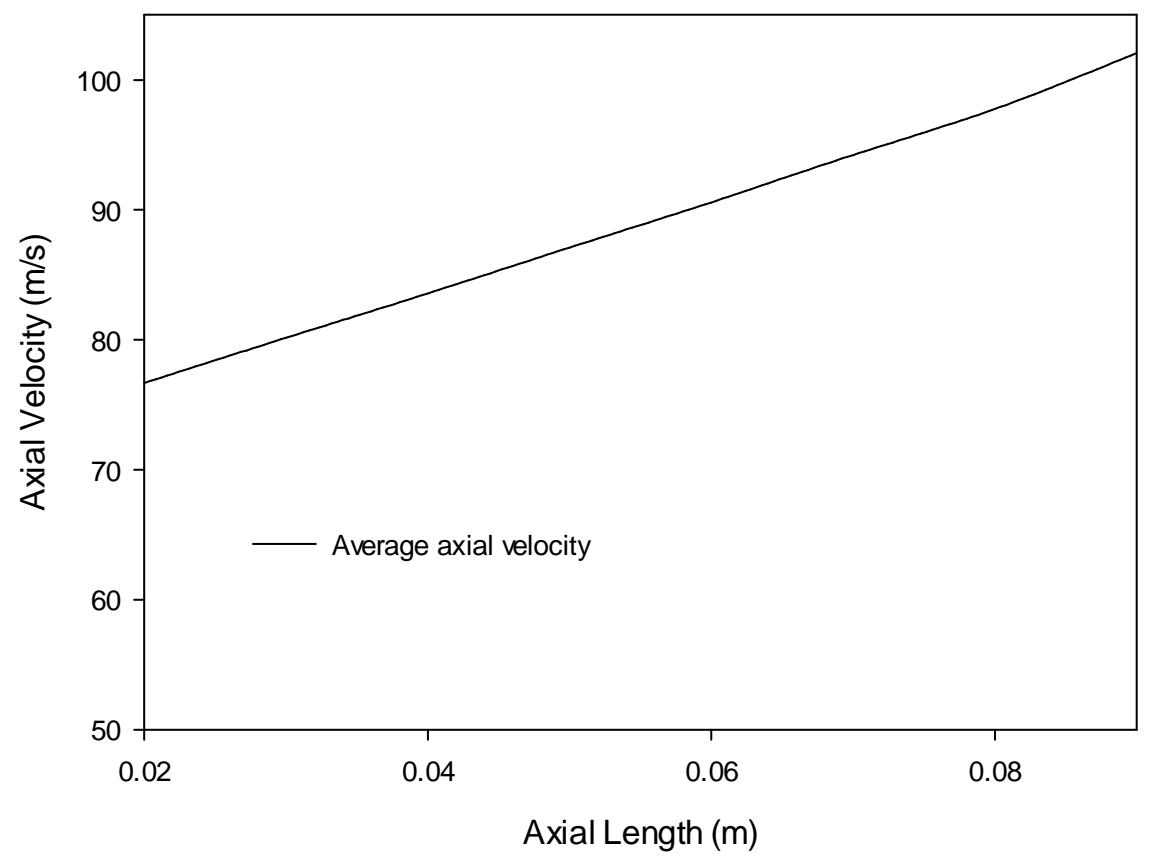

Figure 31: Case 4 - Average Axial Velocity 


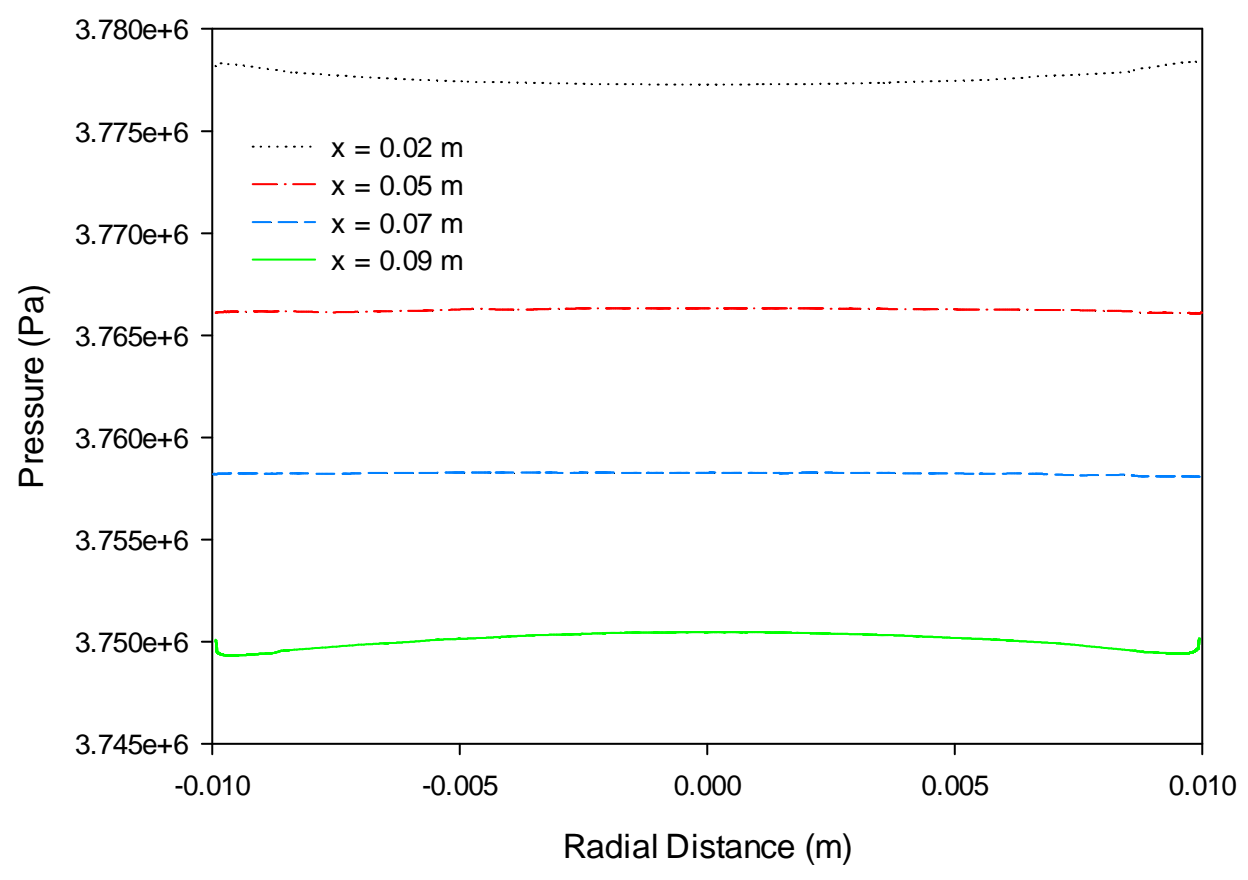

Figure 32: Case 4 - Detailed Pressure Profiles

Table 6: Case 4 - Inlet Conditions

\begin{tabular}{ll}
\hline \multicolumn{2}{c}{ Head-End Inlet Conditions } \\
\hline Mass flow rate $(\mathrm{kg} / \mathrm{s})$ & 0.09716 \\
Average Pressure $(\mathrm{Pa})$ & $3,778,765$ \\
Axial Velocity $(\mathrm{m} / \mathrm{s})$ & 77.23 \\
Tangential Velocity $(\mathrm{m} / \mathrm{s})$ & 0 \\
\hline
\end{tabular}




\section{Case 5: Swirl Flow With Transpiration}

A critical part of the numerical study is a full model simulation of swirl flow with transpiration. This simulation is set up by using a swirling viscous flow with $S=0.385$, which is injected at the head-end inlet. The simulation results displayed in Fig. 33 show the streamline profile, pressure contour profile, and axial velocity contour profile. Additional detailed results of the pressure and velocity profiles are provided in Figs. 34 to 39.

Similarly as the previous case, the core flow is pushed slightly inward by the radial transpiration mass entering from the fuel grain surface. Together with reducing wall friction, the core flow accelerates, both in the axial and tangential directions. Having the effects of swirl and transpiration combined, the new pressure and axial velocity profiles can be observed. While tangential velocity of a significant portion of the core flow accelerates in the first half of the engine, presumably due to transpiration, the tangential velocity of that flow decays over the latter half of the engine, as may be viewed in Fig. 33 (d). The average tangential velocity consistently decreases from the engine head end, as one moves downstream along the length of the fuel grain.

Looking at Fig. 36, the tangential velocity has peak values at various locations within the core flow. Taking the average of all the peak tangential velocity values along the axial location shown in Fig. 39, the value is found to be $47.43 \mathrm{~m} / \mathrm{s}$. This value is relatively close to the tangential velocity measured at the head-end inlet, which is slightly lower at $44.48 \mathrm{~m} / \mathrm{s}$. 


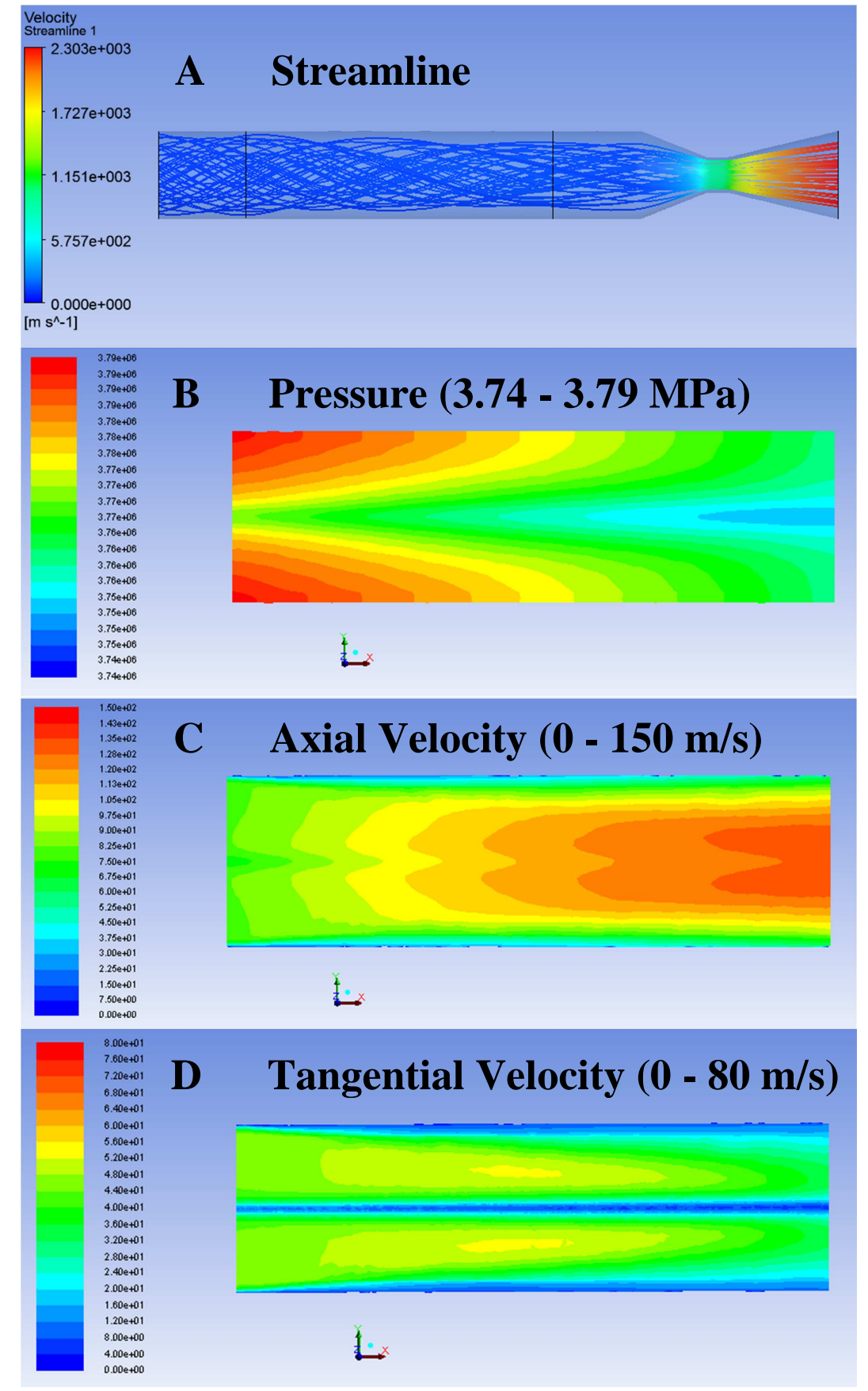

Figure 33: Case 5 - Streamlines, Pressure, Axial Velocity, and Tangential Velocity 


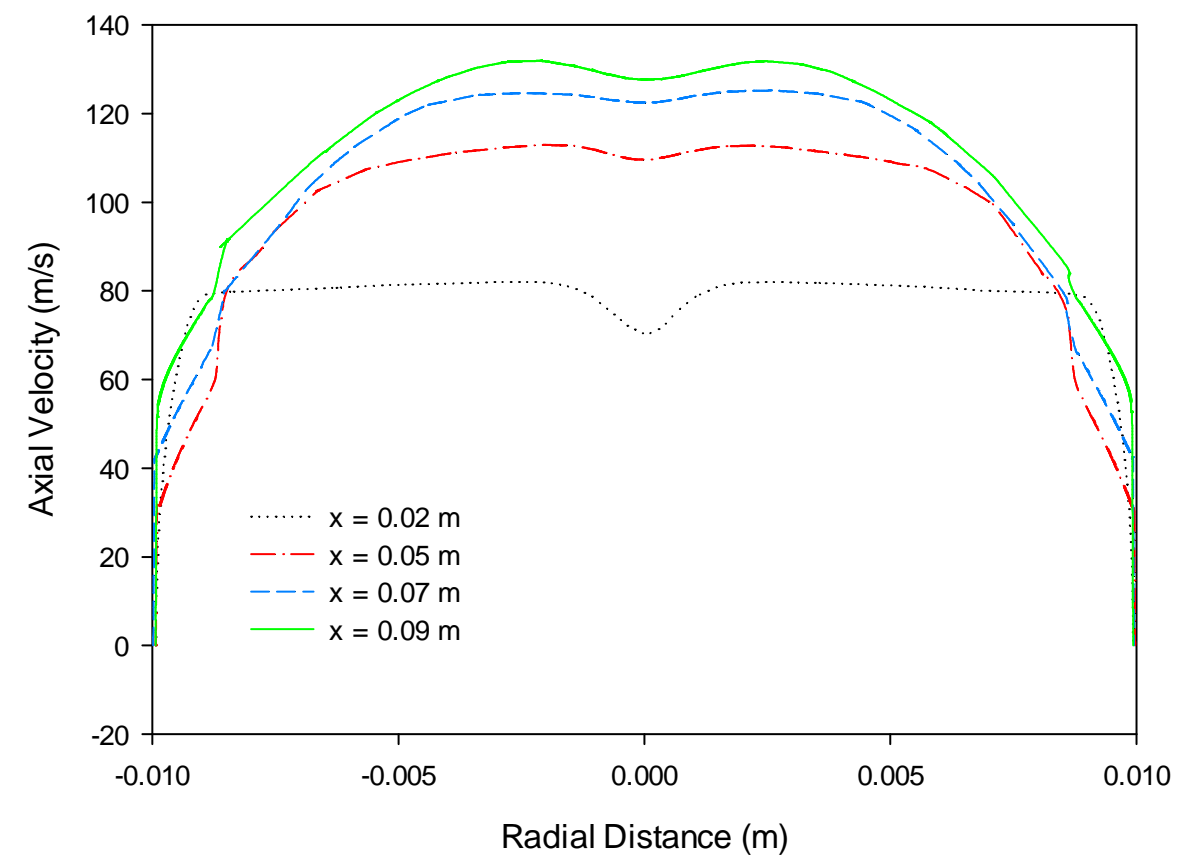

Figure 34: Case 5 - Detailed Axial Velocity Profiles

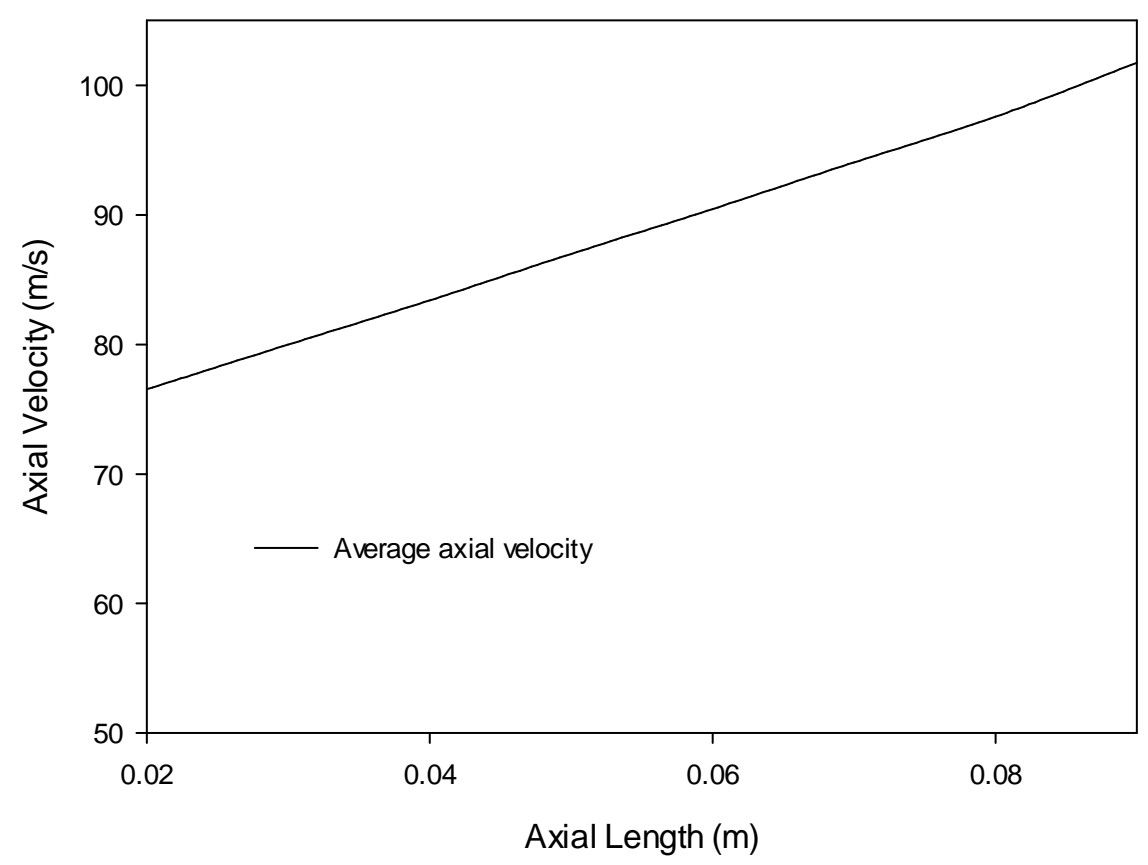

Figure 35: Case 5 - Average Axial Velocity 


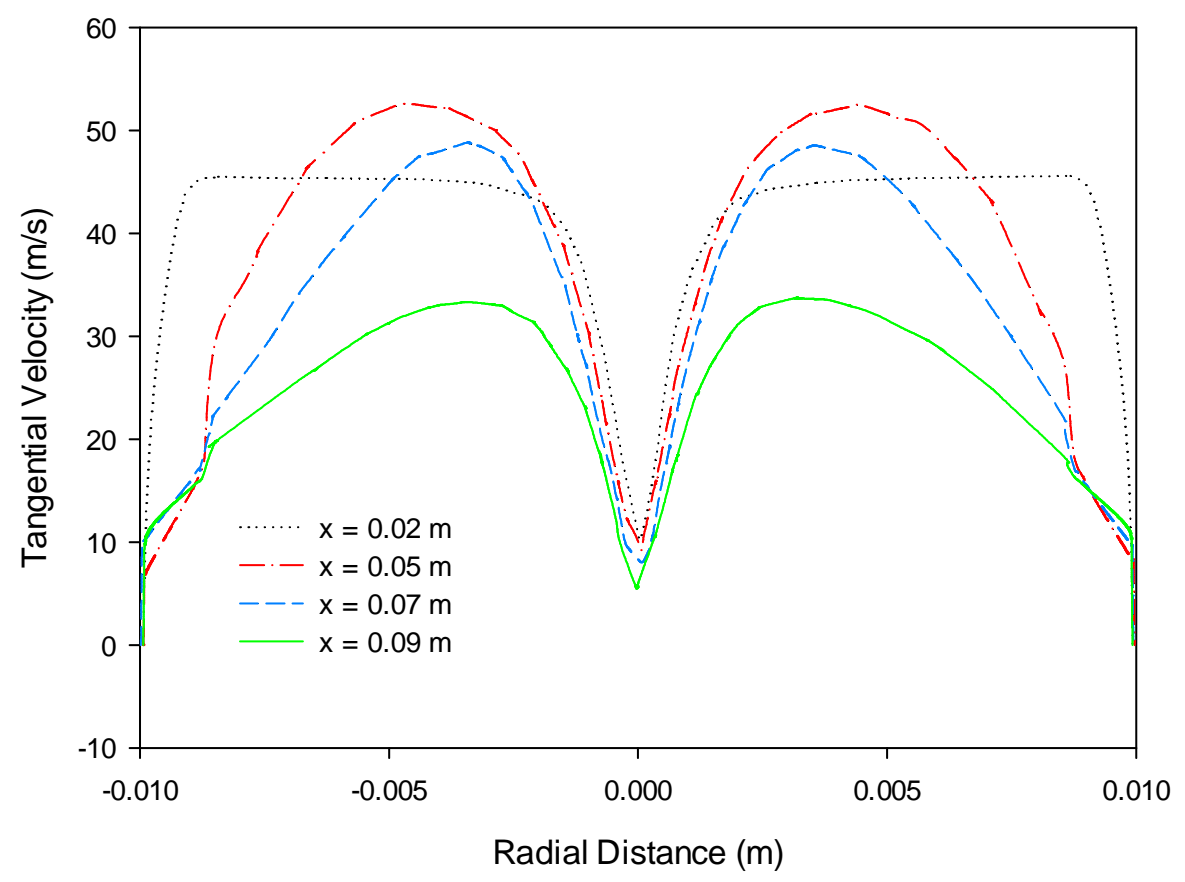

Figure 36: Case 5 - Detailed Tangential Velocity Profiles

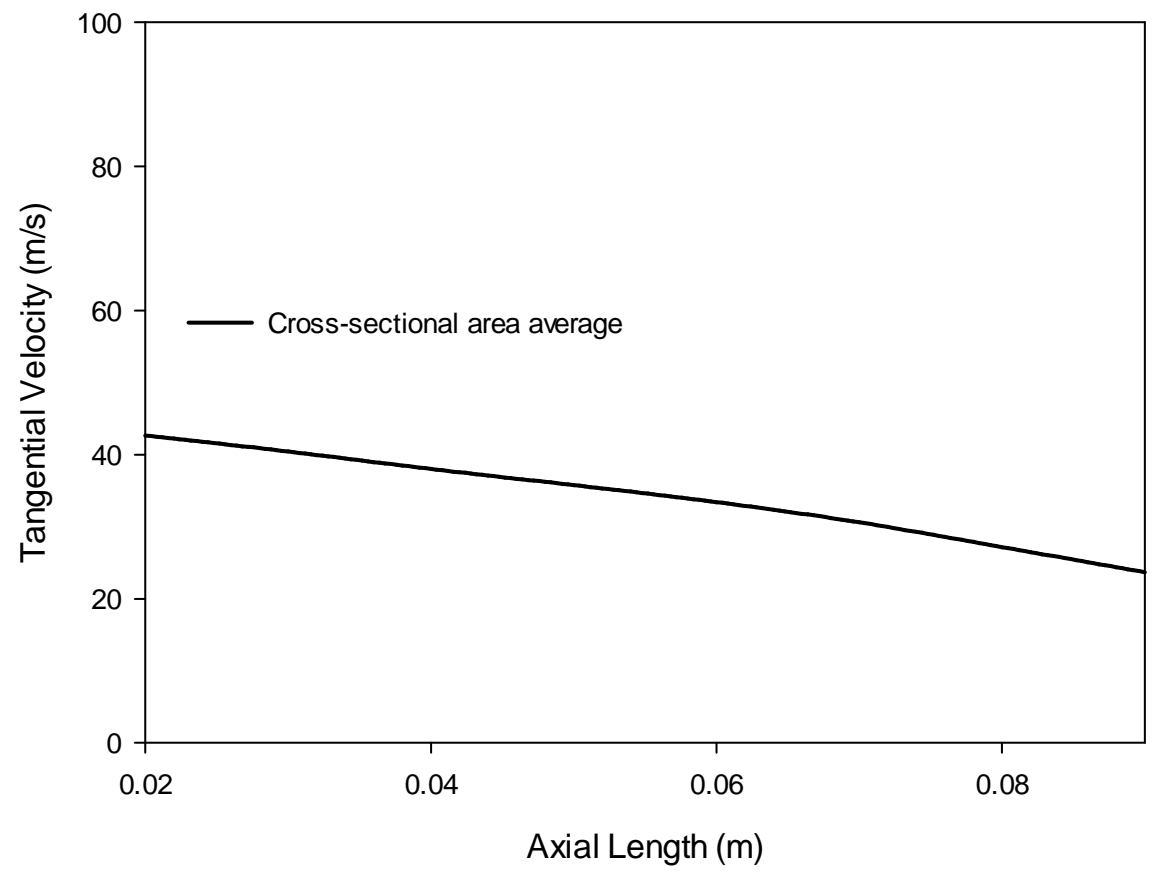

Figure 37: Case 5 - Average Tangential Velocity 


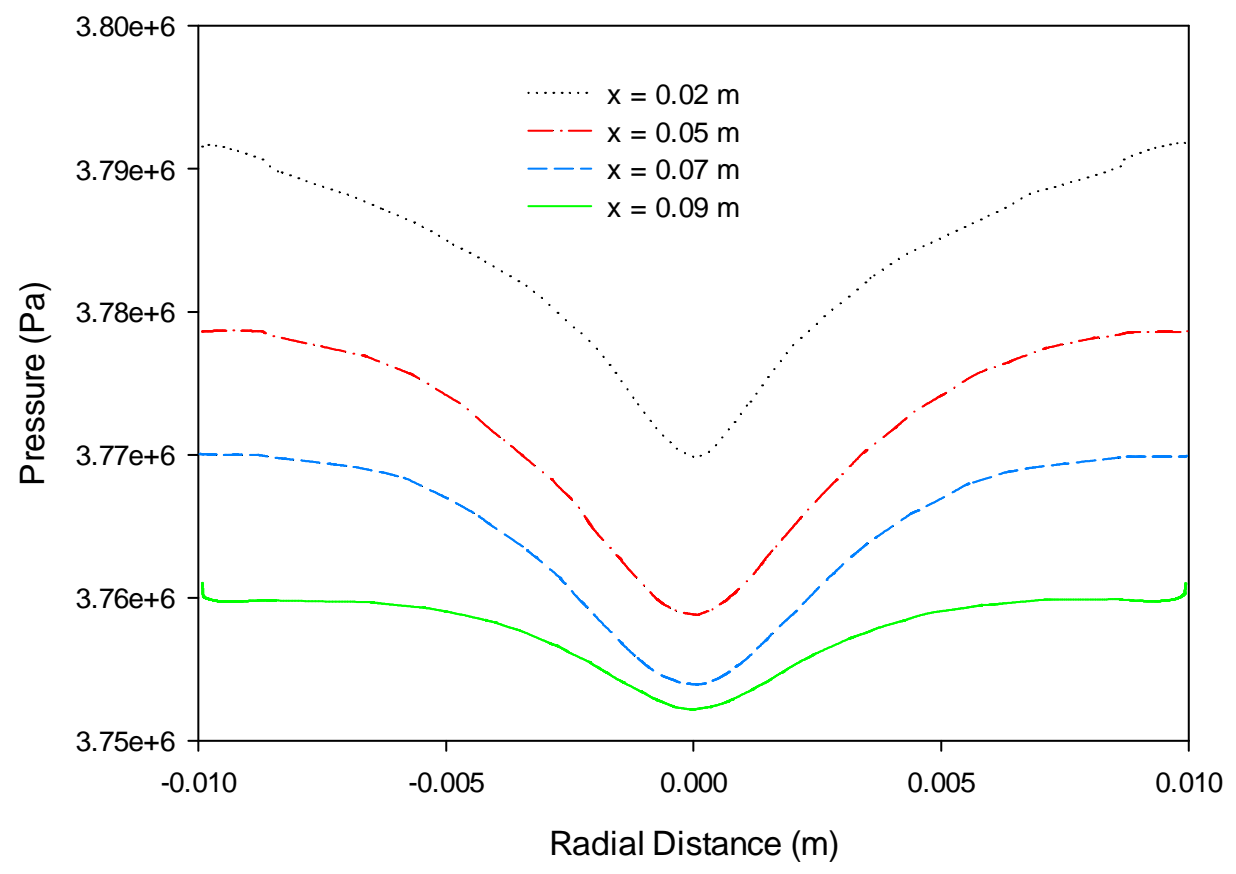

Figure 38: Case 5 - Detailed Pressure Profiles

Table 7: Case 5 - Inlet Conditions

Head-End Inlet Conditions

\begin{tabular}{ll}
\hline Mass flow rate $(\mathrm{kg} / \mathrm{s})$ & 0.09716 \\
Average Pressure $(\mathrm{Pa})$ & $3,788,402$ \\
Axial Velocity $(\mathrm{m} / \mathrm{s})$ & 77.03 \\
Tangential Velocity $(\mathrm{m} / \mathrm{s})$ & 44.48 \\
Average Peak Tangential Velocity $(\mathrm{m} / \mathrm{s})$ & 47.43 \\
\hline
\end{tabular}




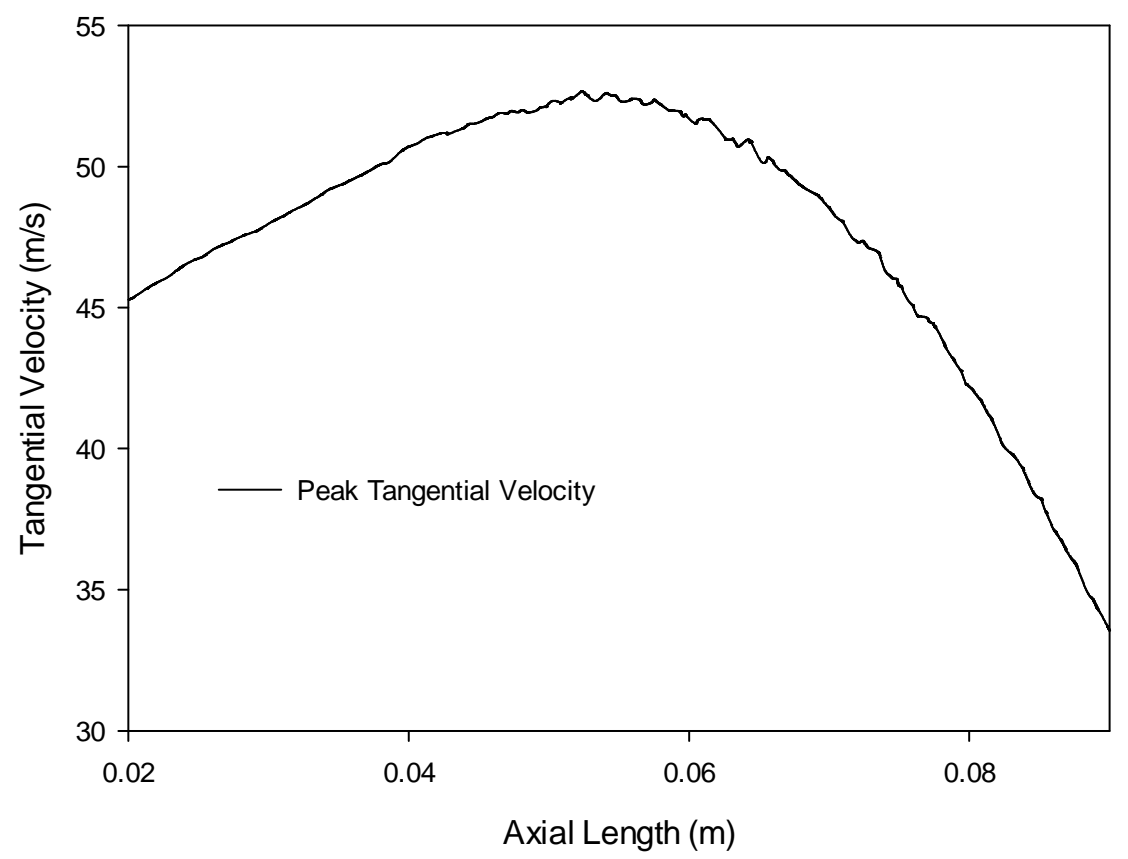

Figure 39: Case 5 - Peak Tangential Velocity

\section{Case 6: Swirl Flow With Transpiration Under an Inviscid Flow}

The last case considered in the numerical analysis mini-study is an ideal swirl flow with transpiration under an inviscid flow condition, where the main goal of this simulation is to identify the behavior of a swirl flow without the decay caused by friction and viscosity. The setup of this simulation is based on a head-end injection flow with a swirl number of 0.385 . The results displayed in Fig. 40 show the streamline profile, pressure contour profile, axial velocity contour profile, and tangential velocity contour profile. Additional detailed results of the pressure and velocity profiles are provided in Figs. 41 to 45.

Based on the results shown, radial variations of pressure and axial velocity due to the presumed centrifugal acceleration effect are present, as expected. The core flow axially and tangentially accelerates due to the radial transpiration mass that is added along the fuel grain port, while the corresponding pressure decreases. Average tangential velocity within the core flow does 
decrease moving downstream, but does not decay quite as rapidly as seen for the viscous flow cases examined earlier. Therefore, this simulation and the previous simulations for viscous flow suggest that average tangential velocity decay moving downstream may more likely be somewhat or considerably stronger in the viscous turbulent flow case. In the more realistic case, the level of decay would likely also depend in part on the magnitude of the injection separation distance $\delta$, i.e., the proximity of the principal tangential flow to the bounding fuel surface, a factor which was not examined in this CFD mini-study.

To clarify an additional simulation modelling detail, the center of the flow shown in Fig. 43 needs to reach the zero point; however, the mesh of the sample nodes used for the flow solution might not be dense enough to capture the very center of the flow. 


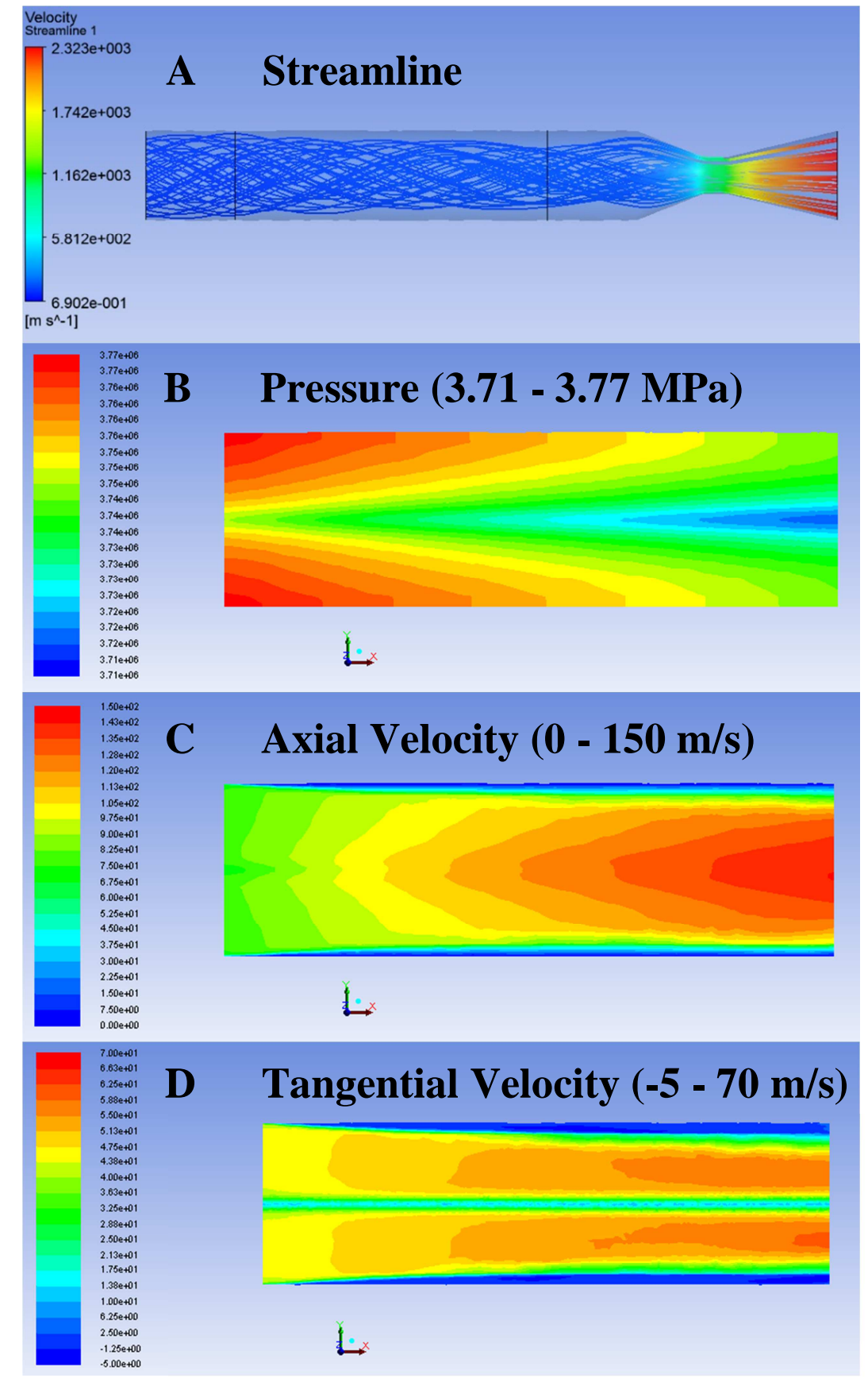

Figure 40: Case 6 - Streamlines, Pressure, Axial Velocity, and Tangential Velocity 


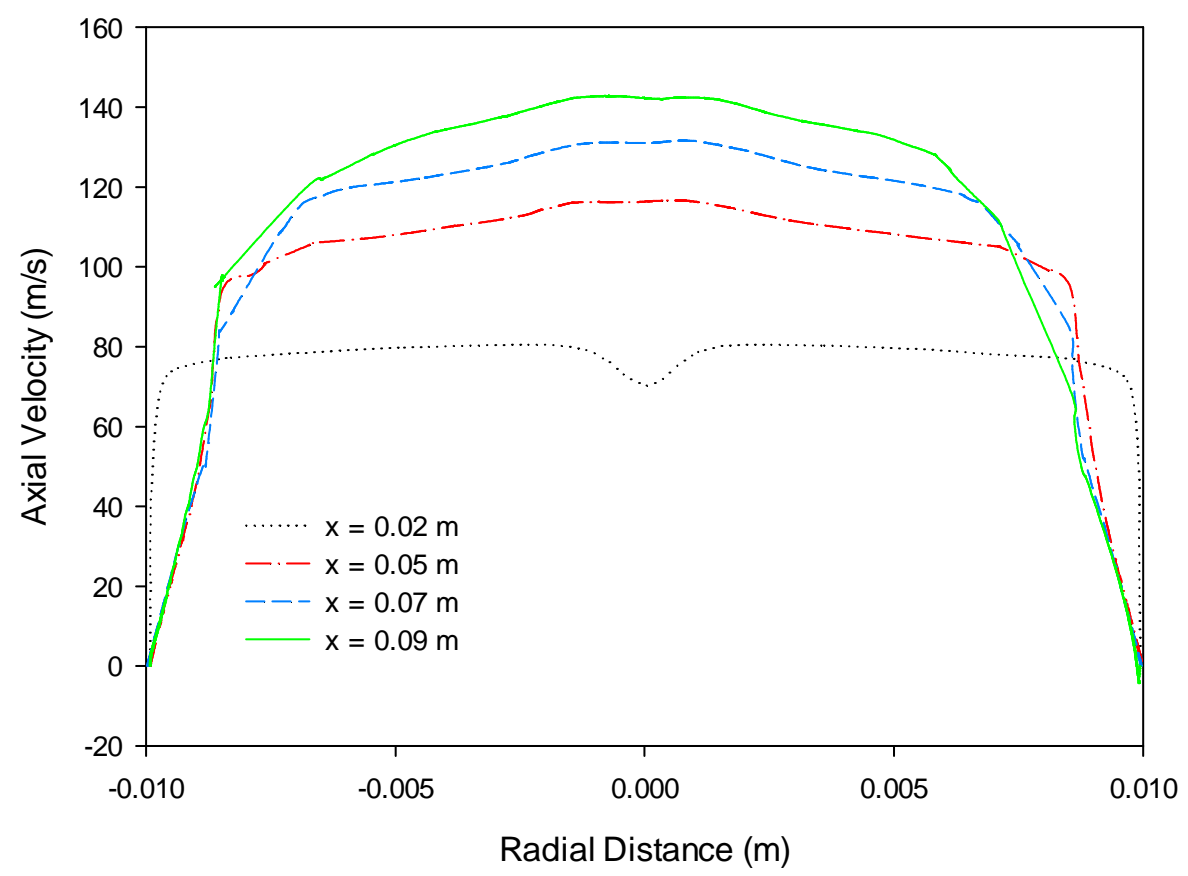

Figure 41: Case 6 - Detailed Axial Velocity Profiles

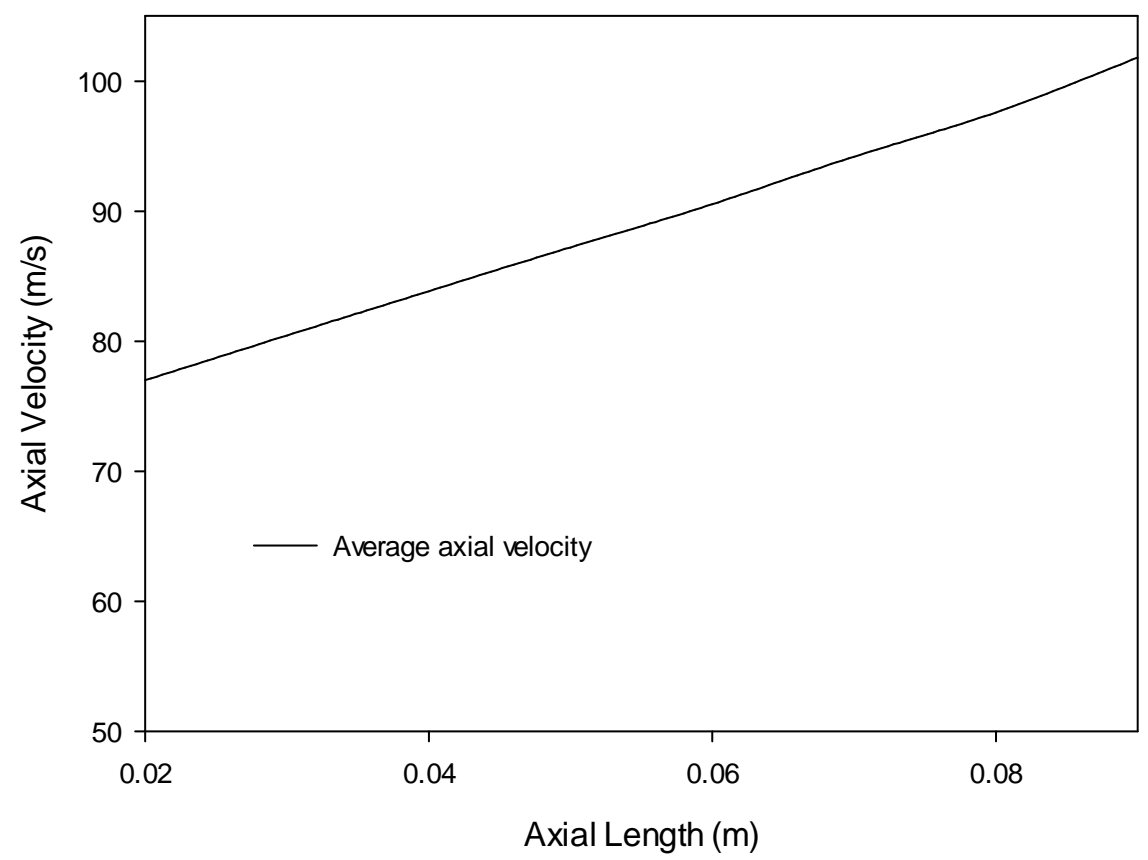

Figure 42: Case 6 - Average Axial Velocity 


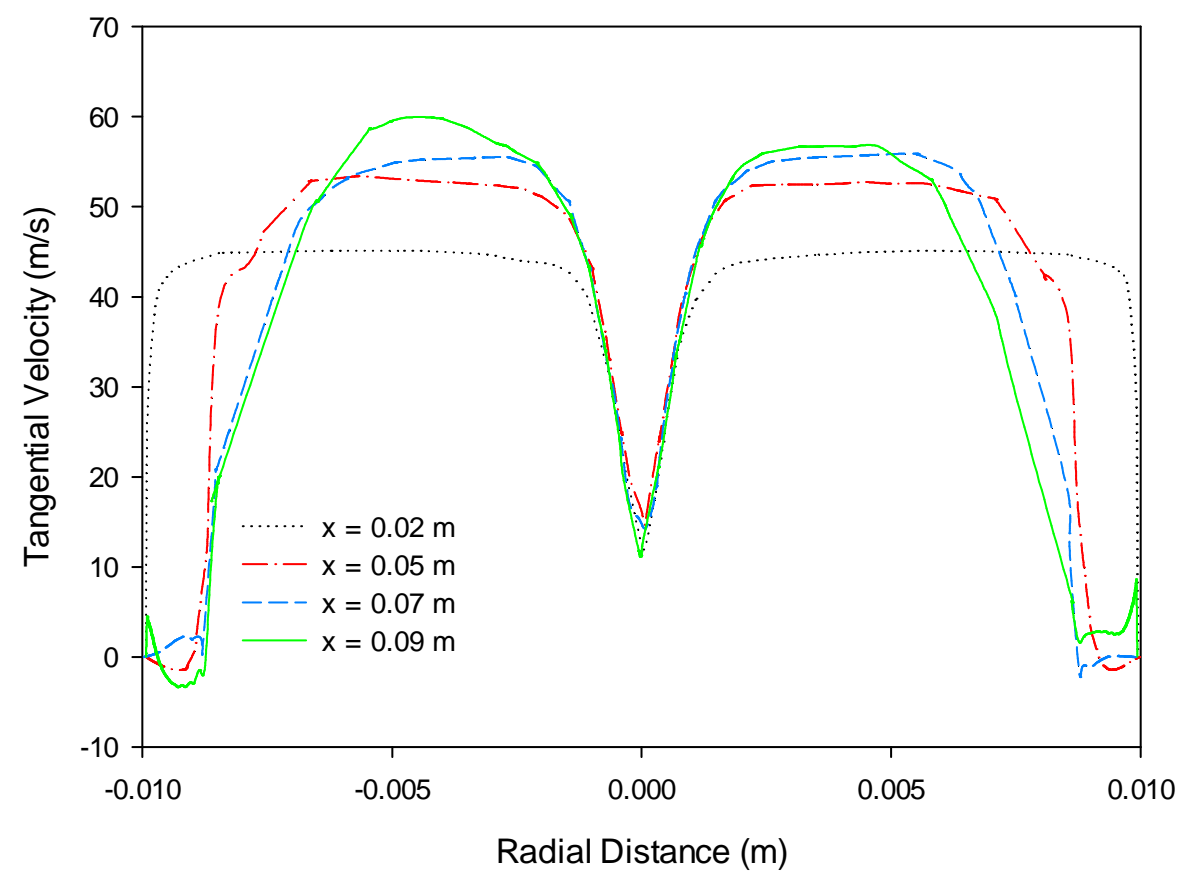

Figure 43: Case 6 - Detailed Tangential Velocity Profiles

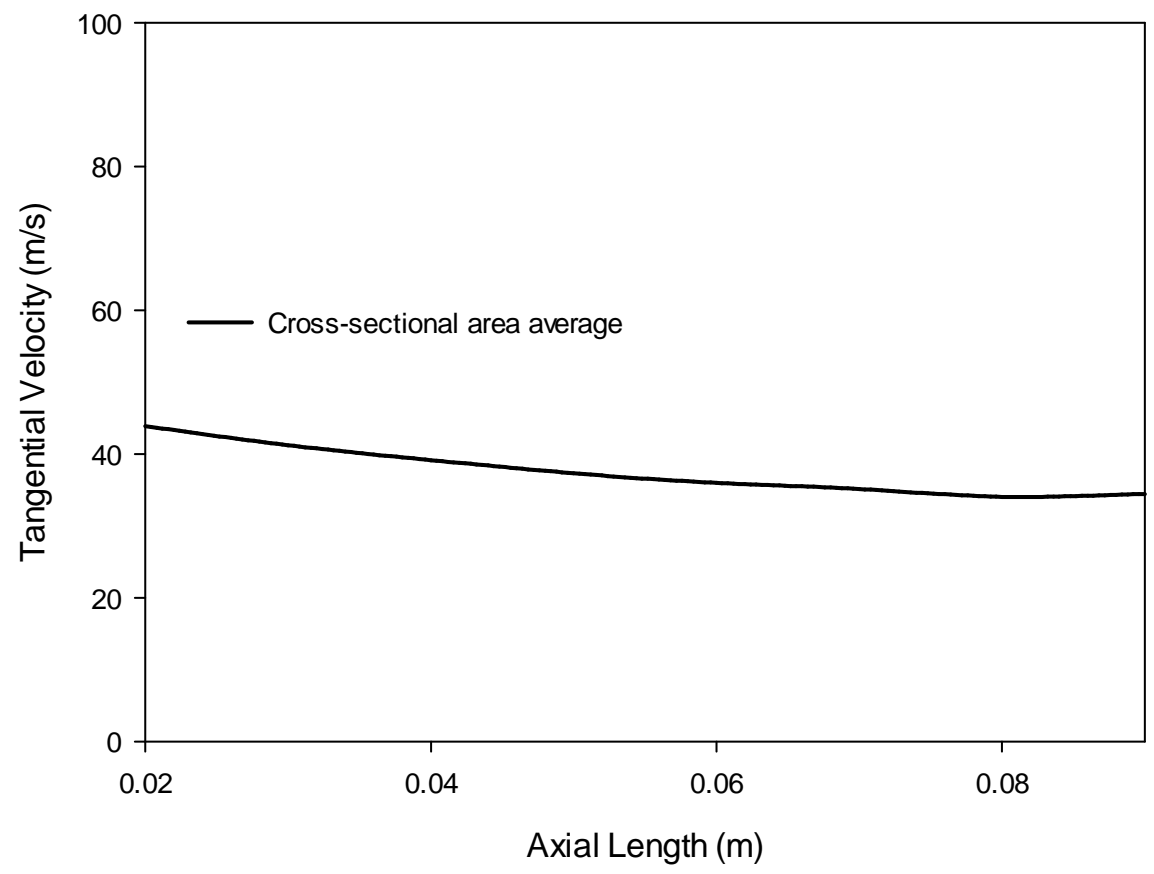

Figure 44: Case 6 - Average Tangential Velocity 


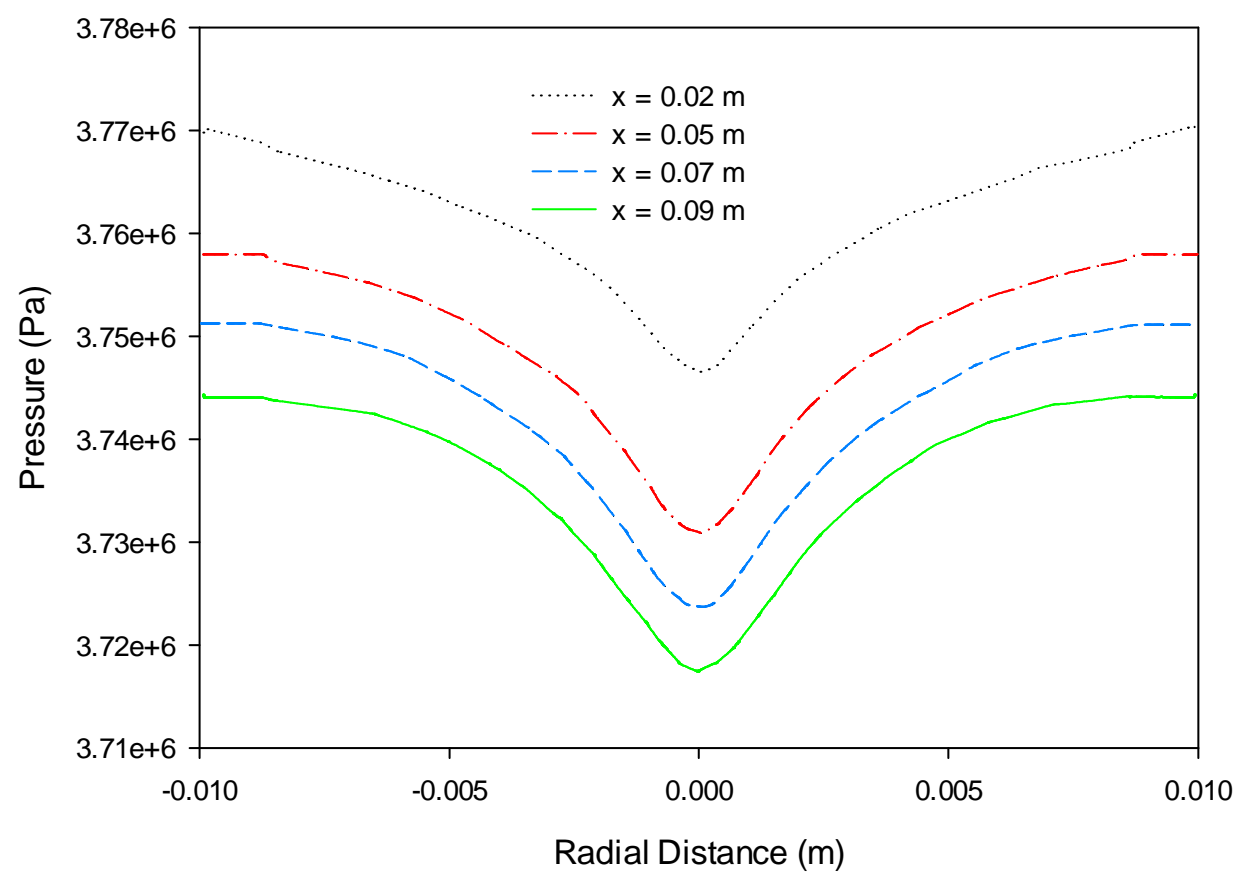

Figure 45: Case 6 - Detailed Pressure Profiles

Table 8: Case 6 - Inlet Conditions

\begin{tabular}{ll}
\hline \multicolumn{2}{c}{ Head-End Inlet Conditions } \\
\hline Mass flow rate $(\mathrm{kg} / \mathrm{s})$ & 0.09716 \\
Average Pressure $(\mathrm{Pa})$ & $3,765,611$ \\
Axial Velocity $(\mathrm{m} / \mathrm{s})$ & 77.48 \\
Tangential Velocity $(\mathrm{m} / \mathrm{s})$ & 44.74 \\
\hline
\end{tabular}




\subsection{CFD Simulation Study, Discussion and Summary}

Two characteristics associated with the operation of swirling-flow hybrid rocket engines, the decay of average tangential (swirl) velocity as one moves downstream from the engine head end, and the transpiration effect on axial and tangential velocity, have been investigated in this CFD mini-study. In this regard, one can note that three mechanisms of swirl decay have been suggested by Wilkinson et al. ${ }^{23}$ These include the expansion of gas due to the increasing temperature moving downstream in a reactive gas environment, wall friction and viscous loss, and fuel mass addition (transpiration). Since the CFD simulations done in the present mini-study were for a non-reactive ideal-gas flow, the effect of "increasing temperature" on swirl decay would not have been possible to ascertain, given the absence of the increasing temperature phenomenon itself.

The results of the simulations show that wall friction and flow viscosity can impact the flow, and possibly is a contributor to average tangential velocity decay, which in turn would potentially act to decrease the swirl intensity as one moves downstream. Other factors, like the magnitude of injection separation distance $\delta$, were not examined in the mini-study, so it would be unwise to generalize too much in regards to how much swirl decay one might encounter. One would presume that the geometry of a given experimental apparatus would have a significant influence in this regard as well.

Transpiration is observed to have at least one positive effect on the flow, to complement the other main benefits of solid-fuel combustion; the core flow experiences less friction at the bounding fuel surface. Thus, one could readily argue that Wilkinson's third "contributor" to swirl decay, transpiration, can in fact be a mitigating factor, acting to reduce the influence of wall friction, and possibly flow viscosity as well. This comment is referring to the correction for the transpiration effect in the model derivation. Also, the observed increasing and decreasing profile of the tangential velocity as a result of transpiration helps explain the possible sources of discrepancies due to the length of the fuel grain, e.g., a short fuel grain possibly contains a faster tangential flow, while a long fuel grain contains a slower tangential flow by average. 


\section{Influence of Input Parameters}

To better understand the results produced by the fuel regression rate model ${ }^{11}$, and to check the influence of relevant parameters on the resulting estimate of regression rate, variations on the value of input parameters are investigated in this section. The results are illustrated in terms of fuel regression rate versus the total axial mass flux, where the variations of the results due to changing values of the selected parameters by $+/-10 \%$ (as a preliminary starting point used in the analysis) are also plotted in comparison, as shown in Figs. 46 to 56. The sample rocket engine's geometry and the propellant properties are set based on an engine with similar testing conditions as that used in Chapter 6, where the list of input parameters, their initial values, as well as the variation results, are shown in Table 9. Additionally, more cases for the input parameter study may be carried out using the computer program provided in Appendix B. The program allows for a quick generation of the model results, allowing one to observe the change of the regression rate results due to combined parameters, or for comparing different types of propellant, especially if one needs to investigate the effect of changing swirl numbers.

In changing their respective values, many of the input parameters are shown to produce proportionally comparable changes in the fuel regression rate value, regardless of swirl number value, except for those input parameters used in the Colebrook friction factor equations, including $\mu, \varepsilon, d_{x}$, and $d_{\theta}$. The relatively constant percentage change implies that the change in regression rate becomes more significant in absolute terms as the swirl number becomes larger. It is clear that some input parameters have more of an influence than the others. From the result, $T_{f} T_{s}, k, \rho_{s}$, and $\mu$, which are the relevant propellant properties in both the solid and gas phase, seem to vary the results of regression rate at a much greater rate than geometrical factors such as the hydraulic diameters and fuel surface roughness. Given this background, the average or estimated values that represent the propellant properties should be carefully selected. Furthermore, the aforementioned parameters signify the importance of the heat transfer processes and the contribution of the flow viscosity (through Reynolds analogy) to the characteristics of regression rate. 
As a final note to conclude this section, the trends of the parameters being investigated in the present study shows conformity with the trends identified for the regression rate model proposed by another institution. ${ }^{24}$ Examples of the effects investigated in their study include the scale effect, which reduces regression rate as the chamber becomes larger, and the increasing initial propellant temperature, which gives a positive effect to the regression rate.

Table 9: Percentage Increase of Regression Rate by Each Parameter (at G = 500 kg/s-m ${ }^{2}$ )

\begin{tabular}{|c|c|c|c|c|c|c|c|c|}
\hline \multicolumn{3}{|c|}{ Swirl Number } & \multicolumn{2}{|c|}{$S=0$} & \multicolumn{2}{|c|}{$S=0.385$} & \multicolumn{2}{|c|}{$S=0.667$} \\
\hline Variation & Initial Value & & $-10 \%$ & $+10 \%$ & $-10 \%$ & $+10 \%$ & $-10 \%$ & $+10 \%$ \\
\hline$C_{p} \quad(\mathrm{~J} / \mathrm{kg}-\mathrm{K})$ & 1695 & $\downarrow$ & $4.53 \%$ & $-4.08 \%$ & $4.53 \%$ & $-4.08 \%$ & $4.53 \%$ & $-4.08 \%$ \\
\hline$C_{s} \quad(\mathrm{~J} / \mathrm{kg}-\mathrm{k})$ & 2100 & $\downarrow$ & $6.11 \%$ & $-5.37 \%$ & $6.11 \%$ & $-5.37 \%$ & $6.11 \%$ & $-5.37 \%$ \\
\hline$T_{f} \quad(\mathrm{~K})$ & 3000 & $\uparrow$ & $-8.83 \%$ & $7.95 \%$ & $-8.83 \%$ & $7.95 \%$ & $-8.83 \%$ & $7.95 \%$ \\
\hline$T_{s} \quad(\mathrm{~K})$ & 950 & $\downarrow$ & $11.69 \%$ & $-10.05 \%$ & $11.69 \%$ & $-10.05 \%$ & $11.69 \%$ & $-10.05 \%$ \\
\hline$T_{i} \quad(\mathrm{~K})$ & 284 & $\uparrow$ & $-2.37 \%$ & $2.5 \%$ & $-2.37 \%$ & $2.5 \%$ & $-2.37 \%$ & $2.5 \%$ \\
\hline$k \quad(\mathrm{~W} / \mathrm{m}-\mathrm{K})$ & 0.2 & $\uparrow$ & $-10 \%$ & $10 \%$ & $-10 \%$ & $10 \%$ & $-10 \%$ & $10 \%$ \\
\hline$\rho_{s} \quad\left(\mathrm{~kg} / \mathrm{m}^{3}\right)$ & 920 & $\downarrow$ & $11.11 \%$ & $-9.09 \%$ & $11.11 \%$ & $-9.09 \%$ & $11.11 \%$ & $-9.09 \%$ \\
\hline$\mu\left(\mathrm{N} \mathrm{s} / \mathrm{m}^{2}\right)$ & $8 \times 10-5$ & $\downarrow$ & $9.45 \%$ & $-7.80 \%$ & $9.32 \%$ & $-7.70 \%$ & $9.44 \%$ & $-7.79 \%$ \\
\hline$\varepsilon \quad(\mu \mathrm{m})$ & 5 & $\uparrow$ & $-0.64 \%$ & $0.63 \%$ & $-0.57 \%$ & $0.56 \%$ & $-0.65 \%$ & $0.64 \%$ \\
\hline$d_{x}(\mathrm{~cm})$ & 3.175 & $\downarrow$ & $2.24 \%$ & $-1.96 \%$ & $1.35 \%$ & $-1.18 \%$ & $1.09 \%$ & $-0.96 \%$ \\
\hline$d_{\theta}(\mathrm{cm})$ & 2.54 & $\uparrow$ & 0 & 0 & $0.94 \%$ & $-0.82 \%$ & $1.17 \%$ & $-1.03 \%$ \\
\hline
\end{tabular}




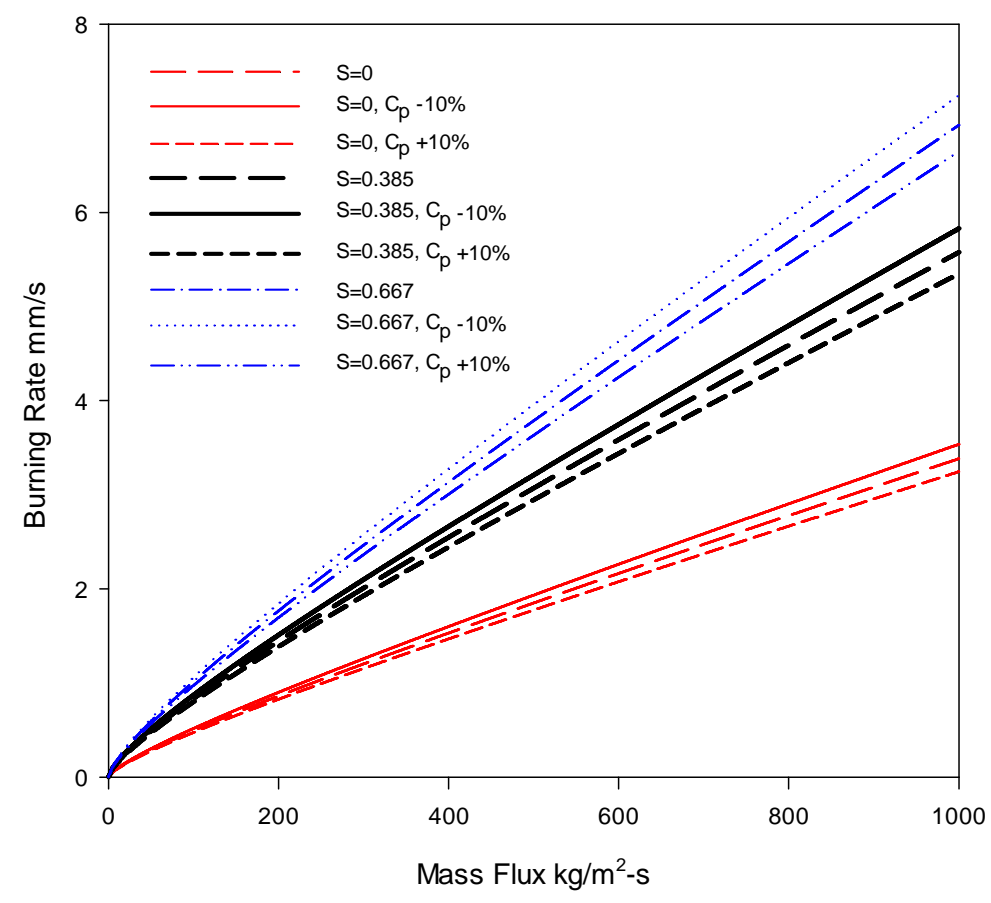

Figure 46: Effect of Variation of $C_{p}$

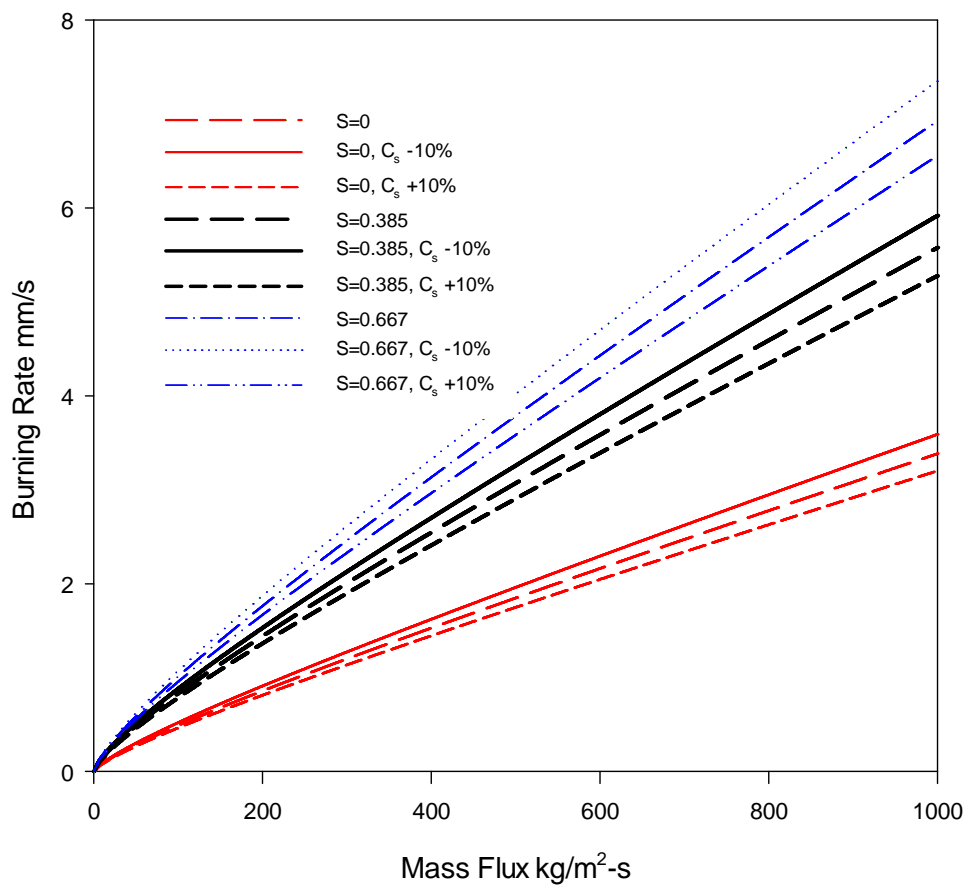

Figure 47: Effect of Variation of $C_{s}$ 


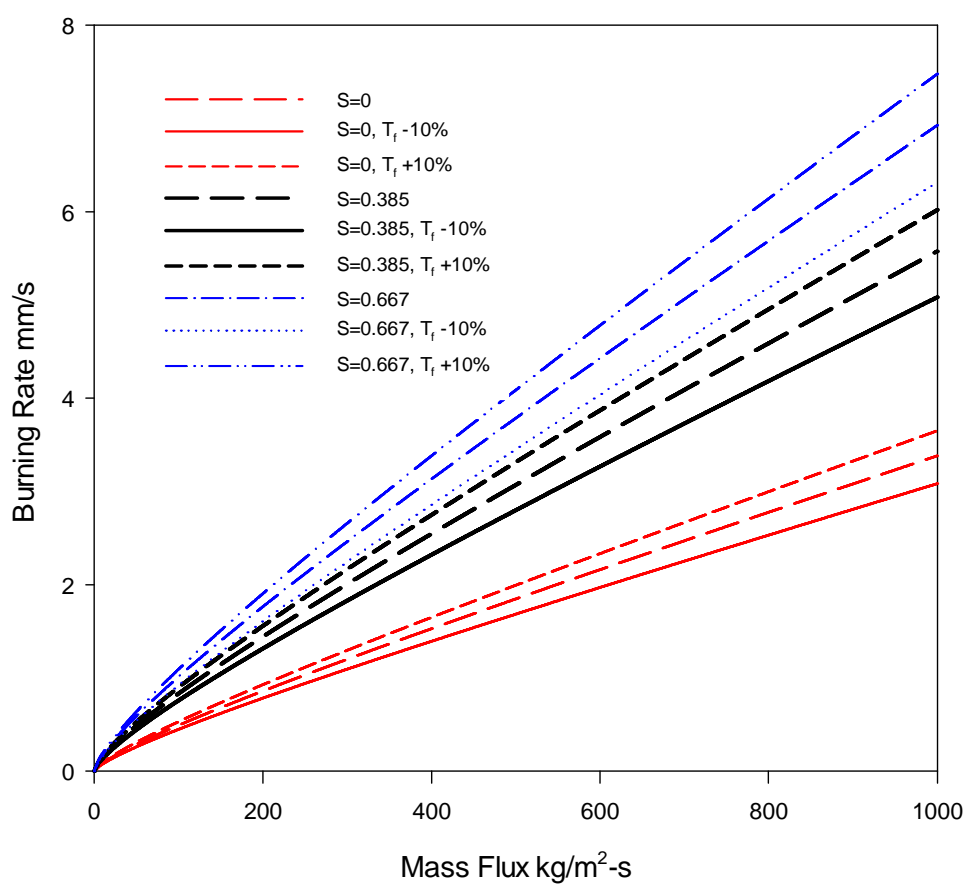

Figure 48: Effect of Variation of $T_{f}$

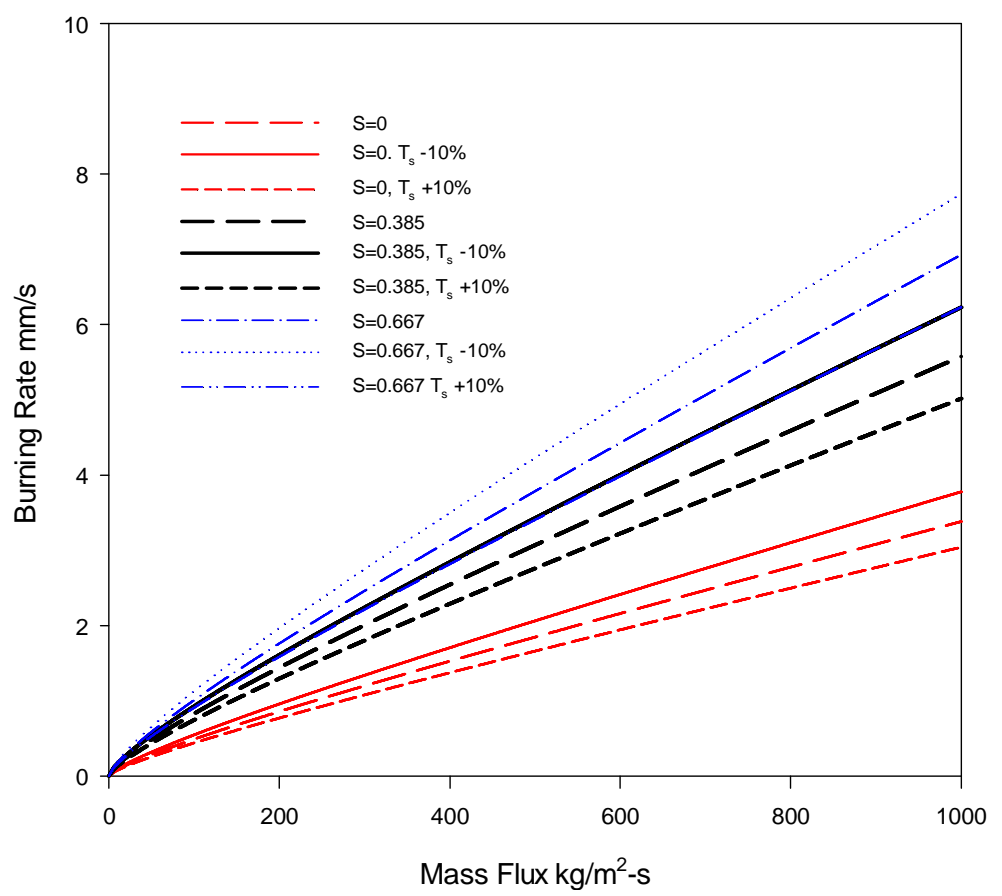

Figure 49: Effect of Variation of $T_{s}$ 


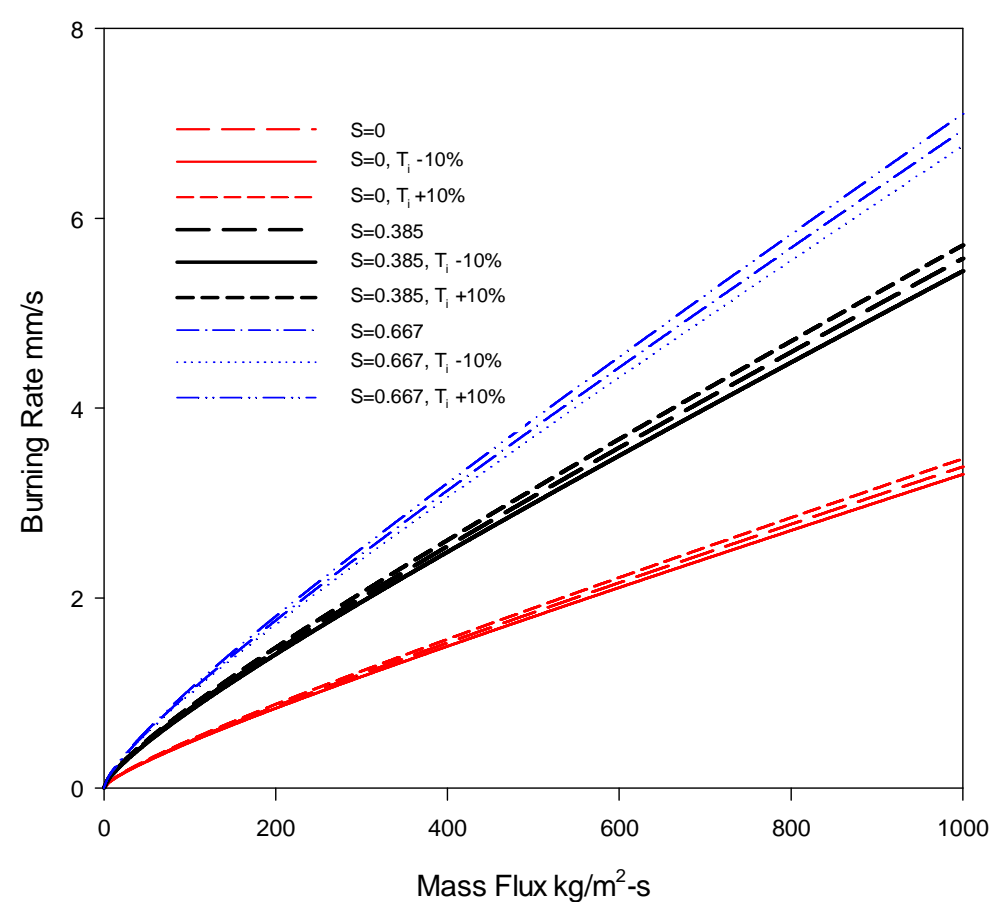

Figure 50: Effect of Variation of $T_{i}$

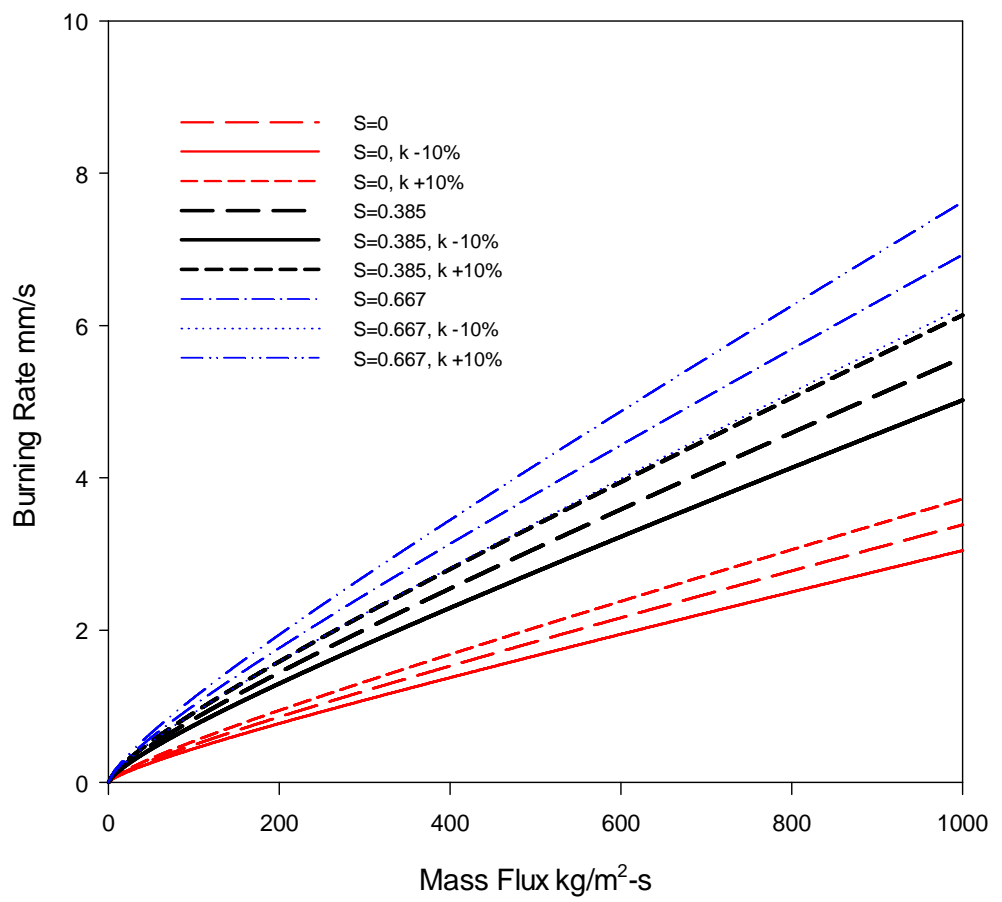

Figure 51: Effect of Variation of $k$ 


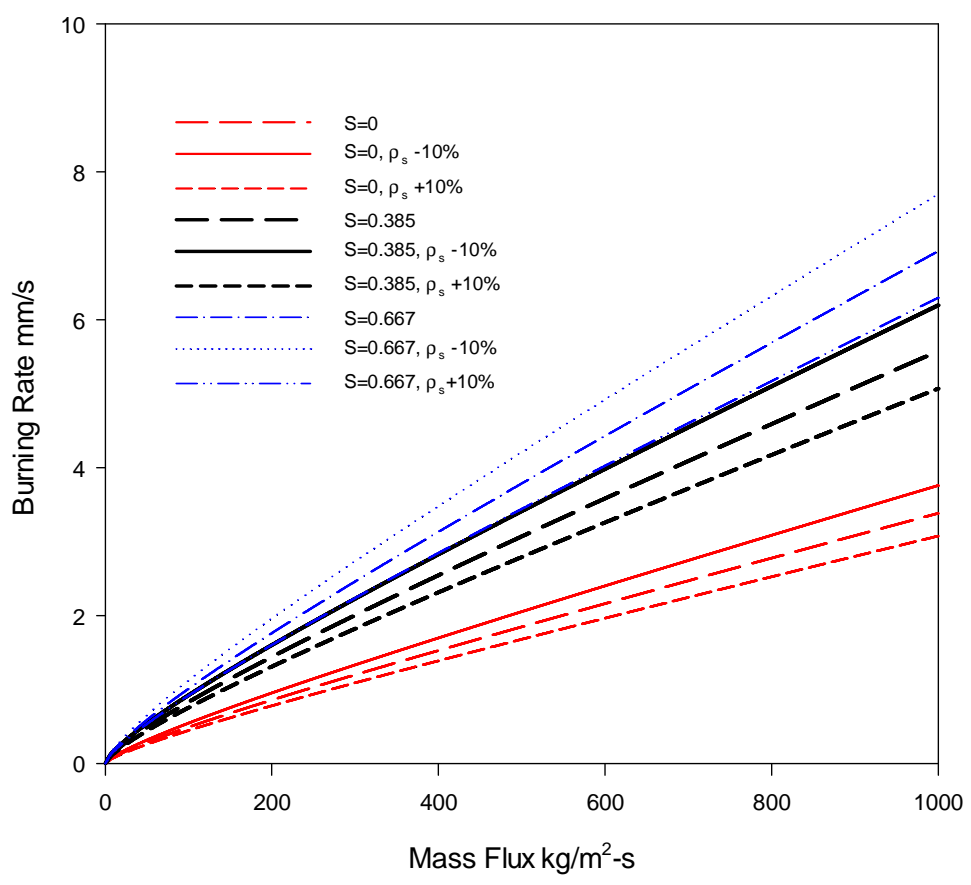

Figure 52: Effect of Variation of $\rho_{s}$

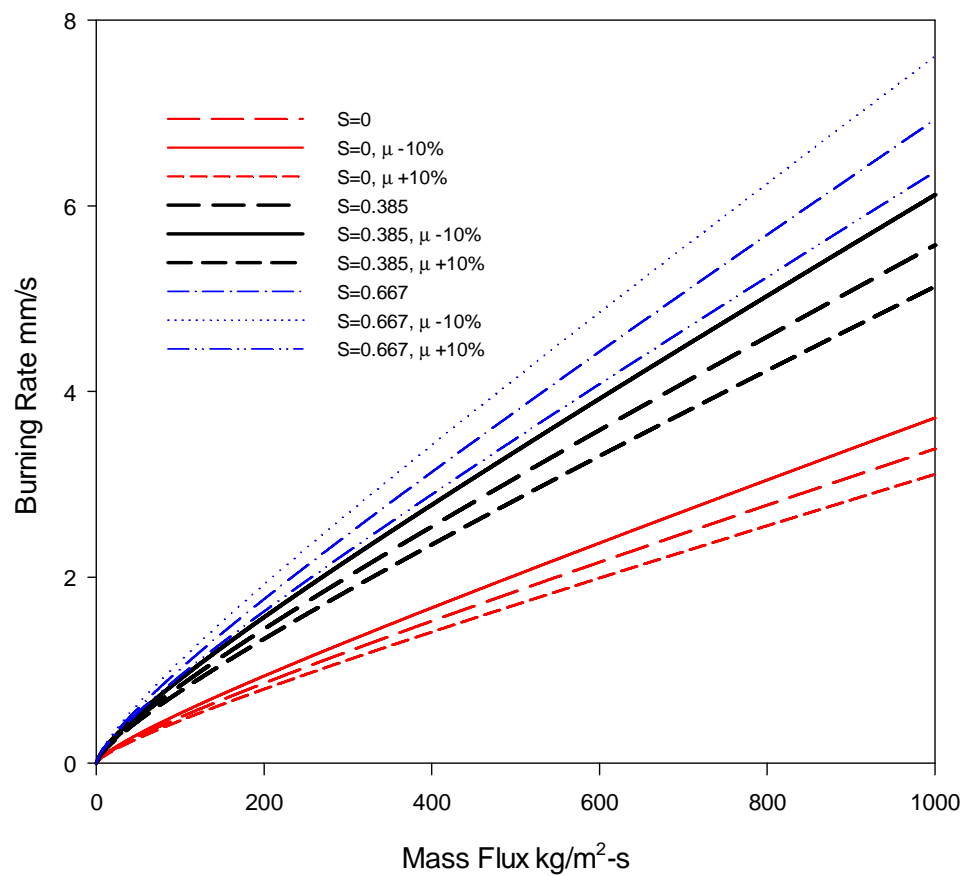

Figure 53: Effect of Variation of $\mu$ 


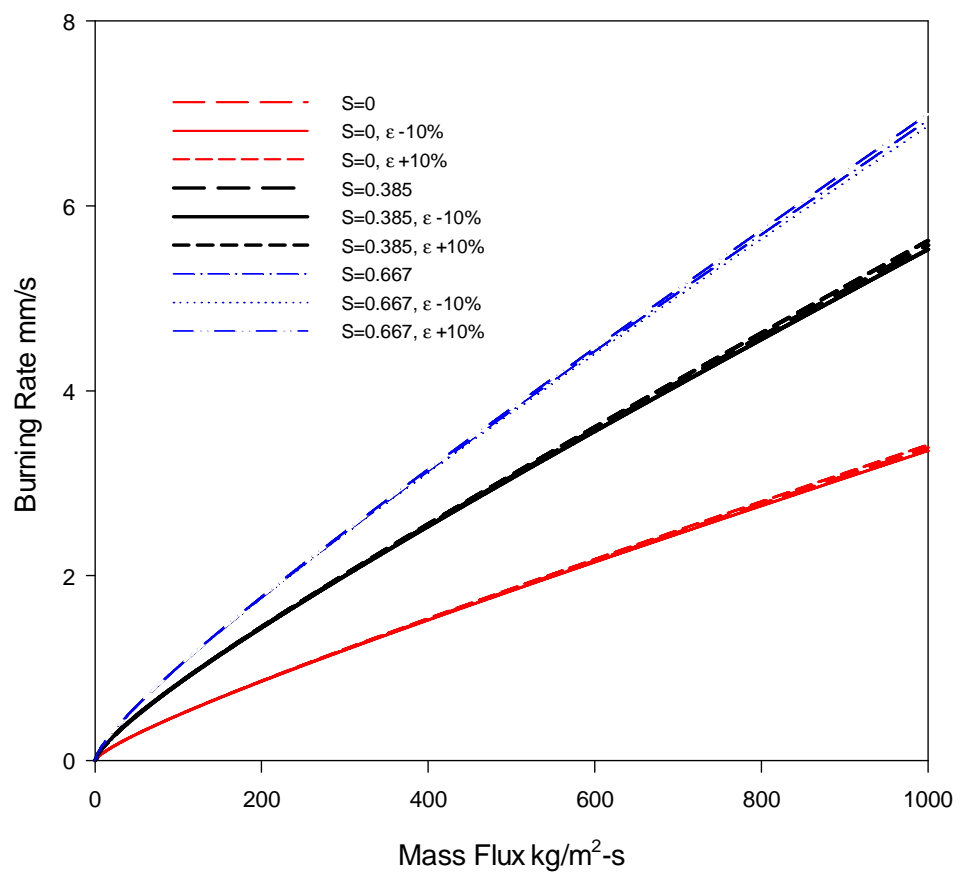

Figure 54: Effect of Variation of $\varepsilon$

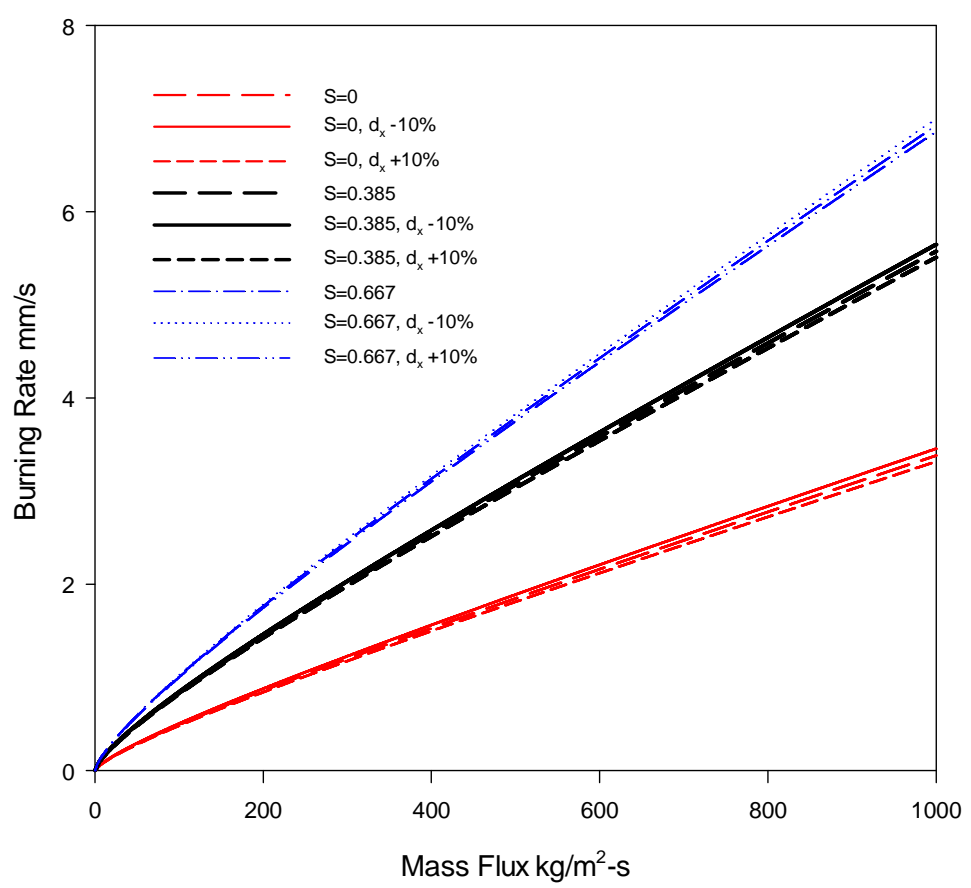

Figure 55: Effect of Variation of $d_{x}$ 


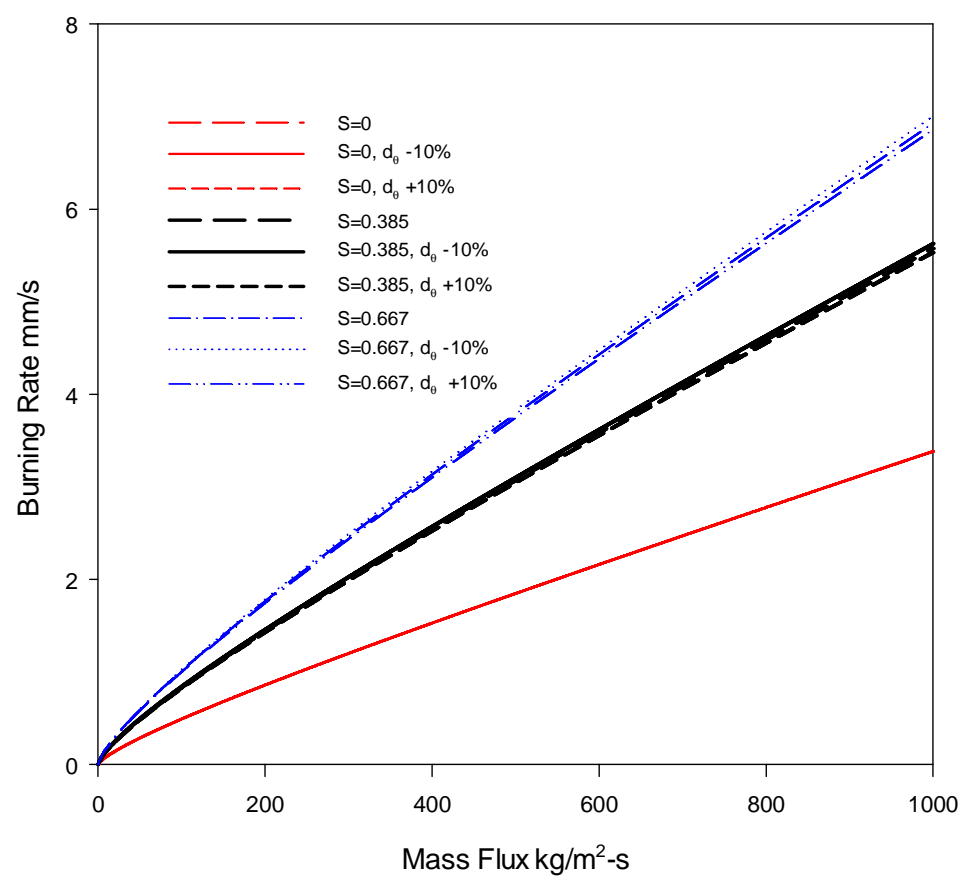

Figure 56: Effect of Variation of $d_{\theta}$ 


\section{Conclusion and Recommendations}

A functional fuel regression rate estimation model for a swirling-flow hybrid rocket engine has been developed using the convective heat feedback approach. The effective convective heat transfer coefficients, based on the axial and tangential (swirl) flow components, play a key role in the calculation procedure. The influence of effective hydraulic diameters, transpiration, and fuel surface roughness are included in the analysis.

Experimental firing data for eight engine configurations were compared with the results generated by the predictive model for regression rate. The data involved three combinations of propellant: paraffin/GOx, HDPE/N2O, and HTPB/GOx, and engines of differing sizes. The predicted results comply with the qualitative and quantitative trends of the experimental data, for the most part. With respect to discrepancies, sometimes it is, at least in part, a factor of the given experimental apparatus used, or the measurement technique used by the experimenters.

For gaining additional understanding of swirl flows in hybrid rocket engines, a CFD simulation model was used to assess the relevant characteristics of the engine flow, including the decay of swirl intensity, and the effect of transpiration on axial and tangential velocity as one moves downstream. For example, the result of a swirl flow simulation run with transpiration showed an overall decay of average tangential velocity in moving from the head end to the nozzle end of the engine.

In the last part of the study, the sensitivity of the estimate of fuel regression rate with respect to values used for several relevant input parameters used in the model was tested. The solid and gas properties associated with the propellant are shown to have more of an influence on the predicted value of fuel regression rate than the parameters used for defining the grain geometry. 
Future research for swirling-flow hybrid rocket engines at Ryerson University may include the conducting of test firings to further validate or improve upon the preliminary work outlined in this thesis. Design parameters that were not looked at in too much detail in this thesis, such as the length of the fuel grain, may be worth further investigation, as relates to influencing swirl behavior. Lastly, a more elaborate CFD study may be warranted, with a more definitive representation of the swirl injection input, to better understand the actual flow field inside of a hybrid rocket engine that is utilizing swirl to augment the fuel regression rate. 


\section{Appendix A: Sample Results from Quasi-Steady Analysis}

In a parallel RU study entitled "Alternative Thrust Modulation Techniques for Solid and Hybrid Rockets," 19 the internal ballistic performance of a proposed hybrid rocket engine, which uses swirling-flow head-end injection and a cylindrical fuel grain, was evaluated in comparison with the baseline axial flow injection method. Sample performance results of the engine predicted by the quasi-steady internal ballistic approach used in the RU computer program QSHYB are provided in Figs. 57 to 59, including the head-end pressure, thrust and stoichiometric length computed with respect to time. The type of propellant used for the engine is $\mathrm{N}_{2} \mathrm{O} / \mathrm{HTPB}$. The fuel grain is located within the propulsive section of the rocket (this section is approximately $1.55 \mathrm{~m}$ in length). The mid-firing diameter of the fuel port used in the analysis is $8 \mathrm{~cm}$, while the corresponding tangential hydraulic diameter at mid-firing is $10 \mathrm{~cm}$. In the case of the swirl flow being applied, the swirl intensity being investigated involves an oxidizer injection with a swirl number of 2 , which is activated during the period of $3 \mathrm{~s}<t<10 \mathrm{~s}$ of the firing time. The oxidizer mass flow rate of the baseline flow is $0.385 \mathrm{~kg} / \mathrm{s}$, while the oxidizer mass flow rate is further augmented to $0.485 \mathrm{~kg} / \mathrm{s}$ in the swirl flow case.

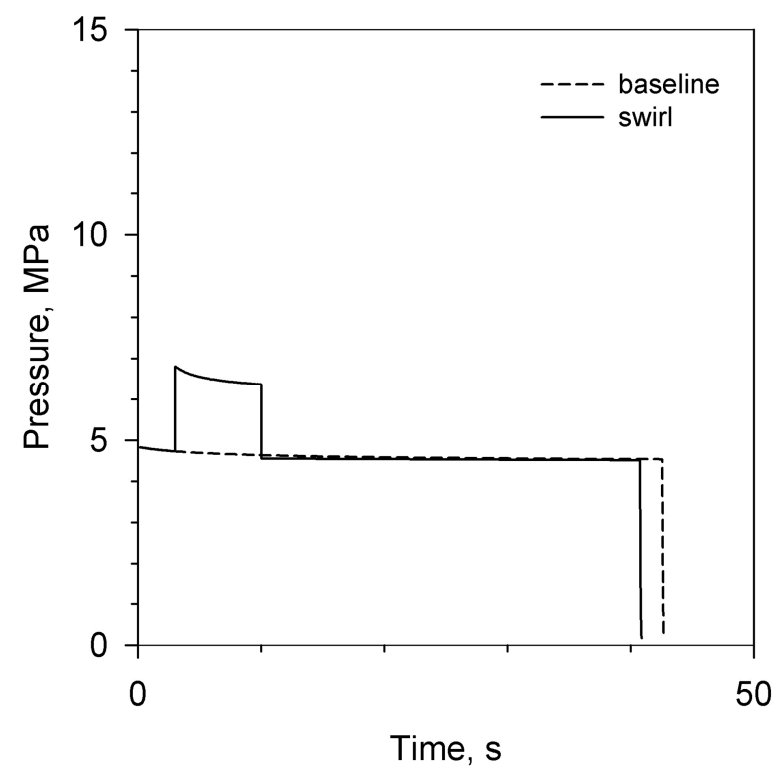

Figure 57: Head-End Pressure Prediction by QSHYB of a Swirl Flow $(S=2$, at $3 \mathrm{~s}<t<10 \mathrm{~s})$. Based on $\mathrm{N}_{2} \mathrm{O} / \mathrm{HTPB}$ Propellant ${ }^{19}$ 


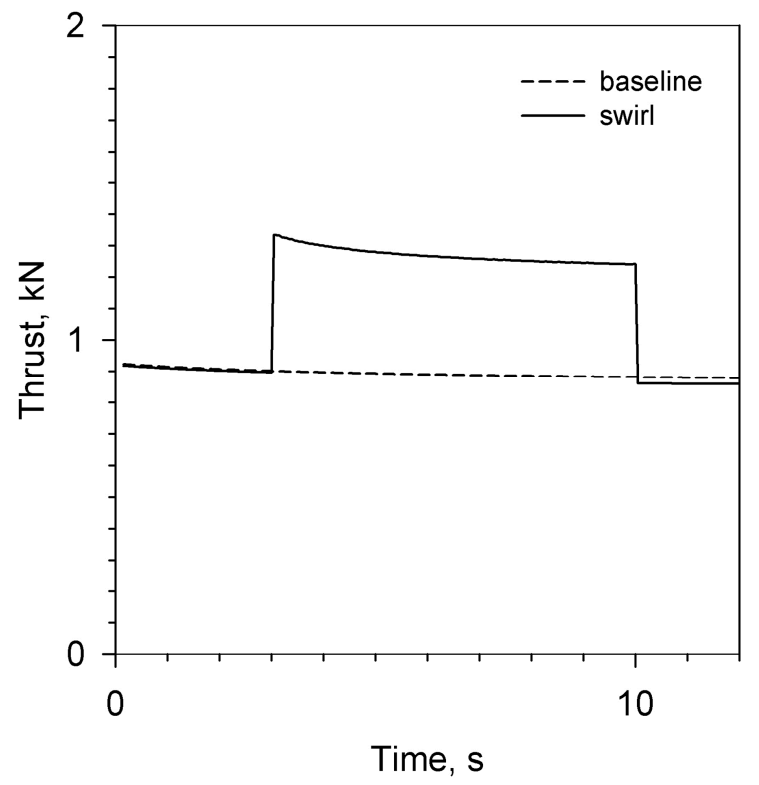

Figure 58: Thrust Prediction by QSHYB of a Swirl Flow ( $S=2$, at $3 \mathrm{~s}<t<10 \mathrm{~s})$. Based on $\mathrm{N}_{2} \mathrm{O} / \mathrm{HTPB}$ Propellant ${ }^{19}$

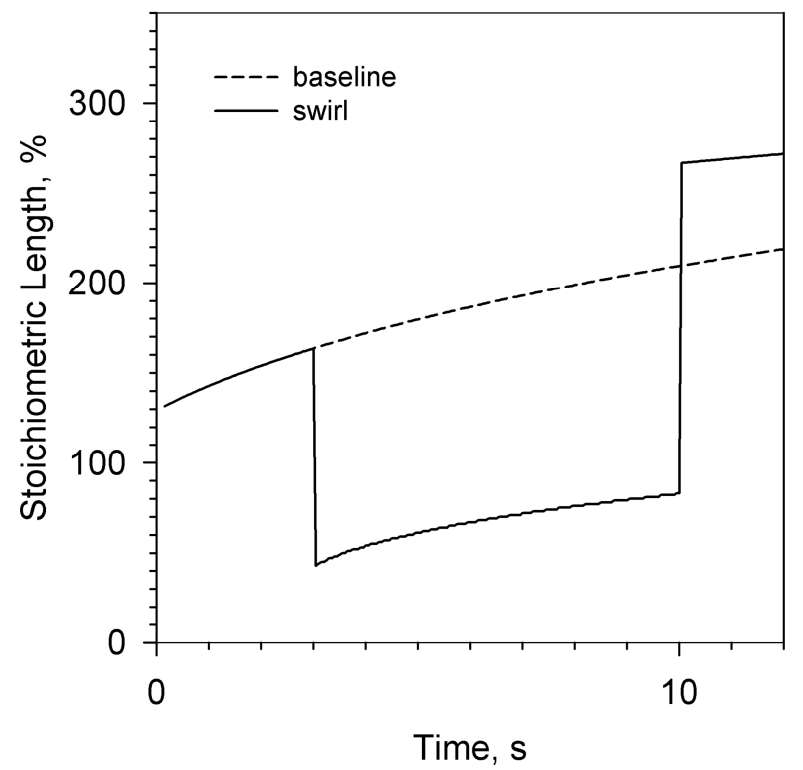

Figure 59: Stoichiometric Length Prediction by QSHYB of a Swirl Flow ( $S=2$, at $3 \mathrm{~s}<t<10 \mathrm{~s}$ ). Based on $\mathrm{N}_{2} \mathrm{O} / \mathrm{HTPB}$ Propellant ${ }^{19}$ 


\section{Appendix B: Mini-Program for Input Parameter Study}

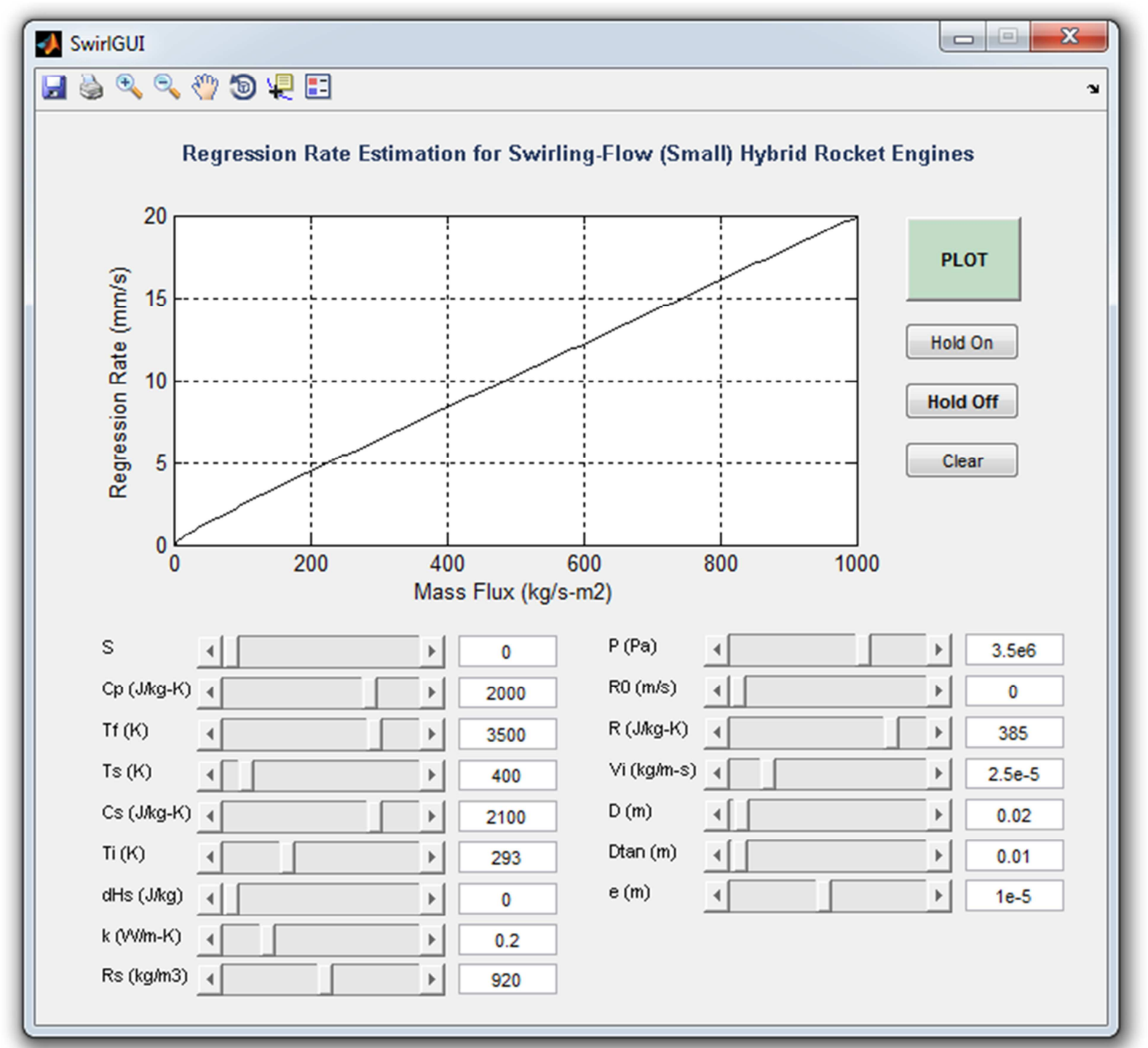

Figure 60: User Interface for Input-Parameter Study Program 


\section{MATLAB Code (Main Calculation Algorithm):}

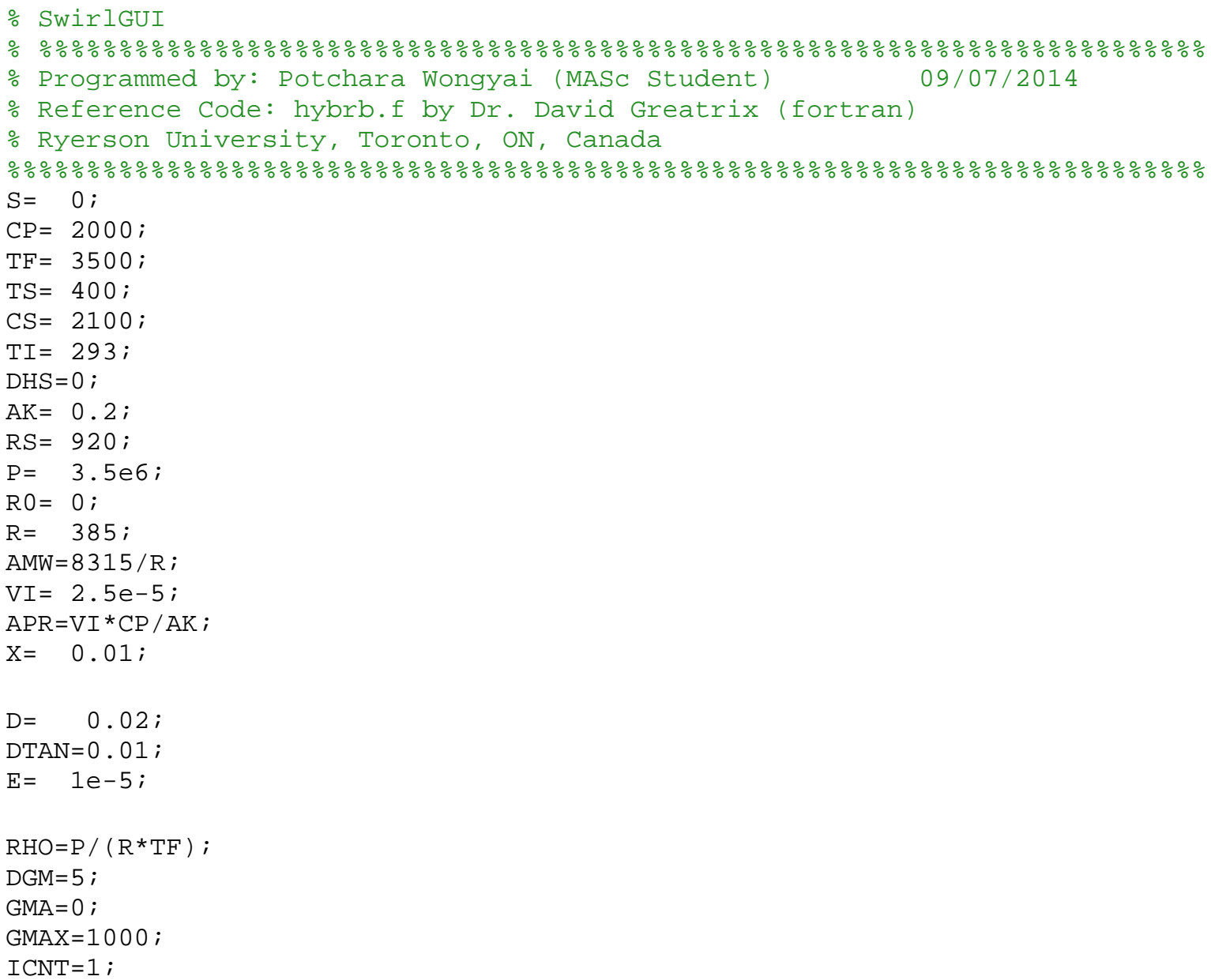




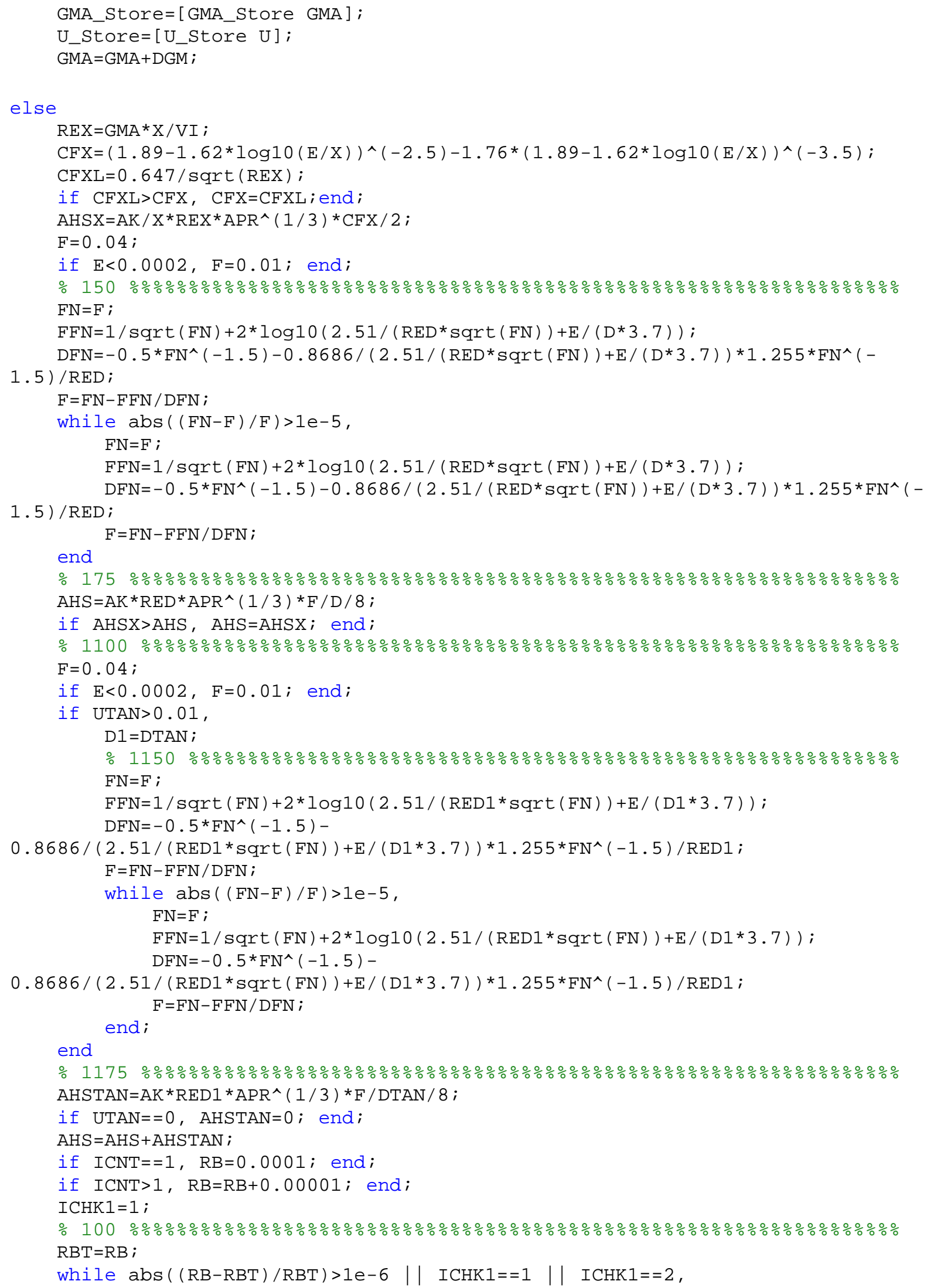




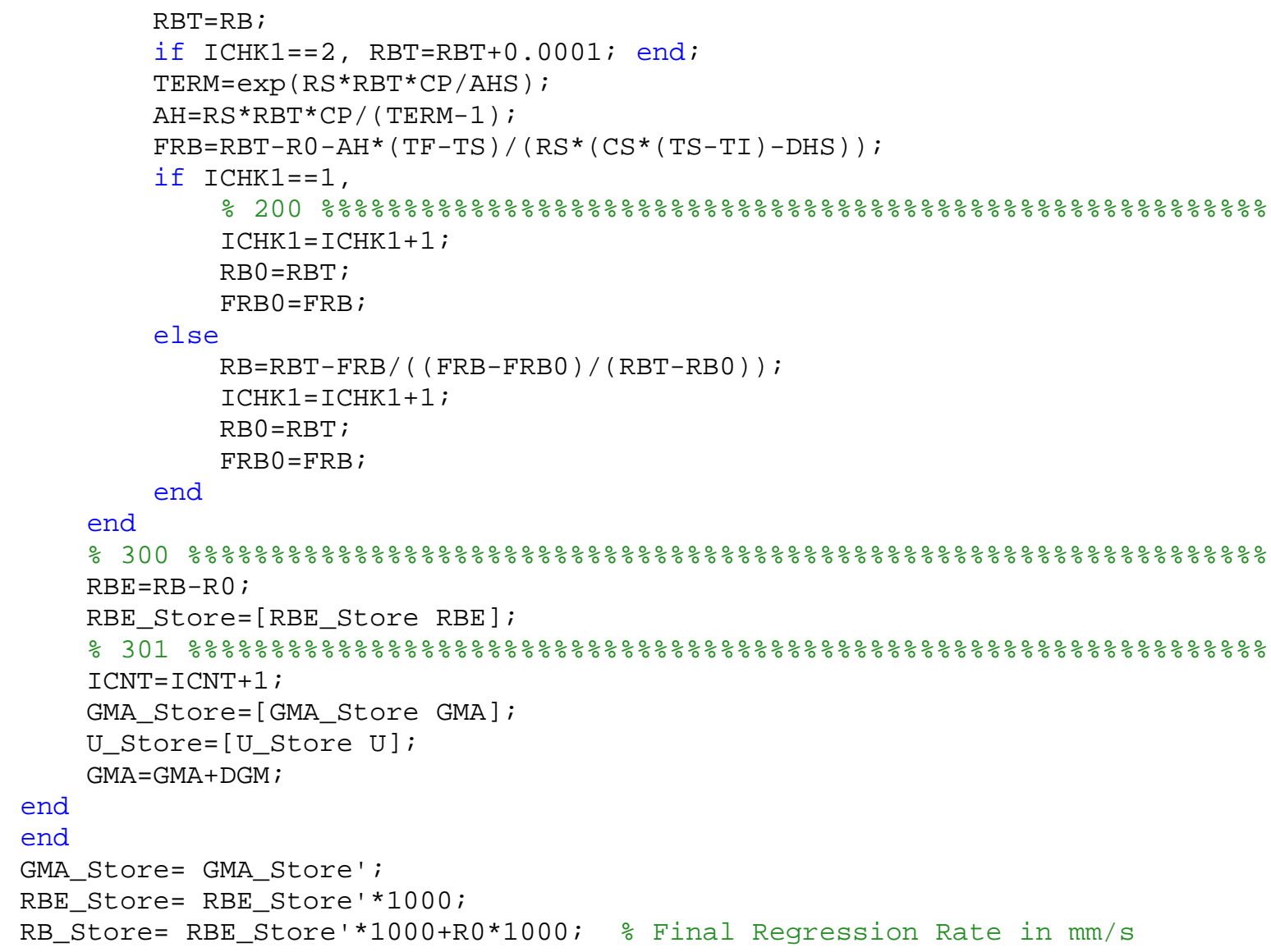




\section{References}

1. Chiaverini, M.J., "Review of Solid-Fuel Regression Rate Behavior in Classical and Nonclassical Hybrid Rocket Motors," Ch. 2 in Fundamentals of Hybrid Rocket Combustion and Propulsion, edited by Chiaverini, M.J. and Kuo, K.K., Vol. 218, Progress in Astronautics \& Aeronautics series, AIAA Publications, Reston, VA, 2007, pp. 37-125.

2. Knuth, W.H., Gramer, D.J., Chiaverini, M.J. and Sauer, J.A., "Development and Testing of a Vortex-Driven, High-Regression Rate Hybrid Rocket Engine," $34^{\text {th }}$ AIAA/ASME/SAE/ ASEE Joint Propulsion Conference, AIAA Paper No. 98-3507, Cleveland, July 13-15, 1998.

3. Yuasa, S., Shimada, O., Imamura, T., Tamura, T. and Yamamoto, K., "A Technique for Improving the Performance of Hybrid Rocket Engines," $35^{\text {th }}$ AIAA/ASME/SAE/ ASEE Joint Propulsion Conference, AIAA Paper No. 99-2322, Los Angeles, June 20-24, 1999.

4. Hikone, S., Maruyama, S., Isiguro, T. and Nakagawa, I., "Regression Rate Characteristics and Burning Mechanism of Some Hybrid Rocket Fuels," $46^{\text {th }}$ AIAA/ASME/SAE/ASEE Joint Propulsion Conference, AIAA Paper No. 2010-7030, Nashville, July 25-28, 2010.

5. Pucci, J.M., "The Effects of Swirl Injector Design on Hybrid Flame-Holding Combustion Instability," $38^{\text {th }}$ AIAA/ASME/SAE/ASEE Joint Propulsion Conference, AIAA Paper No. 20023578, Indianapolis, July 7-10, 2002.

6. Lee, T.S. and Potapkin, A., "The Performance of a Hybrid Rocket with Swirling GOx Injection," Proceedings of the $11^{\text {th }}$ International Conference on Methods of Aerophysical Research, Novosibirsk (Russia), July 1-7, 2002, pp. 126-132.

7. Greatrix, D.R., Powered Flight, Springer-Verlag London Limited, 2012, pp. 293-434.

8. Chiaverini, M.J., Serin, N., Johnson, D., Lu, Y.C., Kuo, K.K. and Risha, G.A., "Regression Rate Behavior of Hybrid Rocket Solid Fuels," Journal of Propulsion \& Power, Vol. 16, No. 1, Jan.-Feb. 2000, pp. 125-132.

9. Ziliac, G. and Karabeyoglu, M.A., "Hybrid Rocket Fuel Regression Data and Modeling," $42^{\text {nd }}$ AIAA/ASME/SAE/ ASEE Joint Propulsion Conference, AIAA Paper No. 2006-4504, Sacramento, July 9-12, 2006.

10. Greatrix, D.R., "Regression Rate Estimation for Standard-Flow Hybrid Rocket Engines," Aerospace Science \& Technology, Vol. 13, No. 7, Oct./Nov. 2009, pp. 358-363.

11. Wongyai, P. and Greatrix, D.R.,"Regression Rate Estimation for Swirling-Flow Hybrid Rocket Engine," 50th AIAA Joint Propulsion Conference, AIAA Paper No. 2014-3750, Cleveland, July 27th - 30th, 2014. 
12. Greatrix, D.R. and Gottlieb, J.J., "Erosive Burning Model for Composite-Propellant Rocket Motors with Large Length-to-Diameter Ratios," Canadian Aeronautics and Space Journal 33 (3) (Sept.1987) 133-142.

13. Mickley H.S., Ross R.C., Squyer A.L. and Stewart W.F., "Heat, mass, and momentum transfer for flow over a flat plate with blowing or suction," NACA TN3208, July 1954.

14. Greitzer, E.M., Tan, C.S. and Graf, M.B, Internal Flow-Concepts and Applications, Cambridge University Press, Cambridge (U.K.), 2004, p. 438.

15. Gupta, A. K., Lilley, D. G., and Syred, N., Swirl Flows, Abacus Press, 1984, pp. 16-18.

16. Daisuke, S., Yuasa, S., Hirata, K., Sakurai, T. and Shiraishi, N., "Combustion Characteristics of Paraffin-Fueled Swirling Oxidizer-Flow-Type Hybrid Rocket Engines," $48^{\text {th }}$

AIAA/ASME/SAE/ASEE Joint Propulsion Conference, AIAA Paper No. 2012-3904, Atlanta, July 30 - Aug. 1, 2012.

17. Ozawa , K. and Shimada, T., "Linear Combustion Stability Analysis of Uni-directional Vortex Injection Hybrid Rocket Engines," 50th AIAA/ASME/SAE/ ASEE Joint Propulsion Conference, AIAA Paper No. 2014-3455,Cleveland, OH, July 28-30, 2014.

18. Beer, J.M. and Chigier, N.A., Combustion Aerodynamics, Applied Science Pub., London, 1972, p. 109-123.

19. Greatrix, D.R., Wozney, C. and Bockelt, M.W., "Alternative Thrust Modulation Techniques for Solid and Hybrid Rockets," Proceedings of the 65th International Astronautical Congress, Space Propulsion Symposium, Toronto, Sept 29 - Oct 3, 2014.

20. Brahmbhatt, V., Greatrix, D.R., Karpynczyk, J. and Trumpour, A.P., "Evaluation of a Laboratory-Scale Hybrid Rocket Engine's Performance," International Journal of Energetic Materials and Chemical Propulsion, Vol. 13, No. 2, 2014, pp. 123-139.

21. Summers, H. and Villarreal, J., "Small-Scale Hybrid Rocket Test Stand and Characterization of Swirl Injector," $49^{\text {th }}$ AIAA/ASME/SAE/ ASEE Joint Propulsion Conference, AIAA Paper No. 2013-3831, San Jose, July 14-17, 2013.

22. Bellomo, N., Barato, F., Faenza, M. and Lazzarin, M., "Numerical and Experimental Investigation on Vortex Injection in Hybrid Rocket Motors," $47^{\text {th }}$ AIAA/ASME/SAE/ ASEE Joint Propulsion Conference \& Exhibit, AIAA Paper No. 2011-5675, San Diego, July 31- August 3, 2011.

23. Wilkinson, R., Hart, K. and Day, R., "Proof-of-Concept Testing of Sustained Vortex-Flow Configuration for Hybrid Rocket Motors," $46^{\text {th }}$ AIAA/ASME/SAE/ ASEE Joint Propulsion Conference \& Exhibit, AIAA Paper No. 2010-6782, Nashville, July 25-28, 2013. 
24. Funami, Y. and Shimada, T., "Combine Analysis of Reactive Flow and Heat Transfer for Hybrid Rocket Design Engineering," 50th AIAA/ASME/SAE/ ASEE Joint Propulsion

Conference, AIAA Paper No. 2014-3456, Cleveland, OH, July 28-30, 2014. 